The Astronomical JournaL, 118:1577-1608, 1999 October

(C) 1999. The American Astronomical Society. All rights reserved. Printed in U.S.A.

\title{
THE INTERACTING GALAXIES NGC 5394/5395: A POST-OCULAR GALAXY AND ITS RING/SPIRAL COMPANION
}

\author{
Michele Kaufman, ${ }^{1}$ Elias Brinks, ${ }^{2}$ Bruce G. Elmegreen, ${ }^{3}$ Debra Meloy Elmegreen, ${ }^{4}$ Mario Klarić, ${ }^{5}$ \\ Curtis Struck, ${ }^{6}$ Magnus Thomasson, ${ }^{7}$ and Stuart Vogel ${ }^{8}$ \\ Received 1999 January 20; accepted 1999 June 8
}

\begin{abstract}
$\mathrm{H}$ I, radio continuum, Fabry-Perot $\mathrm{H} \alpha$, and ${ }^{12} \mathrm{CO} J=1 \rightarrow 0$ observations and broadband optical and near infrared images are presented of the interacting spiral galaxies NGC 5395 and NGC 5394. Kinematically, there are three important, separate components to the $\mathrm{H}$ I gas associated with this galaxy pair: (1) the main disk of NGC 5395, (2) a long, northern tidal arm of NGC 5395 distinct in velocity from its main disk, and (3) the disk of NGC 5394. The H I northern tidal arm of NGC 5395 has a line of-sight velocity as much as $75-100 \mathrm{~km} \mathrm{~s}^{-1}$ greater than the main disk of NGC 5395 at the same projected location and thus is not in the same plane as the disk. The velocity field of the disk of NGC 5395 is asymmetric and distorted by large-scale and small-scale noncircular motions. In NGC 5395, the encounter appears to be exciting $m=1$ and $m=0$ modes in what had been a two-armed spiral. The dominant spiral arm of NGC 5395 forms a large ring or pseudo-ring of $\mathrm{H} \alpha$, radio continuum, and $\mathrm{H}$ I emission, somewhat off center with respect to the nucleus. The H I trough in the center of NGC 5395 is not filled in by molecular gas. The $\mathrm{H} \alpha$ velocity contours exhibit an organized pattern of kinks in crossing the ring and also show streaming motions in a large stellar caustic feature. The eastern side of the ring is brighter in radio continuum and $\mathrm{H} \alpha$; the western side is brighter in $\mathrm{H}$ I and contains massive $\left(10^{8} M_{\odot}\right) \mathrm{H} \mathrm{I}$ clouds not associated with the most luminous H II regions. The smaller galaxy NGC 5394 is in an immediate post-ocular phase, with a central starburst, an intrinsically oval disk, two long, fairly symmetric, open tidal arms with high arm-interarm contrast, and very bright inner spiral arms, disjoint from the outer tidal arms. Most of the gas in NGC 5394 is in molecular form and concentrated within $3.8 \mathrm{kpc}$ of the center, so is suitable for fueling the starburst. Despite the presence of H I gas, two of the three optically bright inner spiral arms of NGC 5394 show no evidence of ongoing star formation.

A galaxy encounter simulation reproduces some of the main features of this system with a collision that is prograde relative to NGC 5394 and retrograde at a high tilt angle relative to NGC 5395. The model finds that the inner spiral structure of NGC 5394 developed from an eye-shaped ("ocular") structure at slightly earlier times. NGC 5394 and the two ocular galaxies IC 2163 and NGC 2535, studied earlier, form an evolutionary sequence of structures resulting from prograde encounters and thus confirm the generic models of such collisions. The agreement between the model for NGC 5394/95 and the ring/ spiral structures seen in NGC 5395 extends our understanding of collisional ring galaxies.
\end{abstract}

Key words: galaxies: individual (NGC 5394, NGC 5395) - galaxies: interactions galaxies: kinematics and dynamics - galaxies: spiral

${ }^{1}$ Department of Physics and Department of Astronomy, Ohio State University, 174 West 18th Avenue, Columbus, OH 43210; rallis@mps.ohio-state.edu.

${ }^{2}$ Departamento de Astronomía, Universidad de Guanajuato, Apdo. Postal 144, Guanajuato, GTO 36000, Mexico; ebrinks@astro.ugto.mx.

${ }^{3}$ IBM Research Division, T. J. Watson Research Center, P.O. Box 218, Yorktown Heights, NY 10598; bge@ watson.ibm.com.

${ }^{4}$ Vassar College Observatory, Poughkeepsie, NY 12601; Visiting Astronomer, Kitt Peak National Observatory, National Optical Astronomy Observatories, which is operated by the Association of Universities for Research in Astronomy, Inc., under cooperative agreement with the National Science Foundation; elmegreen@vassar.edu.

${ }^{5}$ Columbia, SC 29206; mariok@cyis.com.

${ }^{6}$ Department of Physics and Astronomy, Iowa State University, Ames, IA 50010; curt@iastate.edu.

7 Onsala Space Observatory, S-439 92 Onsala, Sweden; magnus@oso.chalmers.SE.

${ }^{8}$ Department of Astronomy, University of Maryland, College Park, MD 20742; vogel@astro.umd.edu.

\section{INTRODUCTION}

The interacting galaxies NGC 5394/95 (Arp 84) are one of five nearby galaxy pairs that we have observed with the $\mathrm{VLA}^{9}$ and other telescopes to study the structures and motions that result from a recent, close, nonmerging encounter. The results presented here on the tidal features of NGC 5394/95 complement our previous detailed studies of two other pairs in the set: IC 2163/NGC 2207 (Elmegreen et al. 1995a; Elmegreen et al. 1995b) and NGC 2535/36 (Kaufman et al. 1997; Klarić 1993). NGC 5394 resembles the galaxies IC 2163 and NGC 2535 in having

\footnotetext{
${ }^{9}$ The National Radio Astronomy Observatory is a facility of the National Science Foundation operated under cooperative agreement by Associated Universities, Inc.
} 
two long, symmetric, open tidal arms. Numerical simulations indicate that this type of tidal arm structure is produced by close, prograde, approximately in-plane encounters (see, for example, Toomre \& Toomre 1972; Elmegreen et al. 1991; Howard et al. 1993; Sundin 1993). IC 2163 and NGC 2535 both contain a central eye-shaped ("ocular") structure. In prograde encounter simulations, the eye-shaped structure is an early phase that lasts for only a short time. NGC 5394 appears to be in a stage of post-encounter evolution a little past the transient ocular stage. Comparing the tidal features of NGC 5394, IC 2163, and NGC 2535 allows us to see how the structures resulting from prograde, inplane encounters evolve.

Figure 1 displays the $r$-band image of the system from the Chengalur-Nordgren galaxy survey (Nordgren et al. 1997) with the two galaxies labelled. This galaxy pair is known to have several peculiar features. The smaller galaxy, NGC 5394, has inner spiral arms (see Fig. 1) described by
Arp (1969) and Wray (1988) as unusual, mostly smooth, high surface brightness arcs, near its sharp, very bright nucleus and two long, fairly symmetric, open tidal arms. The nucleus of NGC 5394 is a strong radio continuum source in the FIRST survey (Becker, White, \& Helfand 1995), and its optical absorption-line and emission-line spectra indicate a starburst with age $\approx 10^{7}$ years (Arp 1969; Keel et al. 1985). According to Arp, the western side of the larger galaxy, NGC 5395, is displaced outward and greatly widened. Sharp \& Keel (1985) find that the H I velocity width of NGC 5395 in single-dish profiles is $50 \%-100 \%$ greater than expected for its luminosity. They suggest a possible nonplanar distortion of the western arm and also note the stellar feature that we label "caustic 1 " in Figure 1 and they call "the ring between the arms."

To study the tidal features of NGC 5394/95 in more detail, we present $\mathrm{H}$ I and radio continuum observations taken at the VLA with the C and D configurations, $\mathrm{H} \alpha$

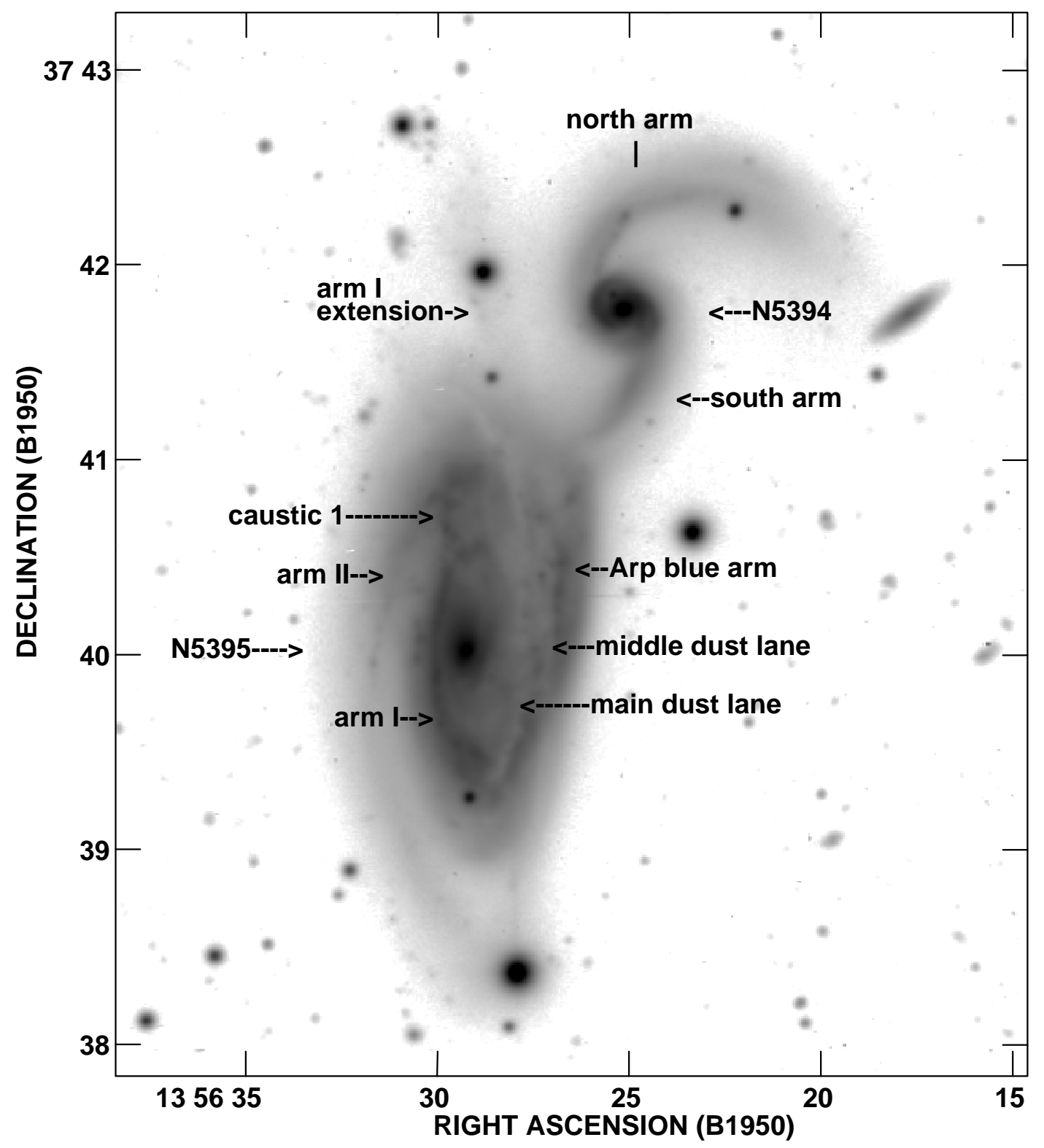

FIG. 1. $-r$-band image from the Chengalur-Nordgren galaxy survey with PSF $=2 " .6$ on a logarithmic intensity scale. The main disk of NGC 5394 shows spiral structure; outside of it, one can see two symmetrical tidal arms. On the western side of NGC 5395, arm I appears composed of several components that differ in curvature from one another, and part of this arm extends far to the north of the main disk. 
observations taken with the Maryland-Caltech FabryPerot camera, optical and near-infrared images taken with the $2.1 \mathrm{~m}$ telescope at KPNO, with the Fick Observatory $0.6 \mathrm{~m}$ telescope, and with the Burrell-Schmidt telescope, and ${ }^{12} \mathrm{CO}$ data from Onsala Space Observatory. ${ }^{10}$

The distance to NGC 5394/95 is assumed to be $47 \mathrm{Mpc}$, based on $H_{0}=75 \mathrm{~km} \mathrm{~s}^{-1} \mathrm{Mpc}^{-1}$; then $1^{\prime \prime}=230 \mathrm{pc}$. Table 1 lists the basic galaxy properties from the Third Reference Catalogue (de Vaucouleurs et al. 1991) and the NASA Extragalactic Database (NED). ${ }^{11}$ As Sharp \& Keel (1985) point out, the determination of the systemic velocities of these two galaxies has had a checkered history. All of the velocities given in this paper are heliocentric and use the optical definition for the nonrelativistic Doppler shift.

Section 2 describes the observations and the data reduction. Section 3 presents the morphology of the galaxy pair in the optical, near-infrared, $\mathrm{H} \alpha$, and radio continuum; $\S 4$, the surface photometry in $R$ and $J$ bands; $\S 5$, the $\mathrm{H} \alpha$ velocity field; $\S 6$, the $\mathrm{H}$ I properties and the separate kinematic components; and $\S 7$, the ${ }^{12} \mathrm{CO} J=1 \rightarrow 0$ detections. Section 8 discusses the results and the constraints on the orbits of the two galaxies. Section 9 presents a galaxy encounter simulation that reproduces some of the main optical and $\mathrm{H}$ I properties of this system. Section $10 \mathrm{com}$ pares NGC 5394 with two ocular galaxies studied earlier, and $\S 11$ contains our conclusions.

\section{OBSERVATIONS AND DATA REDUCTION}

\subsection{Optical and Near-Infrared CCD Images}

At the Fick Observatory we took $R$-band and $\mathrm{H} \alpha \mathrm{CCD}$ images of NGC 5394/95 on 1996 June 23 and 1996 July 11 with a total exposure of $600 \mathrm{~s}$ in $R$-band and $3600 \mathrm{~s}$ in $\mathrm{H} \alpha$. The field of view is about $15^{\prime} \times 15^{\prime}$, and the pixel size is $1^{\prime \prime} .3$. The images were transformed to right ascension and declination coordinates so that we could later bring our various images to the same coordinate grid. The combined $R$-band image has a point-spread function (PSF) of $4.2 \times 3$ ". 8 , and the combined $\mathrm{H} \alpha$ image has a PSF of 5".2 $\times$ 4".5.

\footnotetext{
${ }^{10}$ Onsala Space Observatory at Chalmers University of Technology is the Swedish National Facility for Radio Astronomy.

${ }^{11}$ The NASA/IPAC Extragalactic Database (NED) is operated by the Jet Propulsion Laboratory, California Institute of Technology, under contract with the National Aeronautics and Space Administration.
}

The plate solutions for these images and for the $\mathrm{H} \alpha$ Fabry-Perot data discussed below were based on secondary standard stars, using the Guide Star Catalog and the Digitized Sky Survey image, and have standard deviations $\leq 0$ ".5. As a check, we find that the position of the NGC 5394 nucleus on the $R$-band and $\mathrm{H} \alpha$ images differs from the position of the nuclear radio continuum source in the FIRST survey (Becker et al. 1995) by $\leq 0$ ".6 in right ascension and $\leq 1{ }^{\prime \prime} .0$ in declination. (The stated uncertainty of the FIRST survey position is 0 ".4).

An I-band CCD image of NGC 5394/95 was taken with the Burrell-Schmidt telescope in 1997 February. The exposure time was $1000 \mathrm{~s}$, and the pixel size is $2^{\prime \prime}$. Because of poor seeing during that observing run, we use this image only (1) to obtain the ratio of the $I$-band luminosities of the galaxies in order to estimate their mass ratio and (2) to confirm the arm-interarm contrast measured in the $R$ band for the tidal arms of NGC 5394.

We took near-infrared $J$ - and $K$-band images of NGC 5394 and the central portion of NGC 5395 with the $2.1 \mathrm{~m}$ telescope and the cryogenic optic bench detector at Kitt Peak on 1996 April 31-May 5. The images were skysubtracted and flat-fielded with a global flat made from the skies of the whole observing run. The exposures consisted of on-galaxy images interleaved with off-galaxy images of equal exposure times, from which local skies were made. The $J$-band image of NGC 5394 has a total exposure of 900 s, the $K$-band image of NGC 5394, a total exposure of 450 s, and the total field of view is approximately $60^{\prime \prime} \times 60^{\prime \prime}$. The $J$-band image of NGC 5395 has a total exposure of $900 \mathrm{~s}$ and a total field of view of approximately $60^{\prime \prime} \times 50^{\prime \prime}$. The pixel size is 0.2 , and the average seeing was $1^{\prime \prime}$.

\subsection{Fabry-Perot $\mathrm{H} \alpha$ Observations}

Using the Maryland-Caltech wide-field imaging FabryPerot interferometer, $\mathrm{H} \alpha$ observations were made of NGC $5394 / 95$ at the Palomar $1.5 \mathrm{~m}$ telescope ${ }^{12}$ on 1990 June 3. The instrument images a $16^{\prime}$ field of view onto a CCD with 0 ".94 pixels. Seeing limited the angular resolution to about 2".4. The Fabry-Perot etalon has a free-spectral range of

\footnotetext{
12 The Palomar 60 inch telescope is operated jointly by the California Institute of Technology and the Carnegie Institution of Washington.
}

TABLE 1

BASIC DATA ON NGC 5394/5395

\begin{tabular}{|c|c|c|}
\hline Characteristic & NGC 5394 & NGC 5395 \\
\hline Morphological type & $\mathrm{SB}(\mathrm{s}) \mathrm{b}$ pec & $\mathrm{SA}(\mathrm{s}) \mathrm{b}$ pec \\
\hline Right ascension (1950.0). & $13^{\mathrm{h}} 56^{\mathrm{m}} 25^{\mathrm{s}} .10$ & $13^{\mathrm{h}} 56^{\mathrm{m}} 29^{\mathrm{s}} .23$ \\
\hline Declination $(1950.0) \ldots \ldots \ldots \ldots$ & $+37^{\circ} 41^{\prime} 45^{\prime \prime} .7$ & $+37^{\circ} 40^{\prime} 00^{\prime \prime} .7$ \\
\hline Isophotal major radius $R_{25} \ldots \ldots \ldots \ldots \ldots$ & 0.85 & 1.45 \\
\hline 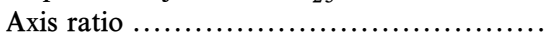 & 1.7 & 2.3 \\
\hline$B$ magnitude $B_{T} \ldots \ldots \ldots \ldots \ldots \ldots \ldots \ldots \ldots \ldots \ldots \ldots \ldots \ldots \ldots$ & 13.70 & 12.10 \\
\hline Corrected $B^{0}{ }_{T} \ldots \ldots$ & 13.28 & 12.01 \\
\hline$(B-V)_{T}(\mathrm{mag}) \ldots \ldots \ldots \ldots \ldots . .$. & 0.69 & 0.73 \\
\hline Optical heliocentric $v$ (nucleus) $\left(\mathrm{km} \mathrm{s}^{-1}\right) \ldots \ldots$ & 3451 & 3493 \\
\hline 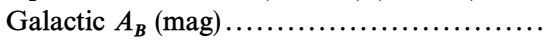 & \multicolumn{2}{|c|}{0.00} \\
\hline 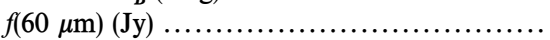 & \multicolumn{2}{|c|}{9.07} \\
\hline 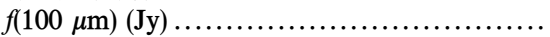 & \multicolumn{2}{|c|}{21.51} \\
\hline 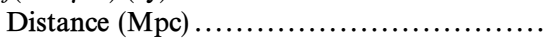 & \multicolumn{2}{|c|}{47} \\
\hline
\end{tabular}

${ }^{a}$ Axis ratio of NGC 5395 from Kodeira et al. (1990); position of NGC 5395 nucleus from this paper; distance based on $H_{0}=75 \mathrm{~km} \mathrm{~s}^{-1} \mathrm{Mpc}^{-1}$; rest of the data from de Vaucouleurs et al. (1991) and the NASA Extragalactic Database. 
about $500 \mathrm{~km} \mathrm{~s}^{-1}$ and a spectral resolution of $25 \mathrm{~km} \mathrm{~s}^{-1}$ at $\mathrm{H} \alpha$. A $20 \AA\left(1000 \mathrm{~km} \mathrm{~s}^{-1}\right)$ FWHM blocking filter centered near $6640 \AA$ transmits $\mathrm{H} \alpha$ at the galaxy redshift. Because the etalon free spectral range is smaller than the range of velocities present in NGC 5394/95, at some velocities there is a $500 \mathrm{~km} \mathrm{~s}^{-1}$ ambiguity in the $\mathrm{H} \alpha$ velocity; however, we resolve the ambiguity using the $\mathrm{H}$ I velocities. Due to the nonrectangular shape of the filter transmission profile, some $[\mathrm{N} \mathrm{II}]$ is also transmitted. The blocking filter attenuates the $[\mathrm{N} \mathrm{II}] / \mathrm{H} \alpha$ ratio by a factor of $0.1-0.5$, depending on velocity, with the result that the attenuated [N II] line is generally weak compared to $\mathrm{H} \alpha$. We acquired 47 frames, stepping at approximately $12 \mathrm{~km} \mathrm{~s}^{-1}$, to cover the free-spectral range plus a small additional amount for overlap; each exposure was $300 \mathrm{~s}$. Cosmic rays were removed and the data flatfielded, and then the frames were registered and gridded onto a cube with 0 ".94 pixels and monochromatic planes spaced by $12 \mathrm{~km} \mathrm{~s}^{-1}$. A cube of size $201 \times 320 \times 52$ pixels (R.A. $\times$ decl. $\times$ velocity) was extracted, covering a velocity range of 3216 to $3835 \mathrm{~km} \mathrm{~s}^{-1}$.

Neon lamp exposures were used to establish the spectral calibration. Unfortunately, during the epoch of the observations the setup allowed transmission of a large number of neon lines, which reduces the accuracy of the velocity calibration. Night-skylines were not bright enough in the observed spectral region to reduce this uncertainty. As a result, the absolute velocity calibration is uncertain to about $50 \mathrm{~km} \mathrm{~s}^{-1}$. Also, there is a channel-width uncertainty that increases monotonically with angular separation along the radial direction with respect to the etalon optical axis (which is approximately the east-west direction for these observations). Specifically, over large east-west displacements, errors in velocity differences may get as large as 50 $\mathrm{km} \mathrm{s}^{-1}$; but over displacements $\lesssim 10^{\prime \prime}-20^{\prime \prime}$, errors in relative velocities are small. The implication is that the $\mathrm{H} \alpha$ data are of limited use for large-scale kinematics, such as rotation curve analysis. However, for the determination of kinematic structure on scales less than $10^{\prime \prime}$ to $20^{\prime \prime}$, such as revealed by kinks in isovelocity contours, the errors in relative velocities are negligible.

The velocity field images displayed in $\S 5$ were constructed by fitting a Gaussian to the line profile at each position. In addition, for comparison with the $\mathrm{H}$ I velocity field, the $\mathrm{H} \alpha$ cube was convolved to the same resolution as the $\mathrm{H}$ I cube, and moment maps, i.e., intensity-weighted velocity fields and total intensity maps, were made. The $\mathrm{H} \alpha$ velocity field is used only to search for streaming motions over small spatial scales (kinks in the isovelocity contours).

The CCD $\mathrm{H} \alpha$ surface brightness image from the Fick Observatory served as a check on various features which can be seen in the total intensity Fabry-Perot image.

\section{3. $\mathrm{H} \mathrm{I}$}

We observed NGC 5394/95 in H I at the VLA for $5.2 \mathrm{hr}$ (on the target) in C configuration on 1993 July 11 and for 1 $\mathrm{hr}$ in D configuration on 1993 October 30. In both cases, $1504+377$ served as the phase calibrator, and 3C 286 as the flux standard and bandpass calibrator. We assumed a flux density of $14.9 \mathrm{Jy}$ for 3C 286 on the Baars et al. (1977) scale. The data were taken in the 2AD correlator mode with two passbands, each $3.125 \mathrm{MHz}$ wide and containing 64 channels, and with a 16-channel overlap between the two bands. The two passbands were centered at 3767.940 and 3261.599 $\mathrm{km} \mathrm{s}^{-1}$, respectively. After omitting the high-noise channels at each end, the combination covers heliocentric velocities $2988-4043 \mathrm{~km} \mathrm{~s}^{-1}$ with a channel width of 48.828 $\mathrm{kHz}=10.57 \mathrm{~km} \mathrm{~s}^{-1}$ after on-line Hanning smoothing. H I emission is present for heliocentric velocities $3125-3810 \mathrm{~km}$ $\mathrm{s}^{-1}$; the line-free channels were averaged to create continuum images.

The AIPS software package was used for the data reduction. The uv data from each of the VLA configurations and each of the two IFs were calibrated separately. Some solar interference present in the D configuration data was removed by flagging emission in the uv data above a certain flux limit after first subtracting the stronger continuum sources. Then for each uv data set separately, we generated "dirty" data cubes of line plus continuum emission and subtracted the continuum emission from each channel. The resulting H I maps were cleaned and found satisfactory on inspection. Then the uv data sets from the $\mathrm{C}$ and $\mathrm{D}$ configurations were combined, the above mapping and cleaning procedure repeated, first with natural weighting and then with uniform weighting, and the cubes from the two IFs were appropriately merged. We selected areas of genuine $\mathrm{H}$ I emission by the following procedure. The naturally weighted cube was convolved to $30^{\prime \prime}$ resolution and clipped at $1.3 \mathrm{mJy}$ beam $^{-1}$, which is twice the rms noise. Regions of $\mathrm{H} \mathrm{I}$ emission were retained in this cube only if the feature appeared in at least two adjacent velocity channels. The resulting cube was applied as a blanking mask to the higher resolution cubes to produce two final full-resolution subcubes: one made with natural weighting and spatial resolution $=18.4 \times 17^{\prime \prime} .7(\mathrm{FWHM})$ and referred to hereafter as the low-resolution cube; the other with uniform weighting and spatial resolution $=11^{\prime \prime} 4$ (FWHM) and referred to hereafter as the high-resolution cube. Moment maps made from these cubes will be denoted low resolution and high resolution, respectively. Additionally, we blanked regions in the first and second moment images where the $\mathrm{H}$ I surface brightness was low in the zeroth moment image. Both final subcubes have pixel size $=3^{\prime \prime}$, channel width $=10.6 \mathrm{~km} \mathrm{~s}^{-1}$, and dimensions $=$ $143 \times 166 \times 72$ pixels (R.A. $\times$ decl. $\times$ velocity).

We measure a total line flux for the galaxy pair of $30 \mathrm{Jy}$ $\mathrm{km} \mathrm{s}^{-1}$, corresponding to an $\mathrm{H}$ I mass of $1.6 \times 10^{10}\left(75 / \mathrm{H}_{0}\right)^{2}$ $M_{\odot}$. This is consistent with the single-dish observations by Krumm \& Salpeter (1980), who obtained $32 \mathrm{Jy} \mathrm{km} \mathrm{s}^{-1}$. In $\S 6$ below, we use kinematic information to separate the $\mathrm{H}$ I contributions of the two galaxies. In fact, as we shall see, there are three important, separate kinematic features in the H I data.

Cleaned images of the $\lambda 20 \mathrm{~cm}$ radio continuum emission were made from the averaged, line-free uv data. We measure a total $\lambda 20 \mathrm{~cm}$ continuum flux density $S_{v}(20)$ of 100 $\mathrm{mJy}$ for the galaxy pair, consistent with the previous VLA measurement of $S_{v}(20)=94 \mathrm{mJy}$ by Condon et al. (1990).

Table 2 gives a summary of the $\mathrm{H}$ I and radio continuum map parameters.

\section{4. $\mathrm{CO}$}

Single-dish ${ }^{12} \mathrm{CO} J=1 \rightarrow 0$ observations were made with the $20 \mathrm{~m}$ telescope at Onsala Space Observatory on four occasions: 1994 April 14-17, 1994 May 14-16, 1995 January 18, and 1995 March 28-April 3. The front end was a cooled SIS receiver, and the back end consisted of a filter bank with 512 channels and a frequency resolution of 1 $\mathrm{MHz}$ (corresponding to a velocity resolution of $2.6 \mathrm{~km} \mathrm{~s}^{-1}$ 
TABLE 2

H i and Radio ContinuUm MaPs From Combined C AND D Configuration Data

\begin{tabular}{|c|c|c|}
\hline Parameter & $\begin{array}{l}\text { Natural Weight } \\
\text { (“Low Resolution") }\end{array}$ & $\begin{array}{l}\text { Uniform Weight } \\
\text { ("High Resolution") }\end{array}$ \\
\hline \multicolumn{3}{|l|}{ H I images: } \\
\hline Synthesized beam (FWHM, P.A.).. & $18^{\prime \prime} .4 \times 17^{\prime \prime} .7,90^{\circ}$ & $11^{\prime \prime} .4 \times 11^{\prime \prime} .4,0^{\circ}$ \\
\hline Conversion factor $\left(T_{b} / \mathrm{S}\right)\left(\mathrm{K} / \mathrm{mJy}\right.$ beam $\left.^{-1}\right) \ldots \ldots$ & 1.90 & 4.77 \\
\hline rms noise per channel $\left(\mathrm{mJy}\right.$ beam $\left.{ }^{-1}\right) \ldots \ldots \ldots \ldots . .$. & 0.46 & 0.78 \\
\hline $\begin{array}{l}\text { Column density in atoms } \mathrm{cm}^{-2} \text { equivalent to } \\
100 \mathrm{Jy} \text { beam }^{-1} \mathrm{~m} \mathrm{~s}^{-1} \ldots \ldots \ldots \ldots \ldots \ldots \ldots \ldots\end{array}$ & $3.4 \times 10^{20}$ & $8.5 \times 10^{20}$ \\
\hline \multicolumn{3}{|l|}{ Radio continuum images: } \\
\hline Synthesized beam (FWHM, P.A.)............... & $18.4 \times 17^{\prime \prime} .7,90^{\circ}$ & $11^{\prime \prime} 4 \times 11^{\prime \prime} 4,0^{\circ}$ \\
\hline Conversion factor $\left(T_{b} / S\right)\left(\mathrm{K} /\right.$ mJy beam $\left.^{-1}\right) \ldots \ldots$ & 1.90 & 4.77 \\
\hline rms noise $\left(\mathrm{mJy}\right.$ beam $\left.{ }^{-1}\right) \ldots \ldots \ldots \ldots \ldots \ldots \ldots \ldots$ & 0.090 & 0.14 \\
\hline
\end{tabular}

at $115 \mathrm{GHz}$ ). The beam size (HPBW) was $33^{\prime \prime}$, and the main beam efficiency 0.5 . We used the beam switch observing method with a beam throw of $12^{\prime}$, and the standard chopper wheel method for calibration. The system temperature (including atmosphere) was typically between $500 \mathrm{~K}$ and $700 \mathrm{~K}$, occasionally somewhat larger or smaller. We observed eight positions in NGC 5395 and five positions in NGC 5394 with a spacing of $33^{\prime \prime}$, and an additional four positions in NGC 5394, 16".5 north, south, east, and west of the center position. The integration time per position varied between $0.9 \mathrm{hr}$ and $2.7 \mathrm{hr}$, with an average of $1.5 \mathrm{hr}$, for the 13 positions with $33^{\prime \prime}$ spacing. For the four extra positions in NGC 5394, the average integration time was $0.6 \mathrm{hr}$. The total observation time was $23 \mathrm{hr}$ (all numbers given as time on-source). Pointing was checked regularly using $\mathrm{SiO}$ masers (e.g., TX Cam, R Leo, T Cep, and R Cas) as reference sources. The pointing errors were estimated to be less than one tenth of the beam size, except for the four extra positions in NGC 5394, where we had pointing problems. The raw data were reduced using the Data Reduction Package developed by Michael Olberg at Onsala Space Observa- tory. The intensities from the chopper wheel calibration, given on the $T_{A}^{*}$ temperature scale, were divided by the main-beam efficiency $\left(\eta_{\mathrm{mb}}=0.5\right)$ to get the main-beam temperatures $T_{\mathrm{mb}}$. The total spectra were Hanning filtered and then smoothed to a velocity resolution of $5.2 \mathrm{~km} \mathrm{~s}^{-1}$, resulting in a noise with a typical rms value of $0.017 \mathrm{~K}$ (on the $T_{\mathrm{mb}}$ temperature scale); the four positions 16 ".5 off the center in NGC 5394 have a somewhat higher noise level of about $0.026 \mathrm{~K}$.

\section{MORPHOLOGY}

\subsection{Optical and Near-Infrared 3.1.1. NGC 5395}

As Arp (1969) pointed out, the whole western side of NGC 5395 appears very wide and displaced outward relative to the nucleus (see Fig. 1 and the enlarged display of the Arp atlas plate in Sharp \& Keel 1985). Sharp \& Keel present convincing arguments that the western side of NGC 5395 is the near side. The orientations of the galaxies are described in Table 3. For the optical disk of NGC 5395 at

TABLE 3

ORIENTATION PARAMETERS AND SYSTEMIC Velocities

\begin{tabular}{|c|c|}
\hline Parameter & Value \\
\hline \multicolumn{2}{|l|}{ NGC 5395: } \\
\hline \multicolumn{2}{|l|}{ Major axis position angle of disk (deg): } \\
\hline Photometric (25th mag. isophote) ..... & $173 \pm 2$ \\
\hline Photometric on ring .................. & $173 \pm 2$ \\
\hline H i kinematic ....................... & $170 \pm 2$ \\
\hline \multicolumn{2}{|l|}{ Minor axis position angle of disk (deg): } \\
\hline H I kinematic ......................... & $83 \pm 2$ \\
\hline Preferred position angle of projection line of nodes. & $173 \pm 2$ \\
\hline Disk inclination $(\mathrm{deg})$.................. & $65 \pm 2$ \\
\hline \multicolumn{2}{|l|}{ Systemic velocity $\left(\mathrm{km} \mathrm{s}^{-1}\right)$ : } \\
\hline H I velocity field. & $3450 \pm 10$ \\
\hline $\mathrm{H}$ I at nucleus ... & $3496 \pm 17$ \\
\hline Adopted value...... & $3450 \pm 10$ \\
\hline \multicolumn{2}{|l|}{ NGC 5394: } \\
\hline \multicolumn{2}{|l|}{ Minor axis position angle of disk (deg): } \\
\hline H I kinematic ............. & $90 \pm 2$ \\
\hline Preferred position angle of projection line of nodes $(\mathrm{deg}) \ldots \ldots$ & $0 \pm 2$ \\
\hline 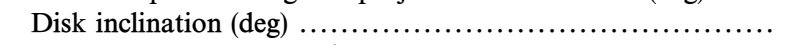 & $9 \pm 2$ \\
\hline \multicolumn{2}{|l|}{ Systemic velocity $\left(\mathrm{km} \mathrm{s}^{-1}\right)$ : } \\
\hline H I velocity field ...... & $3470 \pm 5$ \\
\hline $\mathrm{H}$ I at nucleus. & $3464 \pm 3$ \\
\hline Adopted value ...... & $3465 \pm 5$ \\
\hline
\end{tabular}


the 25th magnitude isophote, Kodeira, Okamura, \& Ichikawa (1990) find the position angle of the major axis is $173^{\circ}$ and the axis ratio is 0.43 . If the galaxy is an intrinsically circular, thin disk (not necessarily a good assumption given the outward displacement of the western arm), then the inclination $i=65^{\circ}$ (where $i=0^{\circ}$ for a face-on galaxy).

The two spiral arms of NGC 5395 are labelled "arm I" and "arm II" in Figure 1. Arm I is much brighter than arm II; this suggests that the encounter is exciting an $m=1$ mode in what had been a two-armed spiral. On the western side of NGC 5395, arm I appears composed of several components differing in curvature from one another. These are marked in Figure 1: the main dust lane (a long prominent dust lane that runs along the inside edge of the arm and curves toward the north-northeast at its northern end), the middle dust lane (a somewhat shorter dust lane along the middle of the arm), and the Arp blue arm (see the color picture in Wray 1988), which runs along the outside edge and points due north toward the southern tidal arm of NGC 5394. The differences in pitch angle led Sharp \& Keel (1985) to conjecture that part of arm I on the western side of NGC 5395 is in a different plane. Because of our viewing angle and the large inclination of the disk, the sky-plane projection can have a significant effect on the apparent location of an arm not in the disk plane, e.g., as a result of perpendicular forcing in the collision; relative to the disk, such an arm would appear displaced to the east if in front (or to the west if behind) compared with where it would be seen in a face-on view. The middle portion of arm I on the western side of NGC 5395 has a faint extension (marked "arm I extension" in Fig. 1) far north of the galaxy and east of the companion. In $\S 6.2$ below, we find a prominent $\mathrm{H} \mathrm{I}$ arm closely parallel to this faint optical extension. The highresolution $J$-band image of the center of NGC 5395 is not displayed, as it reveals no interesting structures.

In Figure 2, contours from our Fick Observatory $R$-band image of the system are overlaid on a gray-scale display of a Fabry-Perot $\mathrm{H} \alpha$ surface brightness image. The $\mathrm{H} \alpha$ image has composite resolution: regions of high surface brightness are displayed at full spatial resolution (2".4 FWHM), regions of moderate brightness were smoothed with a 3 pixel FWHM Gaussian, and faint regions were smoothed with an 8 pixel FWHM Gaussian. Fabry-Perot $\mathrm{H} \alpha$ images constructed in this fashion from the cube will be referred to hereafter as composite-resolution $\mathrm{H} \alpha$ images. There is an outer string of $\mathrm{H}$ in regions along the Arp blue arm of NGC 5395.

On the northeast side of NGC 5395, there is a $20^{\prime \prime}(=4.6$ kpc) diameter stellar feature (labelled "caustic 1" in Fig. 1). It appears in the $\mathrm{H} \alpha$ and the $R$-band images (see Fig. 2), in the $B$-band image in Sharp \& Keel (1985), and in the $V$-band image in Kodeira et al. (1990). Thus, it is a stellar feature and a feature in the ionized gas. Because of the involvement of older stars, the most likely interpretation is that this structure is a caustic produced by temporary convergence of orbits as a result of the interaction (as suggested by the model in $\S 9$ below), rather than a superbubble. Caustic 1 will be discussed further in $\S 5$, when we describe the $\mathrm{H} \alpha$ velocity field, and in $\S 9$, where we present an encounter simulation.

\subsubsection{NGC 5394}

Figures 1 and 2 and show that NGC 5394 has two long, fairly symmetric, open tidal arms. Close, prograde, approx- imately in-plane encounters produce this type of tidal arm structure. As Arp (1969) pointed out, the bright part of the northern tidal arm appears to have a sharp string of knots along its inner edge. A thin dust lane (not easily visible in the figures shown here) abuts the convex side of this star formation ridge; in $R$-band the dust lane is $0.5 \mathrm{mag}$ fainter than the star formation ridge and 0.1 mag fainter than the rest of the arm. This convex position for the dust lane suggests that much of the northern tidal arm of NGC 5394 is outside of its corotation radius. At the end of the northern tidal arm, an edge-on galaxy is seen. No information is available about its redshift; it is not detected in our H I observations or in $\mathrm{H} \alpha$. From the sky-subtracted $I$-band image, we find that the luminosity of NGC 5394 is $0.25 \pm 0.006$ times the luminosity of NGC 5395 . Using the $r$-band image of Nordgren et al. (1997) because of its superior resolution, we find that the luminosity of the central starburst region (diameter $=5^{\prime \prime}$, see $\S 3.2$ below) is $15 \%$ of the total $r$-band luminosity of NGC 5394 . We therefore adopt a mass ratio for NGC 5394/95 of $0.23 \pm 0.02$. A mass ratio from "rotation curves" for these galaxies would be highly uncertain because of strong noncircular motions in the outer parts of the galaxies (see $\S 6$ below), because of the intrinsically distorted shape of NGC 5394 (see $\S \S 4,6.5$, and 9 below), which invalidates use of isophotes to derive its inclination, and because of the nearly face-on orientation of NGC 5394 (see $\S 6.5$ ). The $I$-band flux from the edge-on galaxy is $0.024 \pm 0.001$ times the flux from NGC 5395.

The central portion of the $J$-band image of NGC 5394 is displayed as an unsharp masked image in Figure 3. Because of the excellent seeing conditions, the two inner spiral arms on the western side of the nucleus and the inner spiral arm on the eastern side of the nucleus are resolved. These arms are described by Wray (1988) as extremely bright spiral arcs that are "mostly smooth and green."

In Figure 4, surface brightness contours from the fullresolution (2".4 FWHM), Fabry-Perot $\mathrm{H} \alpha$ image are overlaid on the $J$-band image in gray scale. The $J$-band spiral arm on the eastern side of the nucleus is not detected in $\mathrm{H} \alpha$ emission. This is unusual because high surface brightness in a spiral arm is almost always the result of ongoing star formation, but it is consistent with Wray's description that the arcs are mostly smooth. On the western side of the nucleus, the extended $\mathrm{H} \alpha$ emission is associated mainly with the $J$-band spiral arm that is closer to the nucleus. Sharp \& Keel (1985) similarly noted a possible asymmetry in the distribution of $\mathrm{H} \alpha$ emission from the center of NGC 5394. The $\mathrm{H} \alpha$ knots $30^{\prime \prime}$ north of the nucleus and $15^{\prime \prime}$ northnortheast of the nucleus are along the northern tidal arm (see Fig. 2). Notice that the northern tidal arm departs from the side of the $J$-band spiral arm at a large angle and not from the end of the $J$-band spiral arm. This is also evident in the $r$-band image of Figure 1. The $J$-band spiral arms are clearly inner spirals, not the rim of an ocular oval, and are disjoint from the tidal arms. The resolved blob $6^{\prime \prime}$ northeast of the nucleus in the $J$-band image is probably a background galaxy unrelated to this system.

\section{2. $\mathrm{H} \alpha$ and Radio Continuum}

Figure 5 overlays contours from the high-resolution (11".4 FWHM) $\lambda 20 \mathrm{~cm}$ radio continuum image on a gray-scale display of the composite-resolution $\mathrm{H} \alpha$ surface brightness image of the system. 


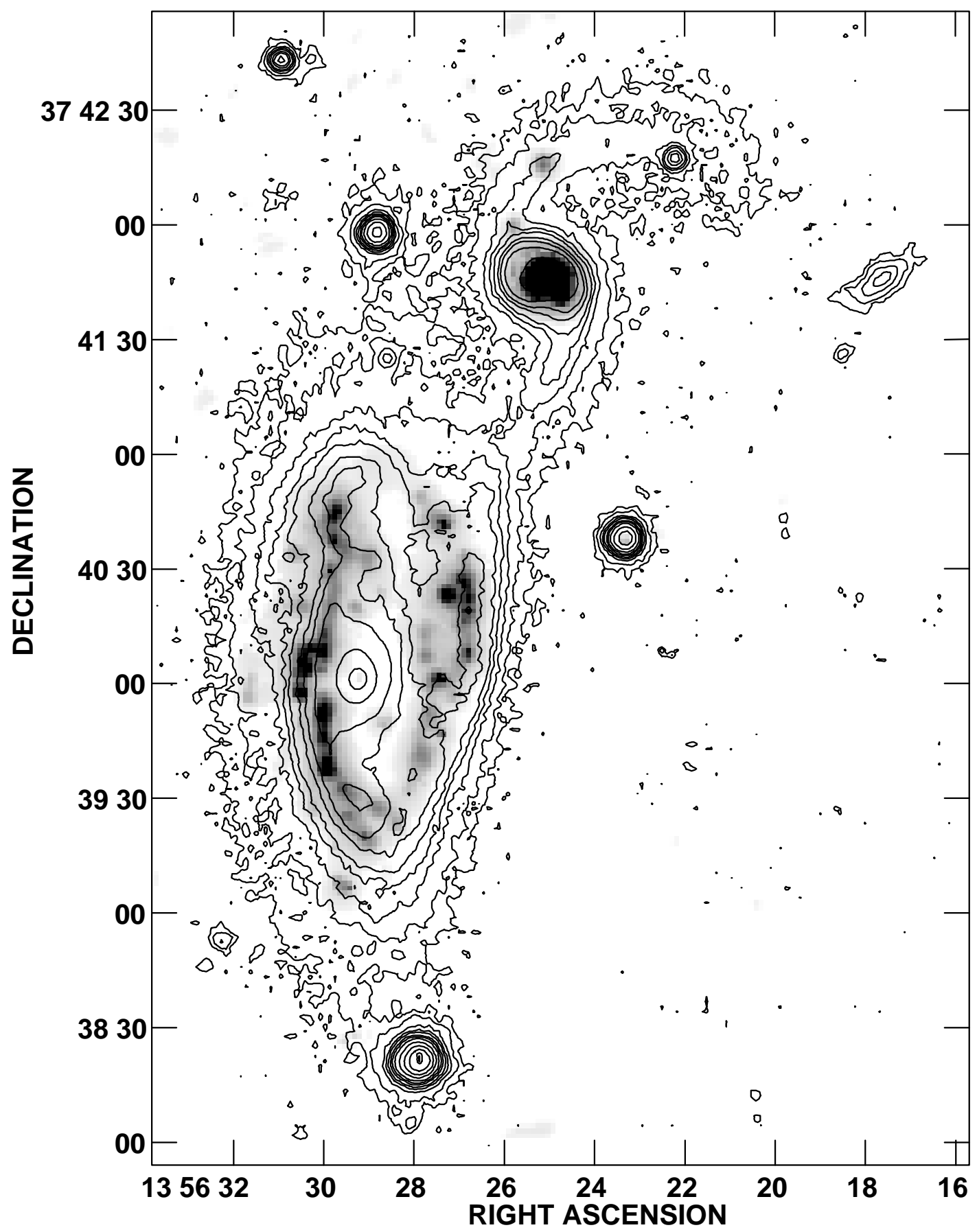

FIG. 2. - Gray-scale display of the composite-resolution $\mathrm{H} \alpha$ image of the galaxy pair with contours from the Fick Observatory $R$-band image overlaid

In NGC 5395, the $\mathrm{H} \alpha$ and radio continuum emission form a large ring or a pseudo-ring composed largely of arm I. We shall refer to this structure as the $\mathrm{H} \alpha$ and radio continuum ring or, for short, the ring. The ring interpretation of this feature is supported by the velocity data in $\S 5$ below. The presence of the ring suggests that the encounter may also involve an $m=0$ mode (see $\S 9$ below). With the following values for the projection parameters, the ring deprojects to a circle of radius $46^{\prime \prime}(=11 \mathrm{kpc})$ : ring center at $6^{\prime \prime}$ west, $10^{\prime \prime}$ north of the nucleus, major-axis position angle $=173^{\circ}$, and $i=67^{\circ}$. The position angle and inclination of the ring lie within $2^{\circ}$ of the values obtained for the optical disk at the 25 th magnitude isophote (see Table 3 ).
The eastern (anticompanion) side of the ring is brighter in the radio continuum and has a greater number of highly luminous $\mathrm{H}$ II regions; $60 \%$ of the radio continuum and $\mathrm{H} \alpha$ emission from the ring comes from its eastern half. On the high-resolution radio continuum image, the values of $S_{v}(20)$ from the northeast, southeast, northwest, and southwest quadrants of the ring are $17 \mathrm{mJy}, 14 \mathrm{mJy}, 13 \mathrm{mJy}$, and 7 mJy, respectively, so there is a notable deficiency in the southwest quadrant. On the low-resolution radio continuum image, the total $S_{v}(20)$ of NGC 5395 is $64 \mathrm{mJy}$. If M51 were at the distance of NGC 5395, it would have a total $S_{v}(20)$ of $63 \mathrm{mJy}$ (about the same as NGC 5395) and the most luminous $\mathrm{H}$ II region (CCM 72) in M51 would have an 


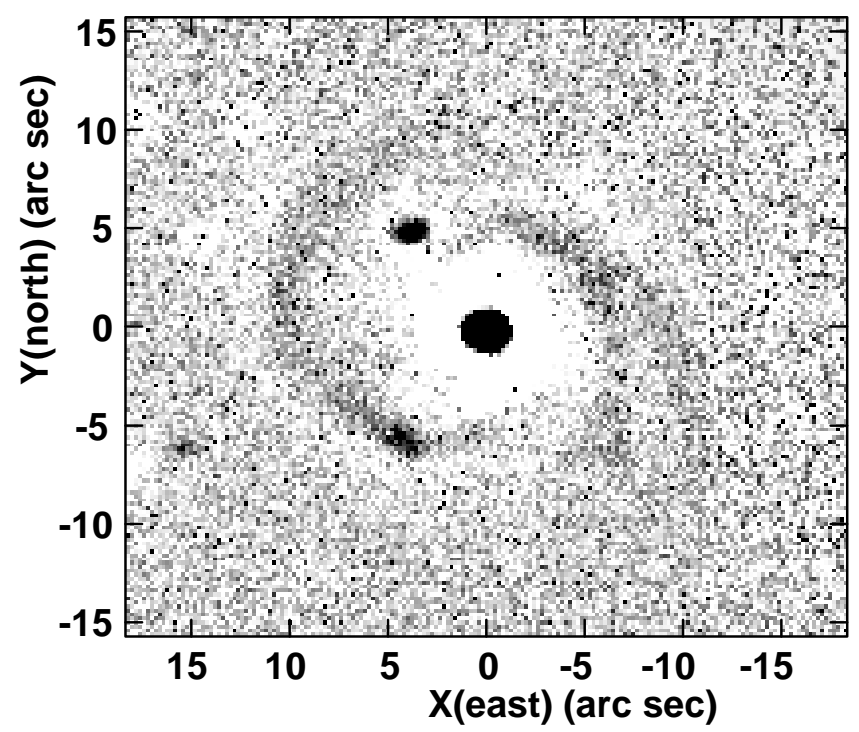

FIG. 3.-Unsharp masked $J$-band image of the central part of NGC 5394. This shows the inner spiral, which has a double arm on its western side.
$S_{v}(20)$ of $0.070 \mathrm{mJy}$ and a size of $2^{\prime \prime}$ (van der Hulst et al. 1988). The brightest $\lambda 20 \mathrm{~cm}$ emission from NGC 5395 occurs 3 " south of the southeast corner of caustic 1 . We find a peak radio brightness of $4.4 \mathrm{mJy}^{\text {beam }}{ }^{-1}$; this source is also listed in the FIRST survey with an integrated flux $S_{v}(20)$ of $9.6 \mathrm{mJy}$. The NGC 5395 nucleus is not prominent in $\mathrm{H} \alpha$ or in the radio continuum; Keel et al. (1985) measure its $H \alpha$ equivalent width as $1.5 \AA$ and classify the spectrum as old population plus LINER. In Figure 5, three $\mathrm{H} \alpha$ features in NGC 5395 are labelled: caustic 1 and two possible shells that will be discussed in $\S 5.1$. These are not seen as distinct structures in the radio continuum image (which has lower resolution).

The brightest $\mathrm{H} \alpha$ and radio continuum source in the system is the starburst nucleus of NGC 5394. Using a 4".7 aperture, Keel et al. measure its $\mathrm{H} \alpha$ equivalent width as 56.5 $\AA$ and its $\mathrm{H} \alpha$ flux $F(\mathrm{H} \alpha)$ as $2.2 \times 10^{-13} \mathrm{ergs} \mathrm{cm}^{-2} \mathrm{~s}^{-1}$, not corrected for extinction. They classify the emission spectrum as $\mathrm{H}$ II region type. The uncorrected $\mathrm{H} \alpha$ flux is equivalent to $S_{v}(20)=1.25 \times 10^{12} F(\mathrm{H} \alpha) \quad \mathrm{mJy}=0.27 \mathrm{mJy}$ of optically thin free-free emission if $T_{e}=10^{4} \mathrm{~K}$. We find a $\lambda 20$ $\mathrm{cm}$ peak brightness of $29 \mathrm{mJy}^{\text {beam }}{ }^{-1}$ with $11^{\prime \prime} 4$ resolution.

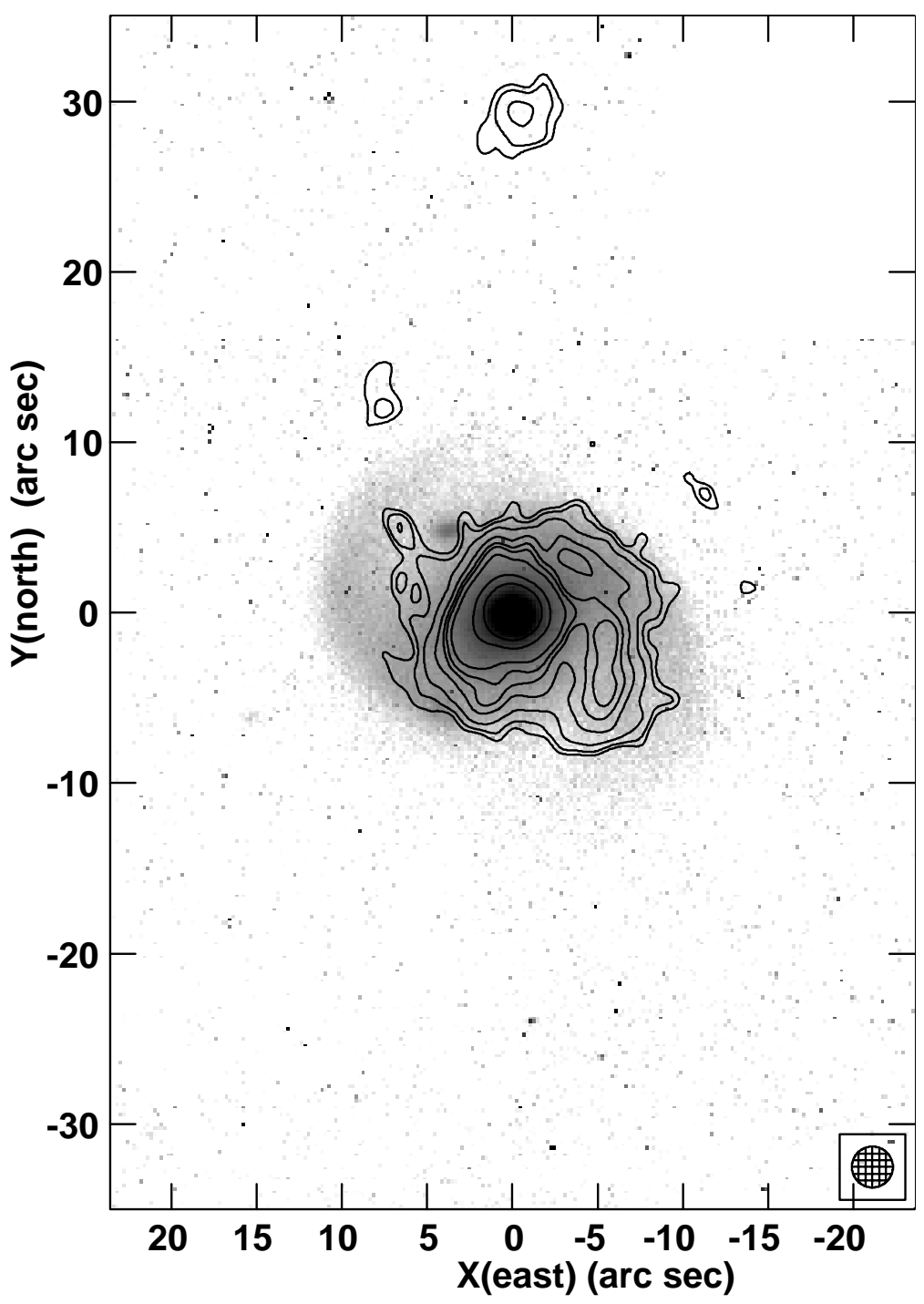

FIG. 4.-Gray-scale display of the $J$-band image of NGC 5394 on a logarithmic intensity scale with contours from the full-resolution $\mathrm{H} \alpha$ image overlaid. The $\mathrm{H} \alpha$ contour levels are at $4,6,10,18,25,30,40,80$, and 120 times the rms noise. Neither the inner spiral arm east of the nucleus nor the westernmost spiral arm is detected in $\mathrm{H} \alpha$. The beam symbol represents the $\mathrm{H} \alpha$ resolution. 


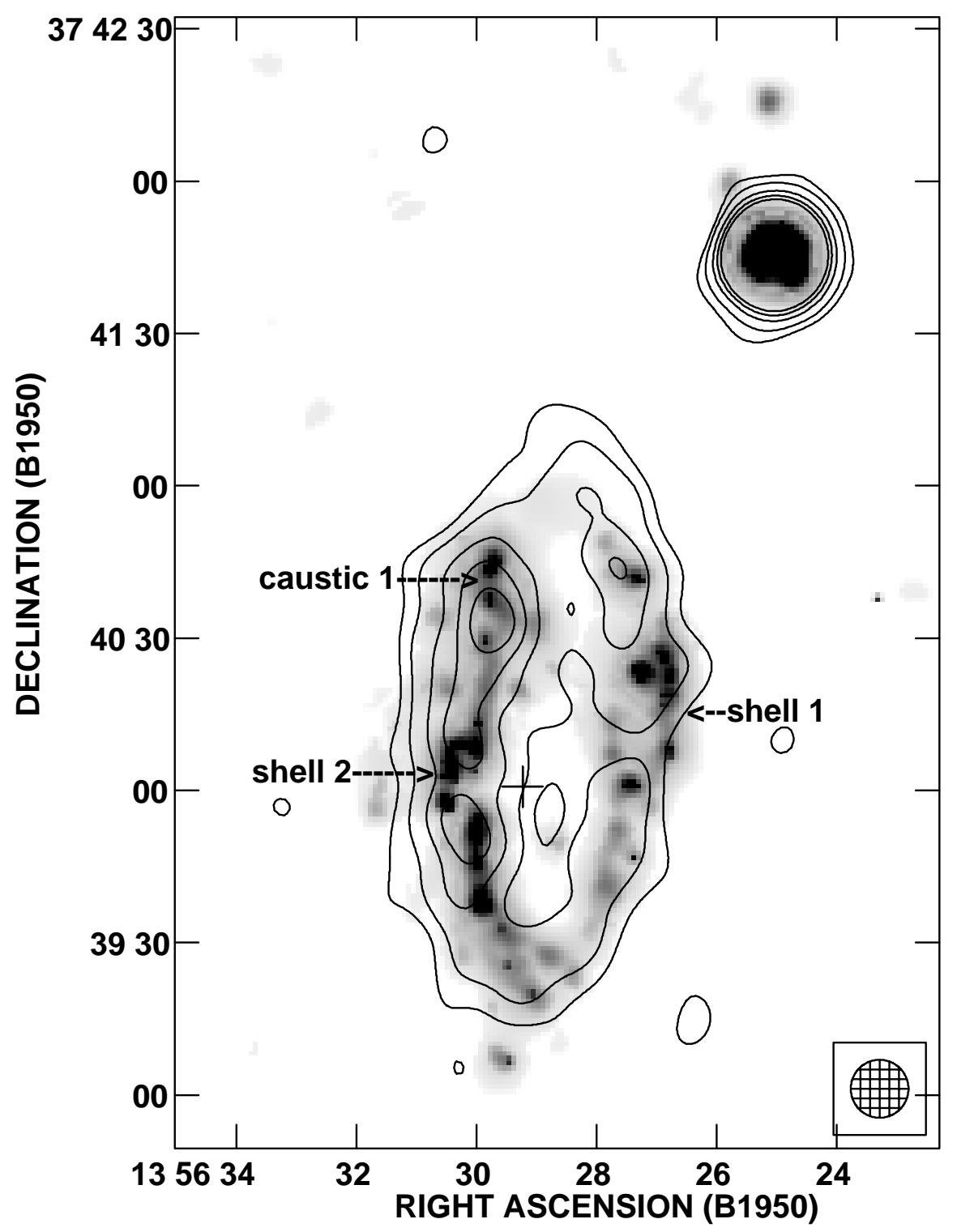

FIG. 5.-Gray-scale display of the composite-resolution $\mathrm{H} \alpha$ image with contours from the high-resolution $\lambda 20 \mathrm{~cm}$ radio continuum image overlaid. The plus sign marks the location of the NGC 5395 nucleus. The beam symbol represents the radio continuum resolution. The rms noise in the radio continuum image is $0.14 \mathrm{mJy}_{\text {beam }}^{-1}$, and the contour levels are at $3,6,12,18$, and 24 times the rms noise.

We get an integrated $S_{v}(20)$ of $33 \mathrm{mJy}$ for NGC 5394 with a deconvolved size of $5^{\prime \prime} \times 4^{\prime \prime}(\mathrm{FWHM})$ so the radio emission is confined to the central part of the galaxy. The FIRST survey (with 4" resolution) lists a peak brightness of 20.5 mJy beam ${ }^{-1}$ and an $S_{v}(20)$ of $29.5 \mathrm{mJy}$ (Becker et al. 1995). The large difference between the measured value of $S_{v}(20)$ and that deduced from the uncorrected $\mathrm{H} \alpha$ flux could result mainly from $\mathrm{H} \alpha$ extinction or mainly from nonthermal radio emission. In $\S 7$, we find a lot of molecular gas in the $33^{\prime \prime}$ aperture centered on the NGC 5394 nucleus, and thus there may be a lot of extinction in the nucleus region. In summary, the $\mathrm{H} \alpha$ emission from NGC 5394 comes from the small, very bright nucleus, from an extended region around the nucleus with a protrusion to the northeast, from the $J$-band spiral arm on the western side that is closer to the nucleus, and from two emission knots on the north tidal arm (see Fig. 4). The Fabry-Perot and the Fick Observatory $\mathrm{H} \alpha$ images detect no emission from the southern tidal arm.

\section{SURFACE PHOTOMETRY OF NGC 5394 IN $J$ AND $R$ BANDS}

The variations of the ellipticity and the position angle of the photometric major axis with radius $r$ (where $r$ is measured in the sky plane) are displayed in Figure 6 for $J$ and $R$ bands. The details in the central few arcseconds are represented better by the $J$-band data than by the $R$-band data because of the superior resolution of the former. NGC 5394 has a small, central, barlike feature with a semimajor axis of $8^{\prime \prime}$. The inner spiral arms end at $r=13^{\prime \prime}$. The major axis position angle varies continuously from about $120^{\circ}$ at small radii where the light is dominated by the bar, to $60^{\circ}$ at the end of the inner spiral arms, to about $0^{\circ}$ in the outer galaxy, where the light is dominated by the tidal arms. In the part of the disk dominated by the inner spiral arms, the ellipticity $\approx 0.35$. If the disk were intrinsically circular in this region, this would imply an inclination $i$ of $20^{\circ}$. From prograde 


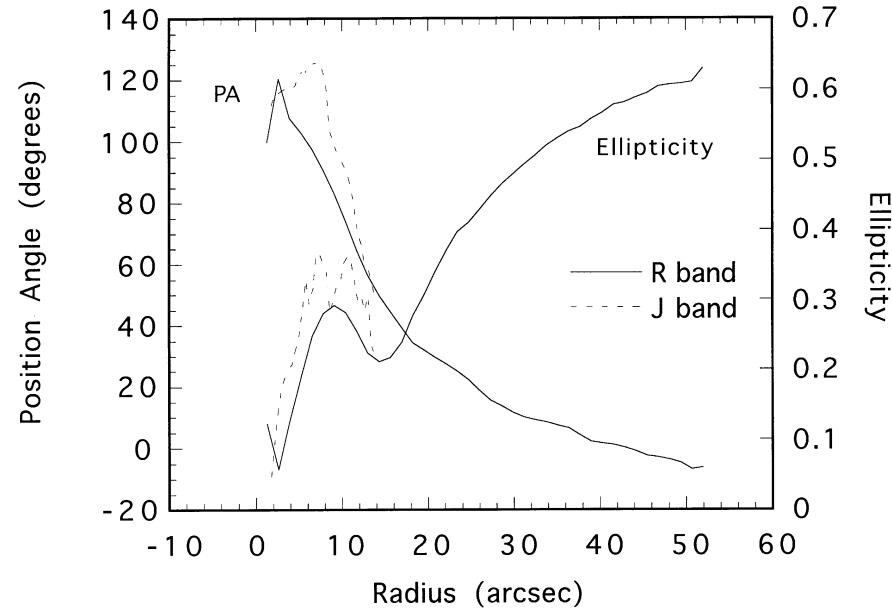

FIG. 6.-Eccentricity and major axis position angle of NGC 5394 as a function of radius $r$ in the plane of the sky.

encounter simulations such as those in Elmegreen et al. (1991) and in $\S 9$ below, it seems unlikely that the central part of the disk is intrinsically circular. This is corroborated by the velocity data presented below, which suggest that the disk is probably being viewed fairly close to face-on. In the region dominated by the tidal arms, the ellipticity increases significantly toward the outer part of the galaxy.

Figure 7 displays the stellar arm-interarm contrast as a function of radius $r$. For the inner spiral arms, the values of the arm/interarm contrast are from the high-resolution $J$-band image. Normal spiral arms have a typical arm contrast of 1-2 mag (Elmegreen \& Elmegreen 1984 for $B$ and $I$ band; Elmegreen et al. 1996 and Regan \& Elmegreen 1997 for $J$ - and $K$-band studies of barred spirals), with armcontrast increasing from the center to a maximum at about $0.5 R_{25}$. For the inner spiral arms of NGC 5394, the observed values of the arm contrast in $J$ band are consistent with normal spiral arms. For the tidal arms, the stellar arm-interarm contrast is best represented by the $R$-band data; the $I$-band image has poorer resolution, and in the $J$-band image, the tidal arms are quite underexposed, leading to an uncertainty in the arm contrast of about 0.5

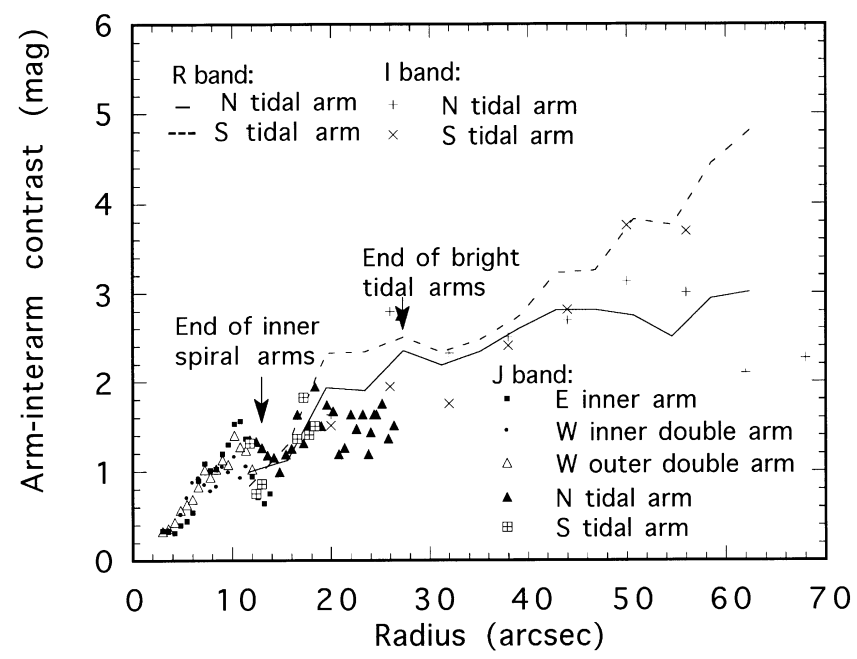

FIG. 7.-Arm-interarm contrast of the inner spiral arms and outer tidal arms of NGC 5394 as a function of radius $r$ in the plane of the sky, as measured on $R$ - and $I$-band optical images and on the near-infrared $J$-band image. mag. In $R$ band, the arm contrast of the tidal arms is $2.5-3$ mag over a large portion of the tidal arms. In this radial range, the interarm surface brightness is clearly above the sky background. The southern arm runs into NGC 5935, so the rise in arm contrast beyond $r=50^{\prime \prime}$ is contaminated. The observed values are larger than for normal spiral arms and are consistent with tidal arms. The values measured in $I$ and $J$ bands match those found in $R$ band within the measurement uncertainties (see Fig. 7). This implies that extinction does not have a significant effect on the arm contrast, for if dust extinction were important, then the observed arm contrast in $J$ band should be greater than in $R$ band. Galaxy encounter simulations, such as those in Elmegreen et al. (1991) and Elmegreen et al. (1995b), predict a large stellar arm-interarm contrast for tidal arms at certain stages of a prograde encounter and thus are consistent with the observed arm contrast of the tidal arms in NGC 5394, IC 2163 (Elmegreen et al. 1995a), and NGC 2535 (Kaufman et al. 1997).

\section{5. $\mathrm{H} \alpha$ VELOCITY FIELD}

\subsection{NGC 5395}

Figure 8 displays $\mathrm{H} \alpha$ isovelocity contours overlaid on the $\mathrm{H} \alpha$ surface-brightness in gray scale; both images were made with composite resolution. The receding side of NGC 5395 is the south side, the near side is the west side, and the rotation is clockwise.

There is an organized pattern of kinks (indicative of streaming motions) in the velocity contours crossing the $\mathrm{H} \alpha$ and radio continuum ring: the velocity kinks are $n$-shaped on the eastern half of the ring, n-shaped on the southwestern quarter of the ring, and u-shaped on the northwestern quarter of the ring. This organized arrangement implies that the velocity kinks result from global mechanisms, not from streaming associated with local star formation. Also, the amplitude of the kinks does not appear to correlate with the $\mathrm{H} \alpha$ surface brightness.

Before considering explanations for the general pattern, we describe the evidence for streaming motions associated with caustic 1 and the two possible shells marked in Fig. 5. In Figure 8, there is an n-shaped kink in the $3320 \mathrm{~km} \mathrm{~s}^{-1}$ velocity contour crossing the eastern side of caustic 1 . This is consistent with expansion in the plane of the disk with a present expansion speed (uncorrected for inclination effects) of $\approx 15 \mathrm{~km} \mathrm{~s}^{-1}$ (= $\left.15 \mathrm{pc} \mathrm{Myr}^{-1}\right)$, but tangential streaming is not ruled out. Note that the kinematics of a caustic feature should differ from those of a superbubble. Shells 1 and 2 have major axes approximately parallel to the major axis of the galaxy, and shell 1 has the same axis ratio as the disk. They appear from the velocity field to be very large expanding shells. Shell 1 has u-shaped velocity kinks on the western side and $n$-shaped velocity kinks on the southeastern side (as expected for shell expansion in the plane of the disk). The interpretation of shell 1 is complicated by the dust lane along the middle of the western arm; shell 1 could be just two strings of $\mathrm{H}$ II regions not in the same plane. With either interpretation, shell 1 is an interesting structure. Shell 2 also has velocity kinks indicative of shell expansion in the plane of the disk, but this shell is less obvious because its western side is absent. Shells 1 and 2 can be seen only in the ionized gas, not as stellar features, whereas caustic 1 is a stellar feature as well as a feature in the ionized gas. Therefore caustic 1 probably had a different origin than shells 1 


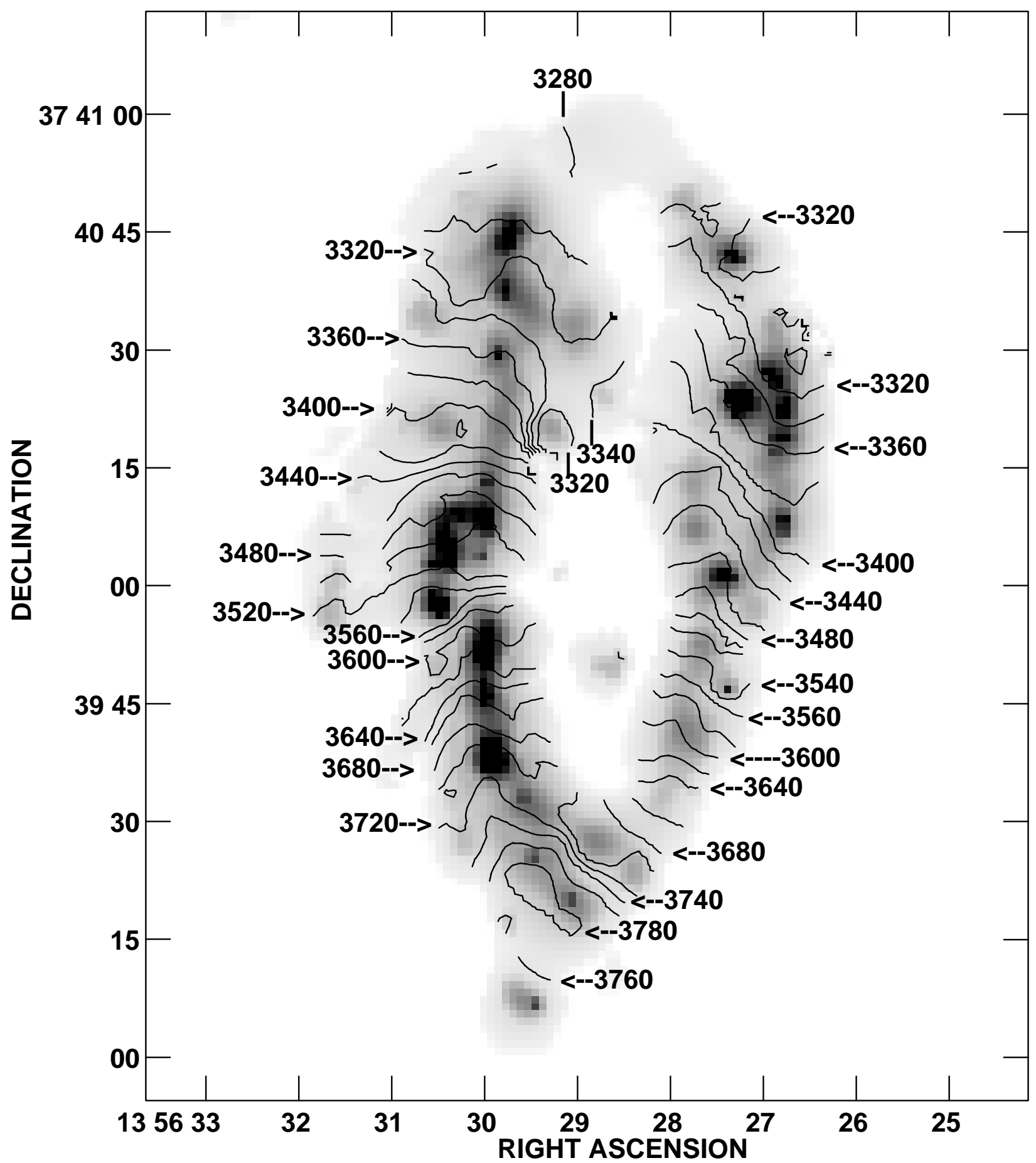

FIG. 8. $-\mathrm{H} \alpha$ isovelocity contours overlaid on the $\mathrm{H} \alpha$ surface brightness image of NGC 5395 in gray scale, both with composite resolution. The contour interval is $20 \mathrm{~km} \mathrm{~s}^{-1}$. Notice the organized pattern of kinks in the velocity contours crossing the $\mathrm{H} \alpha$ and radio continuum ring.

and 2. Shells 1 and 2 may be superbubbles, e.g., the result of tidal debris hitting the disk (Tenorio-Tagle 1981).

The overall kinematics of the $\mathrm{H} \alpha$ and radio continuum ring may be a mixture of ring expansion and the tangential streaming motions from a spiral density wave, but this is all complicated by caustic 1 and the expanding shells. Radial expansion of the ring should produce velocity kinks that are $\mathrm{n}$-shaped on the eastern half of NGC 5395 and u-shaped on the western half. This fits the observed orientations of the velocity kinks in the northeast, southeast, and northwest quadrants, but not in the southwest quadrant, where the kinks south of shell 1 are generally $n$-shaped.

Inside corotation, spiral density wave models (e.g., Visser 1980a, 1980b; Roberts \& Hausman 1984; Sundelius et al. 1987) predict that just after passing the spiral shock front, the gas will move radially inward (toward the galaxy center) and the tangential component of its velocity $v_{t}$ will increase. We assume that the spiral density-wave streaming in NGC 5395 is dominated by the tangential component since radially inward streaming would disagree with the observed 
orientation of the velocity kinks in three of the four quadrants. Velocity kinks produced by an increase in $v_{t}$ should be $n$-shaped in the southeast and southwest quadrants and $\mathrm{u}$-shaped in the northeast and northwest quadrants. Aside from regions affected by local shell or caustic kinematics, this fits the observed orientation of the velocity kinks except in the northeast quadrant, where the kinks between caustic 1 and shell 2 are $n$-shaped.

Perhaps the best explanation for the general pattern of peculiar velocities is a combination of radial expansion of the whole $\mathrm{H} \alpha$ and radio continuum ring and the tangential streaming from a spiral density wave, but this seems to require nonuniform expansion so that radial expansion dominates in the northeast quadrant and spiral densitywave streaming dominates in the southwest quadrant. We shall see in $\S 6$ below that the $\mathrm{H}$ I velocity field in the northeast quadrant is strange.

\subsection{NGC 5394}

Figure 9 displays the isovelocity contours from the composite-resolution $\mathrm{H} \alpha$ velocity field image overlaid on the $J$-band image of NGC 5394 in gray scale. Although the absolute velocity calibration of these data is uncertain by as much as $50 \mathrm{~km} \mathrm{~s}^{-1}$, the value of $3458 \mathrm{~km} \mathrm{~s}^{-1}$ for the FabryPerot $\mathrm{H} \alpha$ velocity at the very bright, sharp nucleus of NGC 5394 does not differ much from Karachentsev's (1980) longslit value of $3451 \pm 12 \mathrm{~km} \mathrm{~s}^{-1}$ (see Table 1). Since the receding side is the north side and the rotation is counterclockwise (for trailing arms), the near side of NGC 5394 is the west side. Note that NGC 5394 and NGC 5395 are rotating in opposite senses; in both galaxies the west side is the near side. The $\mathrm{H} \alpha$ velocity difference across NGC 5394 is small; the velocities go from $3417 \mathrm{~km} \mathrm{~s}^{-1}$ on the western $J$-band spiral arm to $3477 \mathrm{~km} \mathrm{~s}^{-1}$ in the region northeast of the nucleus. The $\mathrm{H} \alpha$ knot $30^{\prime \prime}$ north of the

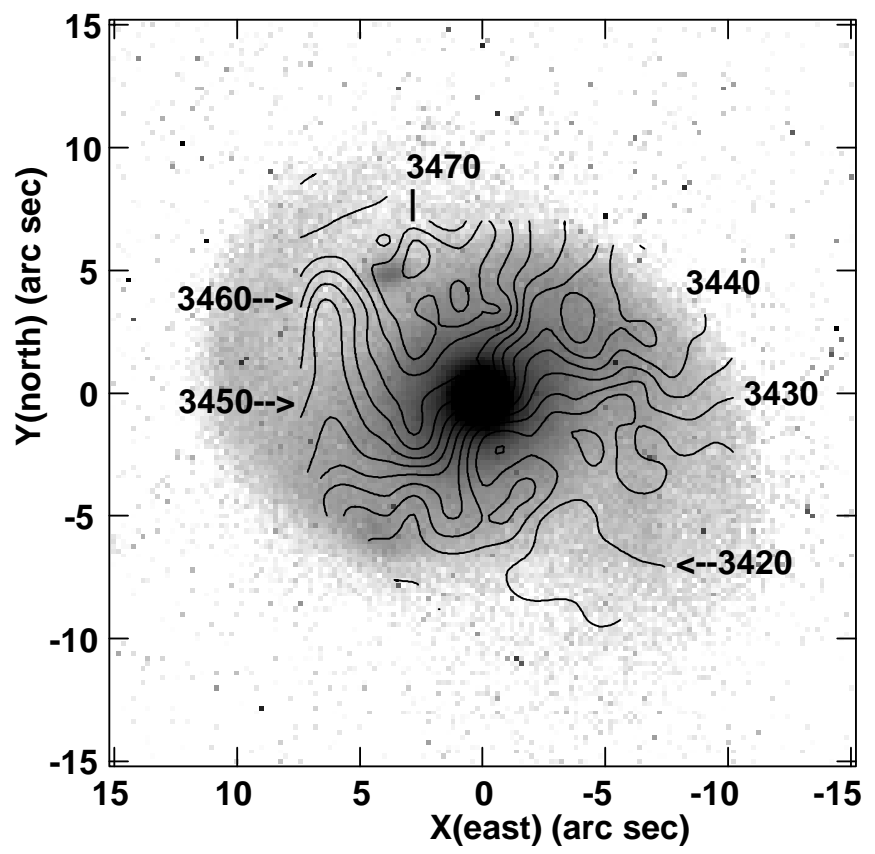

FIG. 9.- Isovelocity contours from the composite-resolution $\mathrm{H} \alpha$ velocity image overlaid on the $J$-band image of NGC 5394 in gray scale. The contour interval is $5 \mathrm{~km} \mathrm{~s}^{-1}$. The large n-shaped velocity kinks $8^{\prime \prime}$ northeast of the nucleus occur in an interarm $\mathrm{H} \alpha$ feature. nucleus is not shown in this figure; it has a velocity of 3460 $\mathrm{km} \mathrm{s}^{-1}$. The kinematic minor axis is hard to identify in this image because of noncircular motions. There are u-shaped kinks in the velocity contours $3^{\prime \prime}$ southeast of the nucleus and n-shaped velocity kinks $3^{\prime \prime}$ northwest of the nucleus. It is not clear what causes these; they may be an indication of a circumnuclear ring or a bar within a bar. The $J$-band image has no obvious feature at $3^{\prime \prime}$ from the nucleus, but the $R$-band ellipticity plot in Figure 6 shows a change in ellipticity and position angle here.

The following features suggest that there may be a (somewhat) collimated outflow of ionized gas from the starburst in the nucleus. It appears as an elongated $(6 \times \mathrm{rms}$ noise) $H \alpha$ feature northeast of the nucleus (see Fig. 4) in the region between the nucleus and the inner $J$-band spiral arm. There are large $\mathrm{n}$-shaped velocity kinks at the location of this interarm $\mathrm{H} \alpha$ protrusion $\left(8^{\prime \prime}=1.8 \mathrm{kpc}\right.$ northeast of the nucleus), with no corresponding velocity kinks visible on the opposite side of the nucleus (see Fig. 9). If the northeast velocity kinks represent outflow of ionized gas from the nucleus, then the motion is out of the disk and toward us at $\sim 10 \mathrm{~km} \mathrm{~s}^{-1}$ in projection. The outflow may be one-sided, or dust extinction may be occulting the $\mathrm{H} \alpha$ emission from a counterflow on the far side. Figure 10 shows that the $\mathrm{H} \alpha$ line profile at the nucleus has a blue wing reaching to 150 $\mathrm{km} \mathrm{s}^{-1}$ from the central velocity. This may be part of the same wind/outflow phenomenon. In the Fabry-Perot data, $[\mathrm{N} \mathrm{II}]$ gets aliased $50 \mathrm{~km} \mathrm{~s}^{-1}$ to the blue of $\mathrm{H} \alpha$. Based on the attenuation by the blocking filter at the velocity of the nucleus and the measured $[\mathrm{N} \mathrm{III} / \mathrm{H} \alpha$ ratio of 0.53 (Keel et al. $1985)$ and assuming that the $[\mathrm{N} \mathrm{II}] / \mathrm{H} \alpha$ ratio remains constant at each velocity in the line, the $[\mathrm{N}$ II $]$ alias should be at least 10 times weaker than $\mathrm{H} \alpha$ here, so aliased [N II] is not the main cause of the extended blue wing. Arp (1969) estimated the age of the starburst as $\approx 10^{7}$ years. The starburst and outflow in NGC 5394 may be similar to that in NGC 2782 (Jogee, Kenney, \& Smith 1998). In both cases, the age of the starburst is younger than the dynamical timescale of

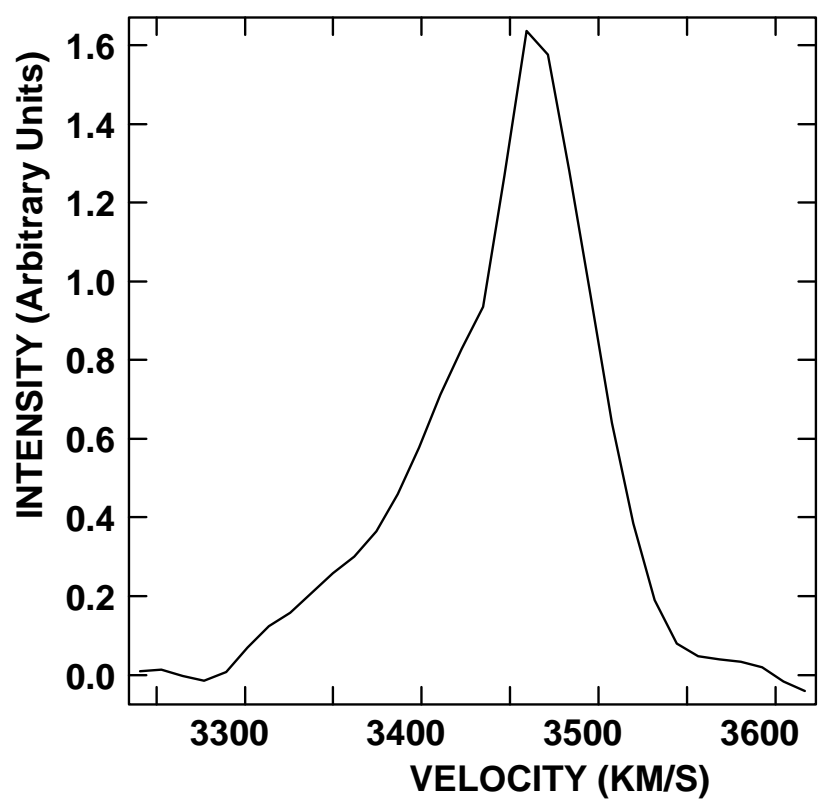

FIG. 10.- $\mathrm{H} \alpha$ line profile at the NGC 5394 nucleus. The profile has an extended blue wing that may represent outflow. 
the collision, and the kiloparsec length scales and $10 \mathrm{Myr}$ timescales of the outflow imply time-averaged outflow velocities of $\sim 100 \mathrm{~km} \mathrm{~s}^{-1}$. In NGC 5394, this is consistent with the present $100 \mathrm{~km} \mathrm{~s}^{-1}$ velocity asymmetry of the nuclear line profile.

\section{H I PROPERTIES}

\subsection{Combined H I Emission of the System}

Figure 11 displays channel maps (every other channel) made from the $\mathrm{H}$ I cube with low resolution $\left(18^{\prime \prime} 4 \times 17^{\prime \prime} .7\right)$ before we attempted to separate the contributions of the two galaxies. These maps are overlaid on the Digitized Sky Survey image ${ }^{13}$ in gray scale. $\mathrm{H}$ I emission from the larger galaxy NGC 5395 is present over the velocity range 3125 $3810 \mathrm{~km} \mathrm{~s}^{-1}$. One can see from these channel maps that strong H I emission from NGC 5395 extends for a considerable distance north and northeast of the optical galaxy. There is a fainter, diffuse $\mathrm{H}$ I extension $3^{\prime}$ south-southwest of NGC 5395. The south-southwest extension was detected in the VLA D configuration $\mathrm{H}$ I observations by Nordgren et al. (1997) with 54" resolution, but their data were plagued by solar interference problems. The channel maps show H I emission from the direction of NGC 5394.

For the two galaxies combined, Figure 12 is a gray-scale display of the $\mathrm{H}$ I column density $N(\mathrm{H}$ I) made from the low-resolution cube $\left(18^{\prime \prime} 4 \times 17^{\prime \prime} 7\right)$. Throughout this paper, $N(\mathrm{H}$ I) denotes the line-of-sight column density, and we assume the $\mathrm{H} \mathrm{I}$ emission is optically thin. The brightest $\mathrm{H} \mathrm{I}$ emission from NGC 5395 forms a large ring that coincides with the $\mathrm{H} \alpha$ and radio continuum ring found in $\S 3.2$ above (see Fig. 13, where H I contours are overlaid on the lowresolution $\lambda 20 \mathrm{~cm}$ continuum image in gray scale). The western side of the ring is brighter in $\mathrm{H} \mathrm{I}$ emission, whereas the eastern side of the ring is brighter in the radio continuum and has a greater number of highly luminous $\mathrm{H}$ II regions. We suspect from the anticorrelation that there is an important molecular component on the eastern side of the ring, but need sensitive, high-resolution ${ }^{12} \mathrm{CO}$ observations to confirm this. There is $\lambda 20 \mathrm{~cm}$ continuum emission from the region between the two galaxies at the $8 \times$ rms noise level (see Fig. 13).

\subsection{Separation of the $\mathrm{H}$ I Contributions of the Two Galaxies}

To separate the $\mathrm{H}$ I contributions of the two galaxies, we inspected sets of position-velocity maps choosing the abscissa first along the declination axis and then along the right ascension axis. These orientations were chosen because the photometric major axis of NGC 5395 deviates by only a few degrees from the declination axis. Figure 14 displays four declination-velocity diagrams spaced at $15^{\prime \prime}$ intervals in right ascension, starting at the center of the NGC 5395 ring and ending at the center of NGC 5394. The set of declination-velocity diagrams makes clear that, based on the kinematics, one can discern three components to the $\mathrm{H}$ I emission: the disk of NGC 5394, the main disk of NGC 5395 , and a long, narrow H I tidal arm of NGC 5395 distinct in velocity from its main disk. We shall call the tidal arm with the peculiar velocity the northern $\mathrm{H}$ I tidal arm of NGC 5395. At the same projected location, this tidal arm

\footnotetext{
${ }^{13}$ The POSS Digitized Sky Survey was produced at the Space Telescope Science Institute under grant NAG W-2166.
}

has a greater line-of-sight velocity than the main disk of NGC 5395 and deviates by as much as $75-100 \mathrm{~km} \mathrm{~s}^{-1}$ from it. This velocity difference at the same projected location implies that the northern $\mathrm{H}$ I tidal arm is not in the same plane as gas in the disk; otherwise it would collide with the disk gas and produce a strong shock. From the $\mathrm{H}$ I cube of the combined emission, we extracted, separately, the northern $\mathrm{H}$ I tidal arm of NGC 5395 and the $\mathrm{H}$ I emission of NGC 5394. This resulted in three $\mathrm{H}$ I cubes and associated sets of moment maps: (1) a cube for NGC 5394, (2) a cube for the northern $\mathrm{H}$ I tidal arm of NGC 5395, and (3) a cube for NGC 5395 after removal of the northern $\mathrm{H}_{\mathrm{I}}$ tidal arm with the peculiar velocity. This last cube contains any residual features left after removing the emission associated with NGC 5394 and the northern $\mathrm{H}$ I tidal arm of NGC 5395. In extracting the northern H I tidal arm of NGC 5395 from the cube of the combined emission, we could not follow the southern end of this arm beyond where it overlaps the main disk in velocity.

At the location of NGC 5394, there is some $\mathrm{H}$ I gas at a velocity of $3700 \mathrm{~km} \mathrm{~s}^{-1}$ (see the channel maps in Fig. 11), about $200 \mathrm{~km} \mathrm{~s}^{-1}$ higher in line-of-sight velocity than gas that clearly belongs to NGC 5394 with nothing connecting it in velocity to the NGC 5394 gas. We omitted this highvelocity cloud from the H I cube for NGC 5394 alone. No $\mathrm{H} \alpha$ emission is associated with the high-velocity cloud. This cloud could be tidal debris from NGC 5395 falling onto NGC 5394 or material stripped out of NGC 5394 by the interaction. The $\mathrm{H}$ I mass of this cloud $\left(6 \times 10^{7} M_{\odot}\right)$ is comparable to the $\mathrm{H}$ I masses of massive $\mathrm{H}$ I clouds found in arm I on the western side of NGC 5395 and in other interacting systems (see discussion in $\S 6.3 .1$ below). The galaxy encounter simulation in Elmegreen, Kaufman, \& Thomasson (1993) suggests that clouds of this mass may escape the parent galaxy to form a future dwarf galaxy. If a highvelocity cloud of this mass were to hit the disk, it might produce a giant shell (Tenorio-Tagle 1981), such as shells 1 and 2 in NGC 5395. In the direction of NGC 5394, the channel maps contain a few weak features at other velocities (e.g., $3200 \mathrm{~km} \mathrm{~s}^{-1}$ ) that may be other high-velocity clouds, but these are considerably weaker than the $3700 \mathrm{~km} \mathrm{~s}^{-1}$ emission.

Figures 15, 16, and 17, display the low-resolution, total column density maps of each component, separately, as contours overlaid on an optical image in gray scale.

The declination-velocity diagrams of the eastern side of NGC 5395 (see Figure 18) reveal three distinct velocity features in the northern part of this galaxy: the northern $\mathrm{H} \mathrm{I}$ tidal arm of NGC 5395, a feature labelled the "NNE" (north-northeast) extension in Figure 15, and the main disk. Thus the northern $\mathrm{H}$ I tidal arm is not the only nonplanar structure north-northeast of the $\mathrm{H} \alpha$ and radio continuum ring. Faint optical emission is seen from part of the northnortheast extension (compare Figs. 15 and 17). No distinct anomaly in the P-V diagram at the location of caustic 1 is visible (see bottom panel of Fig. 18, which includes the eastern ridge of caustic 1); caustic 1 is unresolved at the spatial resolution of the $\mathrm{H}$ i data.

For comparison with the model in $\S 9$, Figure 19 presents a declination-velocity diagram in which the $\mathrm{H}$ I emission from the system is summed over all the values of RA displayed in Figure 13. This shows that the diffuse $\mathrm{H}$ I emission south-southwest of NGC 5395 connects in velocity to the southern part of the main disk of NGC 5395. We shall refer 


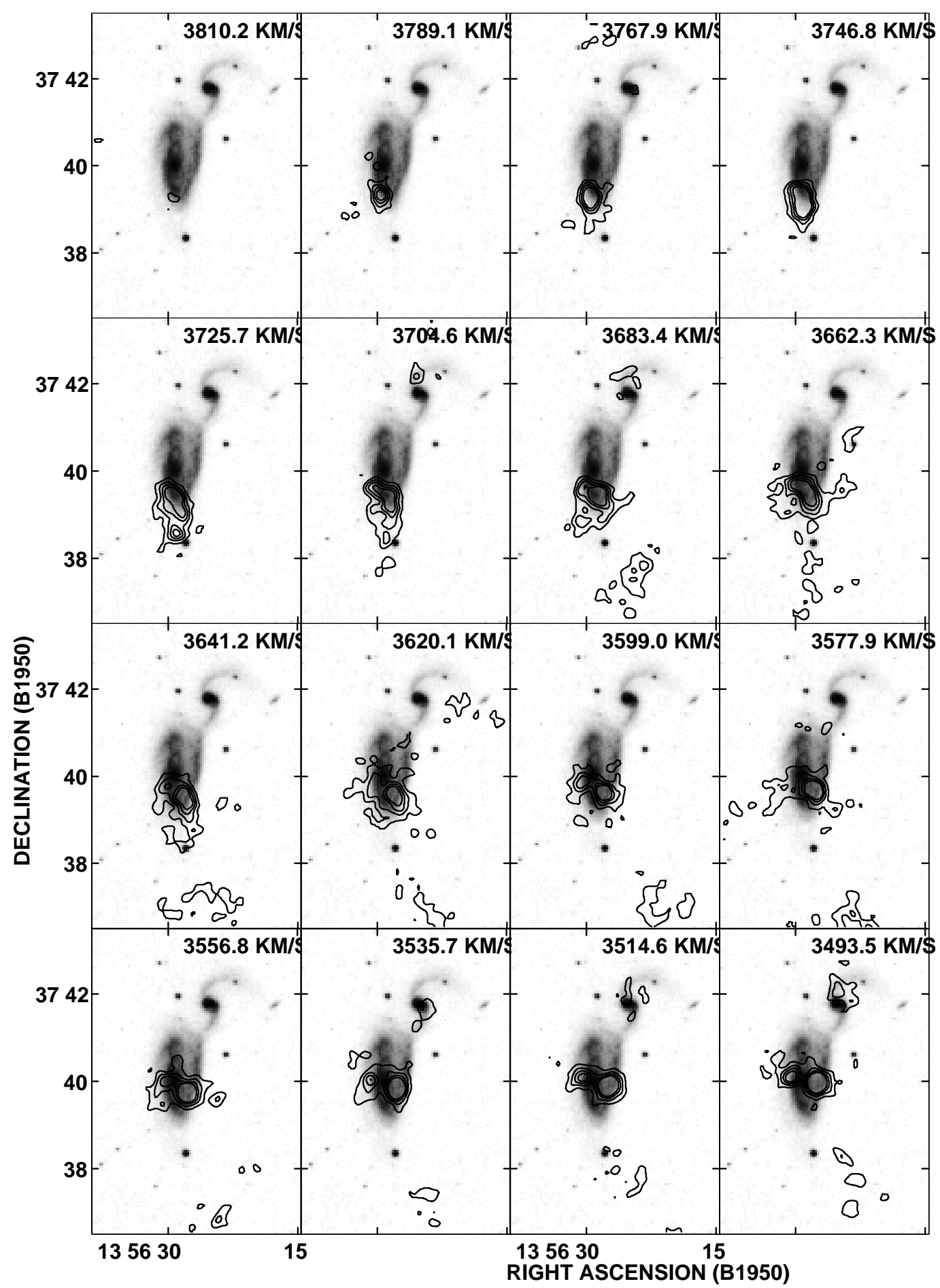

FIG. $11 a$

Fig. 11.- Set of channel maps (every other channel) from the low-resolution $\left(18^{\prime \prime} .4 \times 17^{\prime \prime} .7\right) \mathrm{H}$ I cube overlaid on a gray-scale display of the POSS Digitized Sky Survey image. The rms noise in the channel maps is $0.46 \mathrm{mJy}_{\text {beam }}^{-1}$ and the contours levels are at 2, 4, 6, and 8 times the rms noise.

to this feature as the broad, diffuse, southern tidal arm of NGC 5395. Its characteristics differ from those of the northern H I tidal arm of NGC 5395. As Figure 16 shows, the latter is a long, narrow arm which has high surface brightness where it appears to join the disk in projection. The southern tidal arm, on the other hand is a broad, diffuse feature with only weak emission where it connects in position to the disk (see Fig. 13). Table 4 lists the H I masses (excluding helium) of the various components of this system. Although there is some uncertainty as to where to place the dividing line between the ring in NGC 5395 and the $\mathrm{H}$ I emission north and east of the ring, it appears that the H I mass of the north-northeast extension of NGC 5395 is roughly equal to that of the northern $\mathrm{H}$ i tidal arm of NGC 5395 and also roughly equal to that of the broad, diffuse, southern tidal arm of NGC 5395. In NGC 5395, the 


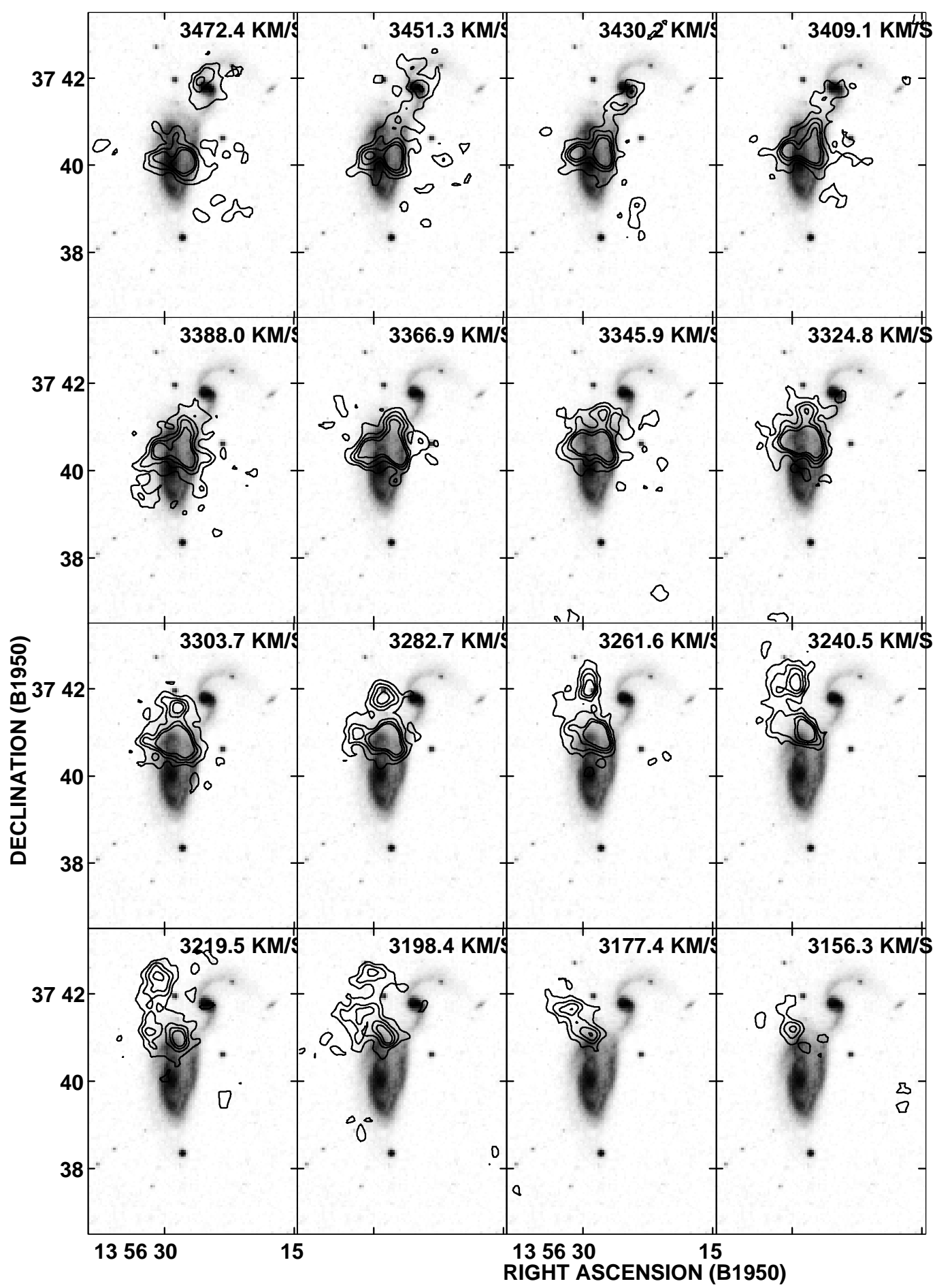

FIG. $11 b$

northern $\mathrm{H}$ I tidal arm, the broad, diffuse, southern tidal arm, and the north-northeast extension together comprise about $30 \%$ of the total $\mathrm{H}$ I mass of the galaxy.

\subsection{The Disk of NGC 5395 After Removal of the Northern H I Tidal Arm \\ 6.3.1. Morphology and Massive Clouds}

The $\mathrm{H} \mathrm{I}$ ring is the dominant feature of the $\mathrm{H}$ I disk in NGC 5395. In Figure 15, we have marked a small H I structure that corresponds to the northern end of the Arp blue arm. It is unlikely to be contamination by NGC 5394 as the two galaxies should differ in velocity here by about $100 \mathrm{~km} \mathrm{~s}^{-1}$, but it is possibly the result of some residual contamination by the northern $\mathrm{H}$ I tidal arm.

Figure 20 overlays the high-resolution (11".4 FWHM) H I column density image as contours on the compositeresolution $\mathrm{H} \alpha$ image in gray scale. Five $\mathrm{H}$ I cloud complexes, each with $\mathrm{H}$ I mass in excess of $10^{8} \mathrm{M}_{\odot}$, are labelled in 


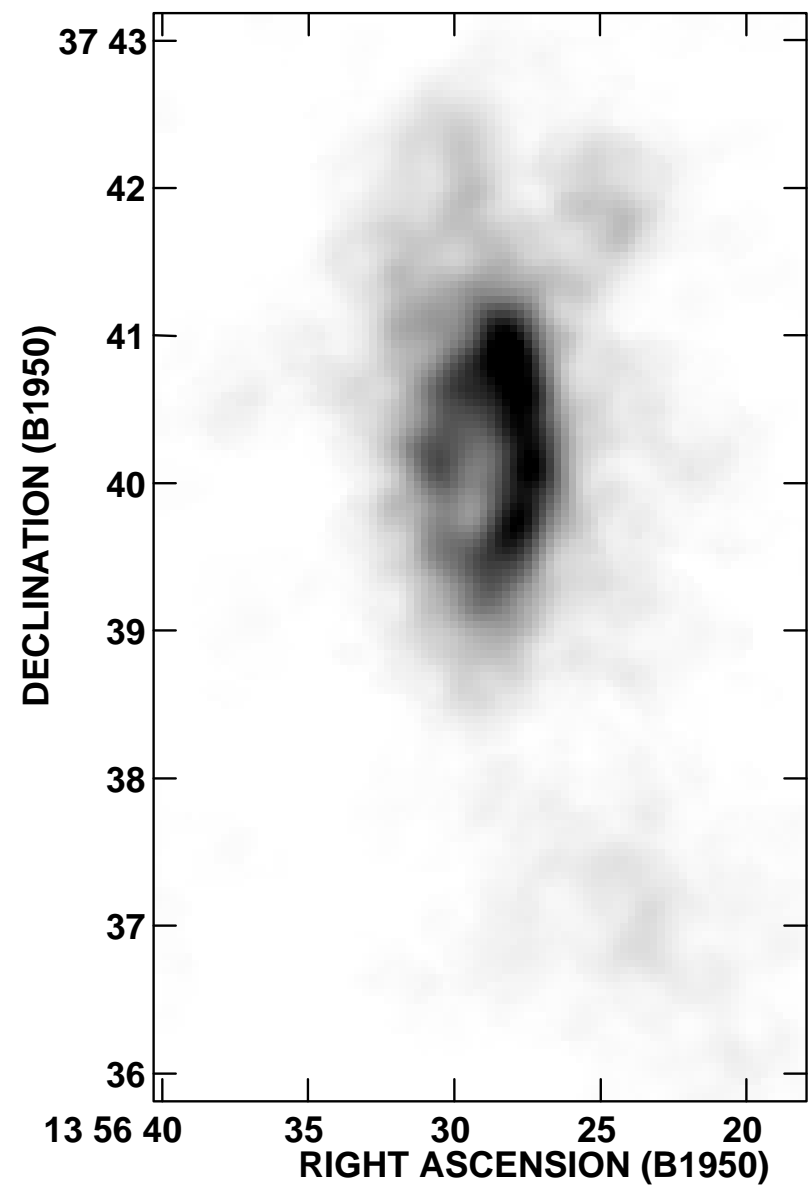

FIG. 12. - Gray-scale display of the H I column density of the two galaxies combined, made from the low-resolution $\left(18^{\prime \prime} 4 \times 17^{\prime \prime} .7\right)$ data.

this figure. Table 5 lists the following properties of these $\mathrm{H} \mathrm{I}$ clouds: the position, the average $\mathrm{H}$ I column density $N(\mathrm{H} \mathrm{I})$, the total $\mathrm{H}$ I mass $M(\mathrm{H} \mathrm{I})$ of the cloud (excluding helium), and the cloud semimajor axis $a$ (computed as described in Kaufman et al. 1997). Since $N(\mathrm{H}$ I $)$ is the column density along line of sight, the average face-on surface density $\mu(\mathrm{H} \quad \mathrm{I})=N(\mathrm{H} \quad \mathrm{I}) \cos (i)=13-14 \times 10^{20}$ atoms $\mathrm{cm}^{-2}$ in these clouds. Most of these clouds do not coincide with the most luminous $\mathrm{H}$ iI regions in the galaxy. They have $\mathrm{H}$ I masses comparable to the massive $\mathrm{H}$ I clouds in the interacting galaxy pairs IC 2163/NGC 2207 (Elmegreen et al. 1995a), NGC 2535/36 (Kaufman et al. 1997), NGC 5774/75 (Irwin 1994), in the five merger pairs studied by Hibbard
TABLE 4

HI MASSES OF VARIOUS COMPONENTS

\begin{tabular}{|c|c|}
\hline Component & $\begin{array}{c}M\left(\mathrm{H} \mathrm{I}_{\mathrm{I}}\right) \\
\left(M_{\odot}\right)\end{array}$ \\
\hline NGC 5394 & $7.3 \times 10^{8}$ \\
\hline High-velocity cloud near NGC 5394 & $6 \times 10^{7}$ \\
\hline NGC 5395 ring plus interior to ring.. & $1.0 \times 10^{10}$ \\
\hline Northern H I tidal arm of NGC $5395 \ldots$ & $1.3 \times 10^{9}$ \\
\hline NNE extension of NGC $5395 \ldots \ldots \ldots \ldots$ & $1.5 \times 10^{9}$ \\
\hline Broad, diffuse, southern tidal arm of NGC $5395 \ldots \ldots$ & $1.2 \times 10^{9}$ \\
\hline
\end{tabular}

(1995), and in NGC 2782, an isolated galaxy whose peculiar structure appears to result from a prior collision or merger (Smith 1994).

\subsubsection{H I Velocity Field}

In Figure 21, the $\mathrm{H}$ I low-resolution isovelocity contours are overlaid on the Digitized Sky Survey POSS image in gray scale. The velocity field does not possess large-scale reflection symmetry about the kinematic minor axis. There is also no clear $S$-type distortion of the velocity field and thus no evidence of a strong static warp. However, there are probably $z$-motions in the northern part of the disk since the declination-velocity diagrams in $\S 6.2$ show indications of nonplanar structures north of the ring. The velocity field in the northeast quadrant of the galaxy is strange, as the velocity contours do not have the upward curvature expected for rotation. Some speculations about this will be presented below. Although part of this quadrant is perturbed by caustic 1 (see the $\mathrm{H} \alpha$ velocity field in Fig. 8), the strange $\mathrm{H}$ I velocity field in this quadrant is not just the result of unresolved velocity kinks at the caustic or the ring (note that the $\mathrm{H}$ I velocity field in the northeast quadrant extends $20^{\prime \prime}-30^{\prime \prime}$ east of the ring).

The velocity field of the rest of the galaxy appears more normal. The $\mathrm{H}$ I velocity at the nucleus is $3496 \pm 17 \mathrm{~km} \mathrm{~s}^{-1}$ (where we assign a rather large uncertainty to the velocity because of the steep velocity gradient and the uncertainty in the position of the nucleus). This is consistent with the optical value of $3493 \pm 10 \mathrm{~km} \mathrm{~s}^{-1}$ measured by Karachentsev (1980). The $\mathrm{H}$ I isovelocity contour through the nucleus is curved. If this is the kinematic minor axis, then there are strong noncircular motions throughout the galaxy. Instead, we adopt as the $\mathrm{H}$ I kinematic minor axis the straight-line velocity contour with $v=3450 \pm 10 \mathrm{~km}$ $\mathrm{s}^{-1}$. It has a position angle of $83^{\circ}$ (consistent with the position angle of the photometric minor axis) and is located

TABLE 5

Massive H i Clouds IN NGC 5395

\begin{tabular}{|c|c|c|c|c|c|}
\hline Cloud $^{\mathrm{a}}$ & $\begin{array}{c}\alpha \\
(1950.0)\end{array}$ & $\begin{array}{c}\delta \\
(1950.0)\end{array}$ & $\begin{array}{c}N(\mathrm{H} \mathrm{I}) \\
\left(10^{20} \text { atoms cm } \mathrm{cm}^{-2}\right)\end{array}$ & $\begin{array}{c}M(\mathrm{H} \mathrm{I}) \\
\left(10^{8} M_{\odot}\right)\end{array}$ & $\begin{array}{c}a \\
(\mathrm{kpc})\end{array}$ \\
\hline H i $\mathrm{C} 1 \ldots .$. & 135628.2 & 374054 & 32 & 5.7 & 3.6 \\
\hline $\mathrm{H}_{\text {I }} \mathrm{C} 2 \ldots .$. & 135627.7 & 374036 & 33 & 3.8 & 2.6 \\
\hline H i C3...... & 135628.0 & 374021 & 31 & 3.3 & 2.4 \\
\hline H i C4...... & 135627.2 & 374006 & 30 & 4.1 & 3.0 \\
\hline H I C $5 \ldots . .$. & 135627.7 & 373948 & 32 & 4.3 & 2.9 \\
\hline
\end{tabular}

NoTE.-Units of right ascension are hours, minutes, and seconds, and units of declination are degrees, arcminutes, and arcseconds.

a In Fig. 20, the labels are abbreviated to "C1," etc. 


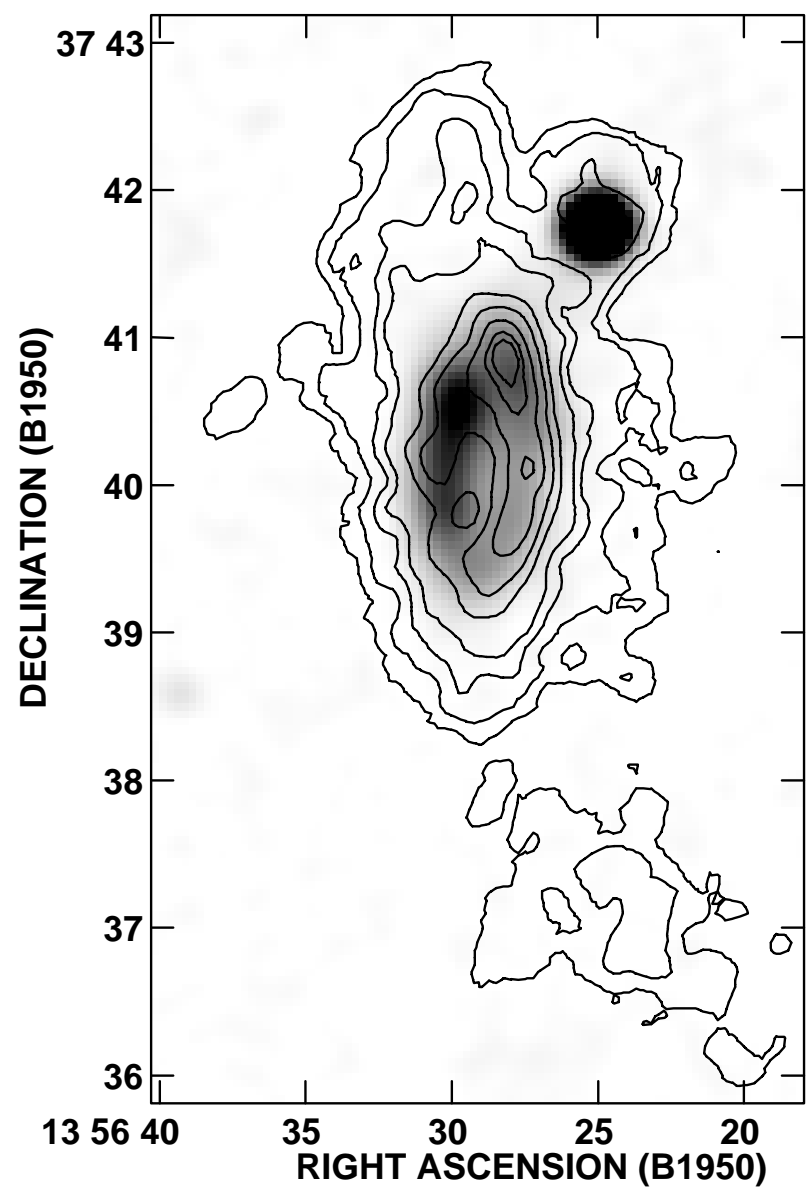

FIG. 13.-Gray-scale display of the $\lambda 20 \mathrm{~cm}$ radio continuum emission overlaid with $N(\mathrm{H}$ I $)$ contours, both from the low-resolution data. The line-of-sight column density contour levels are at 1.7, 3.4, 6.8, 10, 19, 26, 34, $41,44 \times 10^{20}$ atoms $\mathrm{cm}^{-2}$.

$10^{\prime \prime} \pm 2 "$ north of the nucleus. This implies that the dynamical center has moved from the optical nucleus to the center of the $\mathrm{H} \alpha$ and radio continuum ring as a result of the interaction, and $v_{\text {sys }}=3450 \pm 10 \mathrm{~km} \mathrm{~s}^{-1}$. The kinematic major axis on the receding (southern) side has a position angle of $170^{\circ}$ relative to the center of the ring (or $174^{\circ}$ relative to the nucleus). The orientations of the kinematic major and minor axes are consistent with the position angle of the photometric major axis; we adopt $173^{\circ}$ as the position angle of the line of nodes of the projection. Since the galaxy appears somewhat lopsided relative to the optical nucleus in the broadband optical images, it is plausible that the mass distribution is not exactly centered at the nucleus.

The declination-velocity diagram in the upper left panel in Figure 14 goes through the ring center and deviates only $7^{\circ}$ from the major axis of NGC 5395. It indicates two important properties of the velocities in the disk: (1) the rotation curve is still rising at the ring, and (2) there is a north-south asymmetry in velocities relative to $v_{\text {sys. }}$. The north-south asymmetry along the major axis is also seen in the $\mathrm{H}$ I velocity field image. With distance measured from the center of the ring, a slice (see Fig. 22) along the kinematic major axis of the $\mathrm{H}$ I velocity field gives $\left(v_{\max }\right) \sin \mathrm{i} \equiv$ maximum value of $\left|v_{\text {obs }}-v_{\text {sys }}\right|=285 \pm 10 \mathrm{~km} \mathrm{~s}^{-1}$ on the receding side at $r=54^{\prime \prime}-63^{\prime \prime}$ and $\left(v_{\max }\right) \sin i=206 \pm 10 \mathrm{~km}$ $\mathrm{s}^{-1}$ on the approaching side at $r=62^{\prime \prime}-68^{\prime \prime}$ (i.e., just beyond the ring). The $79 \pm 14 \mathrm{~km} \mathrm{~s}^{-1}$ discrepancy implies that non- circular motions are present on the major axis. Out to $r=40^{\prime \prime}$, the velocity gradient is $5.6 \mathrm{~km} \mathrm{~s}^{-1}$ per arcsec on the receding side but only $3.8 \mathrm{~km} \mathrm{~s}^{-1}$ per arcsec on the approaching side. Sharp \& Keel (1985) obtained a long-slit absorption-line spectrum through the nucleus at P.A. $=0^{\circ}$; if the radial distance scale in their "rotation" curve is shifted north by $10^{\prime \prime}$ so that the distances refer to the ring center instead of the nucleus, then it has the same type of north-south asymmetry in velocities as our $\mathrm{H}$ I data. Their spectrum has steeper velocity gradients in the center of the galaxy than our H I velocity field, because of the superior spatial resolution of the slit spectrum.

The north-south asymmetry in velocities along the major axis leads us to suspect that the gas is travelling in elliptical rather than circular orbits, with higher tangential speeds on the receding side than on the approaching side. Although the encounter may have also elongated the disk along the kinematic major axis, we shall continue to use $i=65^{\circ}$. A rotation curve that rises more steeply on one side of a galaxy than on the other is typical of an $m=1$ perturbation to the potential (Schoenmakers \& Swaters 1999). Another possible explanation for the north-south velocity asymmetry on the major axis of NGC 5395 is $z$-motions away from us on both the approaching side and the receding side.

The $H$ I value of $\left(v_{\max }\right) \sin i=285 \mathrm{~km} \mathrm{~s}^{-1}$ on the receding side is high compared to that expected for pure circular motion in a normal galaxy of the same luminosity. The $B_{T}^{0}$ magnitude of NGC 5395 is 12.01 , equivalent to $M_{B}=$ -21.35 and $L_{B}=5.1 \times 10^{10} \quad L_{B} \odot$ if $H_{0}=75 \mathrm{~km} \mathrm{~s}^{-1}$ $\mathrm{Mpc}^{-1}$. From a comparison of rotation curves and luminosities of undisturbed spiral galaxies, Persic \& Salucci (1991) find $v_{\max }=200\left(L_{\boldsymbol{B}} / L_{B}^{*}\right)^{1 / 4} \mathrm{~km} \mathrm{~s}^{-1}$, where (after adjustment to $\left.H_{0}=75 \mathrm{~km} \mathrm{~s}^{-1} \mathrm{Mpc}^{-1}\right) L_{B}^{*}=2.7 \times 10^{10} L_{B \odot}$. The scatter in the Persic-Salucci relation is smaller than in the Tully-Fisher relation and leads to an uncertainty of $\pm 14 \%$ in the predicted value of $v_{\max }$. For an undisturbed spiral with the same luminosity as NGC 5395, the Persic-Salucci relation predicts $v_{\max }=235 \pm 32 \mathrm{~km} \mathrm{~s}^{-1}$. So for $i=65^{\circ}$, one expects $\left(v_{\max }\right) \sin i=213 \pm 30 \mathrm{~km} \mathrm{~s}^{-1}$.

The Arp blue arm does not appear to have an unusual velocity in the $\mathrm{H}$ I velocity-field image. The brightest part of the broad, diffuse, southern tidal arm of NGC 5395 (see Fig. 13) is $3^{\prime}$ south-southwest of the center of the ring and is not included in Figure 21; it has mean velocities of 3560-3600 $\mathrm{km} \mathrm{s}^{-1}$.

In general (e.g., Mihalas \& Binney 1981),

$$
\begin{aligned}
v_{\mathrm{obs}}-v_{\mathrm{sys}}= & v_{\mathrm{c}} \cos \theta \sin i+\Delta v_{\mathrm{t}} \cos \theta \sin i \\
& -v_{\mathrm{r}} \sin \theta \sin i+v_{\mathrm{z}} \cos i,
\end{aligned}
$$

where $v_{c}$ is the circular velocity, $\Delta v_{t}$ is the excess tangential velocity, $v_{r}$ is the expansion velocity, $v_{z}$ is the velocity component perpendicular to the disk, and $\theta$ is the azimuthal angle measured counterclockwise from the receding (southern) major axis in the face-on image. Since $\theta=0^{\circ}$ to $180^{\circ}$ on the western side of the galaxy (which is the near side), equation (1) has a minus sign in front of $v_{\mathrm{r}}$ so that $v_{r}$ is positive for radial expansion.

As noted above, the isovelocity contours in the northern half of the galaxy, east of the major axis, do not show the upward curvature expected for rotation and, thus, the velocity field here is unlike that of the rest of the galaxy. Therefore the streaming motions in the northeast quadrant must differ from those in the rest of the galaxy. Relative to 


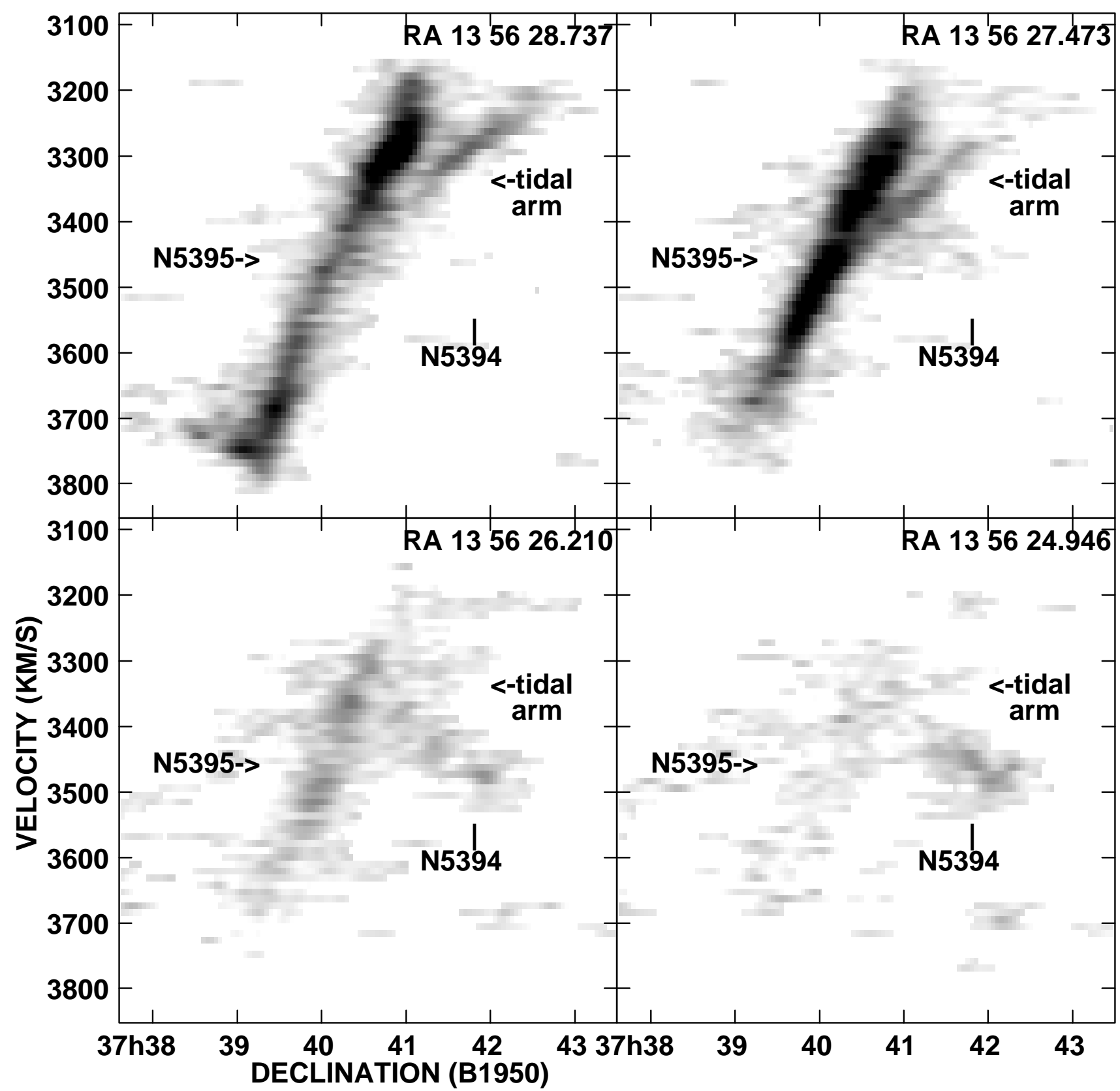

FIG. 14.-Gray-scale display of four declination-velocity diagrams from the $\mathrm{H}$ I cube, spaced at $15^{\prime \prime}$ intervals in right ascension, starting at the center of the NGC 5395 ring (top left panel) and ending at the center of NGC 5394 (bottom right panel). This demonstrates how the H I emission from NGC 5394 , the main disk of NGC 5395, and the northern tidal arm of NGC 5395 can be separated.

velocities on the northern (approaching) major axis, the observed values of $\left(v_{\text {obs }}-v_{\text {sys }}\right)$ east of the northern major axis are more negative than in the case of pure circular motion. From equation (1), one sees that the flatness of the $\mathrm{H}$ I velocity contours in the northeast quadrant could be accounted for with suitable nonuniform motions. The following examples would each work to flatten the velocity contours here: (1) if $\Delta \mathrm{v}_{t}=v_{z}=0$, then the radial motions of the gas must be inward in the northeast quadrant; (2) if $v_{r}=v_{z}=0$, then $\Delta v_{t}$ in the northeast quadrant must be greater than on the northern (approaching) major axis; (3) if $v_{r}=\Delta v_{t}=0$, then $v_{z}$ in the northeast quadrant must be smaller than on the northern major axis (e.g., the disk in the northeast quadrant could be folding toward us). The suggestion that the $\mathrm{H}$ I gas in the northeast quadrant is stream- ing radially inward is just opposite to the conclusion of $\S 5.1$ that the orientation of the $\mathrm{H} \alpha$ velocity kinks is in the sense of outward streaming motion of the ring here. Both processes could be occurring, i.e., the ring could be expanding outward whereas the $\mathrm{H}$ I gas east and northeast of the ring could be falling back in. One might expect this to produce a shock front, unless the two streams are not in the same plane. There is somewhat enhanced $\lambda 20 \mathrm{~cm}$ continuum emission from the northeast side of the ring, but the excess can be attributed to the radio continuum source just south of caustic 1 . In the merger remnants NGC 7252 and NGC 3921, Hibbard \& van Gorkom (1996b) and Hibbard \& Mihos (1996a) find that the $\mathrm{H}$ I in the tidal arm near the disk has started falling back in. A similar situation may be occurring in the northeast quadrant of NGC 5395. 


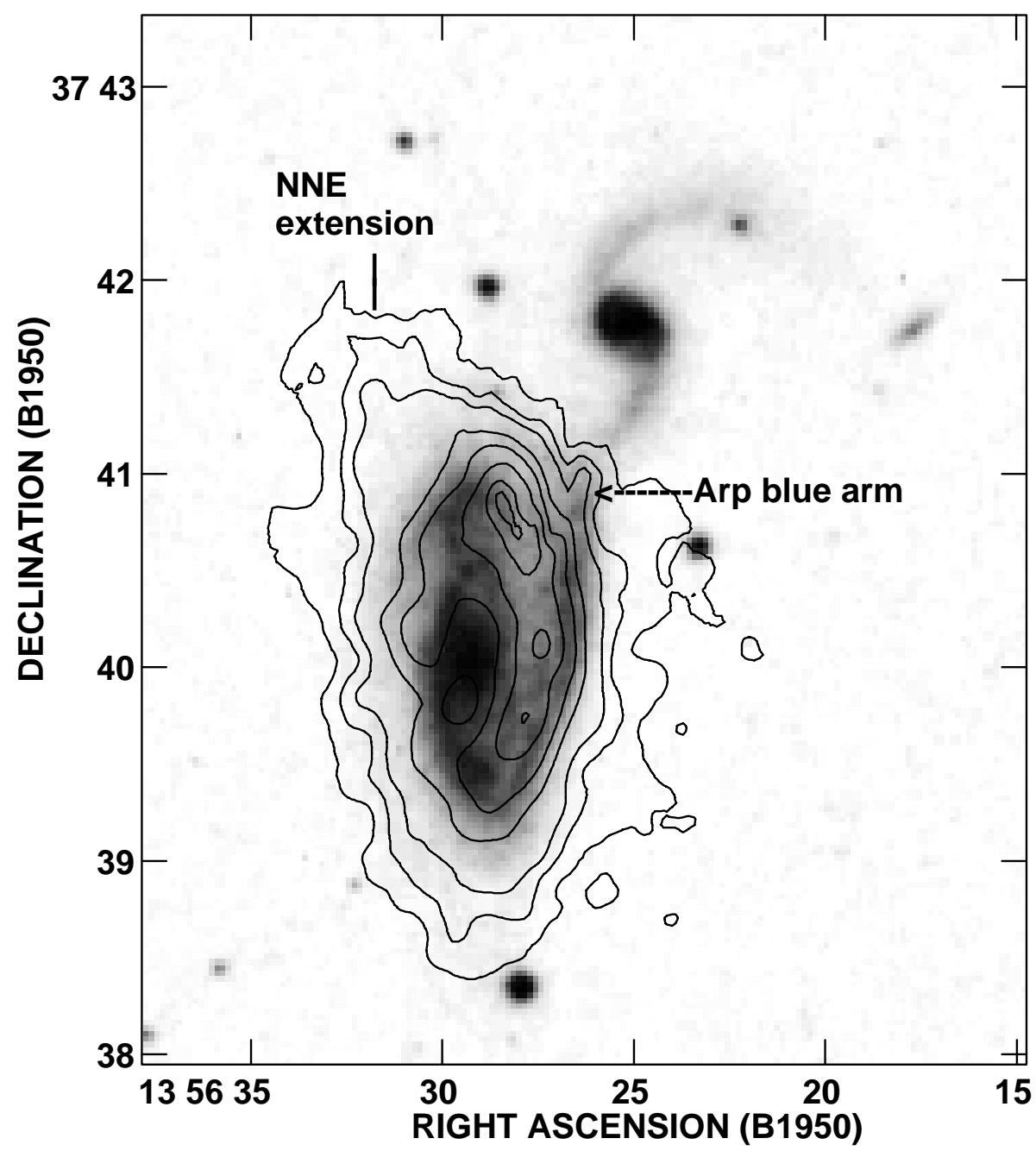

FIG. 15.-Gray-scale display of the Digitized Sky Survey image overlaid with contours from the low-resolution $N(\mathrm{H}$ I $)$ image of the disk of NGC 5395 after removal of the northern $\mathrm{H}$ I tidal arm with the peculiar velocity. The line-of-sight column density contour levels are at 3.2, 6.8, 10, 19, 26, 34, 41, $44 \times 10^{20}$ atoms cm ${ }^{-2}$.

\subsubsection{H I Velocity Dispersion}

IC 2163, NGC 2207, NGC 2535, and NGC 5774/75 (Elmegreen et al. 1995a; Kaufman et al. 1997; Irwin 1994) have a widespread, high-velocity dispersion in the $\mathrm{H}$ I gas. Elmegreen et al. (1993) suggested that close encounters increase the gas turbulence and the gravitational Jeans mass of the largest clouds. We corrected the $\mathrm{H}$ I second moment image of the NGC 5395 disk for the velocity gradient across the beam but not for systematic velocity gradients along line of sight. The resulting $\mathrm{H}$ I velocity dispersion image has values of $50-70 \mathrm{~km} \mathrm{~s}^{-1}$ over much of the disk (see Fig. 23). These very high values for the onedimensional velocity dispersion are likely to contain major contributions from the following sources. (1) The line of sight intercepts gas at various radial distances and, thus, various velocities (see Irwin \& Seaquist 1991; Irwin 1994). This is important because of the relatively high inclination. (2) The line of sight intercepts gas at various altitudes above the plane. This can make an important contribution to the observed velocity dispersion because of the threedimensional disturbed structures in this galaxy. Because of the complications introduced by noncircular motions and nonplanar features, one needs a detailed modeling of the effects of the encounter to decide if there is, indeed, wide- spread, high gas turbulence in NGC 5395. Although in $\S 9$, we present a galaxy encounter simulation that reproduces some of the main features of this system, the detailed modeling required to determine the gas turbulence is beyond the scope of this paper.

\subsection{Northern H I Tidal Arm of NGC 5395}

The northern $\mathrm{H}$ I tidal arm of NGC 5395 has a projected length of $28 \mathrm{kpc}$. It lies to the east of NGC 5394 and extends past it to the north. The tidal arm is an extension of the dominant spiral arm ( $m=1$ mode) seen in the optical disk. North of the ring in Figure 1, there is a faint optical feature closely parallel to part of the northern $\mathrm{H}$ I tidal arm (see Figs. 16 and 17). Because gas suffers collisions and stars do not, the optical arm need not coincide with the $\mathrm{H}$ I arm. The northern $\mathrm{H}$ I tidal arm is not the continuation of the Arp blue arm; the southern part of the $\mathrm{H}$ I tidal arm starts $11^{\prime \prime}-16^{\prime \prime}$ east of the Arp blue arm and heads north-northeast whereas the Arp blue arm heads nearly due north. The point where the northern $\mathrm{H}$ I tidal arm overlaps the disk in velocity is marked by an " $x$ " in Figure 20. There is a bright $\mathrm{H}$ II region at this location, which may be a coincidence. The northern $\mathrm{H}$ I tidal arm may continue farther south at the same velocity as the disk, or the tidal arm and the spiral 


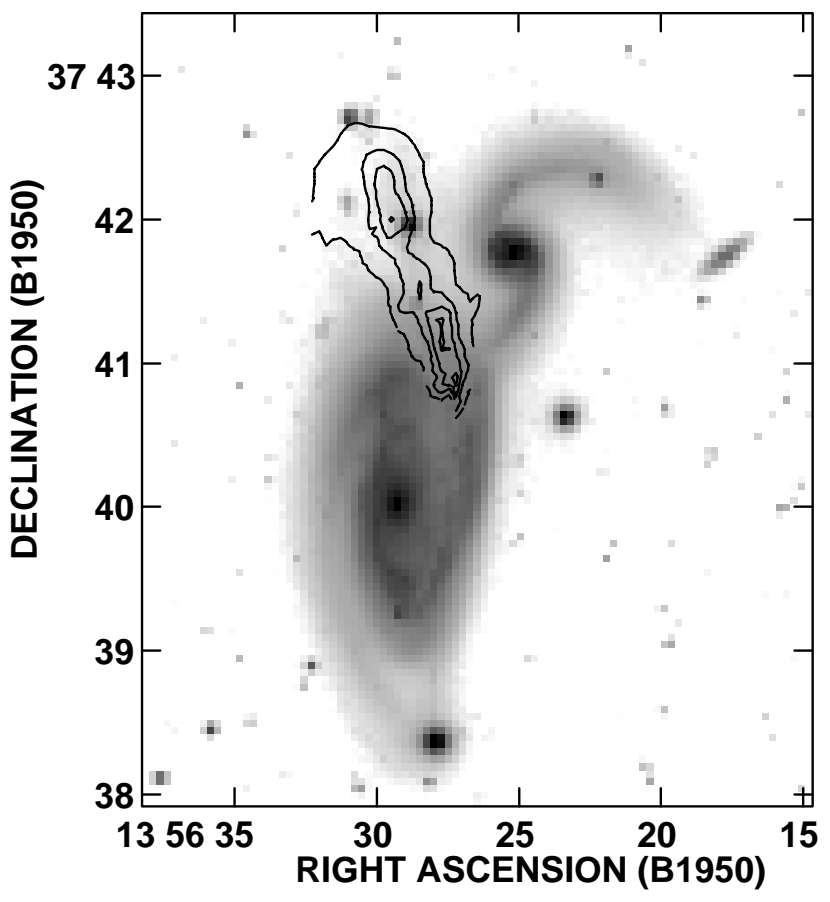

FIG. 16.-Gray-scale display of the $r$-band image from the ChengalurNordgren galaxy survey on a logarithmic intensity scale overlaid with contours from the low-resolution $N(\mathrm{H}$ I) image of the northern $\mathrm{H}$ I tidal arm of NGC 5395. The line-of-sight column density contour levels are at $3.2,6.8,8.5,10 \times 10^{20}$ atoms $\mathrm{cm}^{-2}$.

arm may overlap, forming a caustic knot (i.e., the intersection of discrete orbit streams), but this is difficult to tell from our viewing angle. One expects radio continuum emission from the shock front at a caustic knot. Although the point

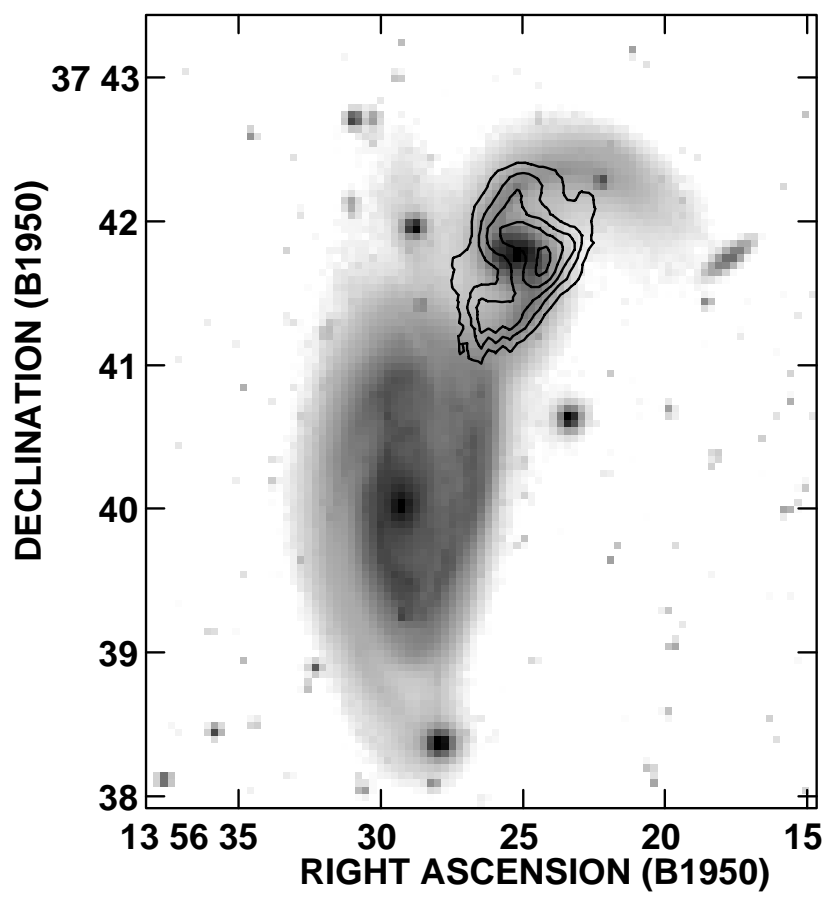

FIG. 17.-Gray-scale display of the $r$-band image from the ChengalurNordgren galaxy survey on a logarithmic intensity scale overlaid with contours from the low-resolution $N(\mathrm{H} \mathrm{I})$ image of NGC 5394. The line-ofsight column density contour levels are at $2.0,3.4,4.8,6.1,7.5 \times 10^{20}$ atoms $\mathrm{cm}^{-2}$.

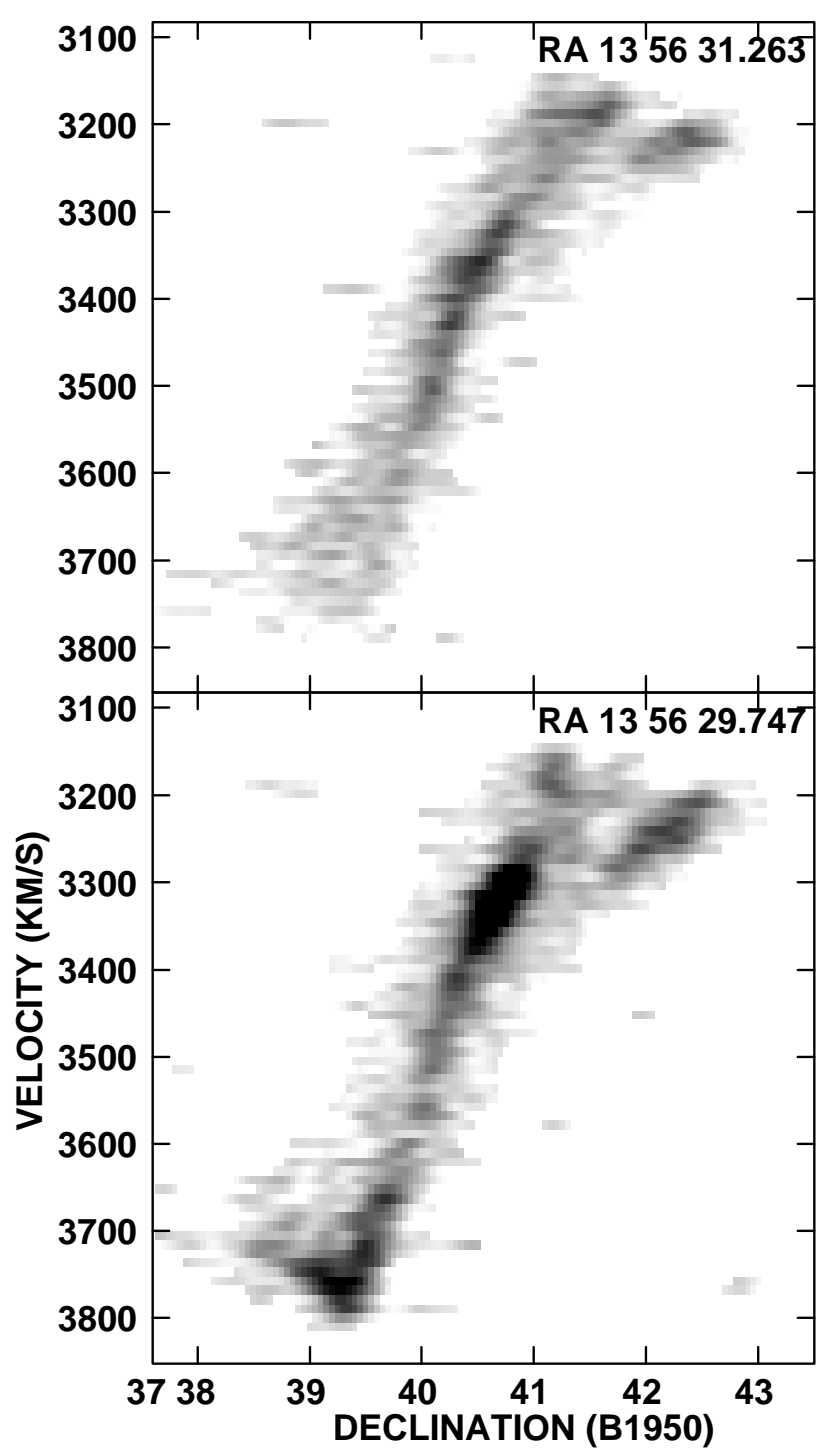

FIG. 18. - Gray-scale display of two $\mathrm{H}$ I declination-velocity diagrams of the eastern side of NGC 5395. The bottom panel includes the eastern ridge of caustic 1 in NGC 5395, and the top panel is $18^{\prime \prime}$ farther east. Three distinct velocity features can be seen in the northern part of NGC 5395; the long northern $\mathrm{H} \mathrm{I}$ tidal arm is the feature extending farthest to the north, the north-northeast extension is the middle feature (the one that ends at declination $=37^{\circ} 42^{\prime} 00^{\prime \prime}$ ), and the main disk is the feature ending at declination $=37^{\circ} 41^{\prime} 15^{\prime \prime}$ in the bottom panel.

" $\mathrm{x}$ " is located in a region of $\lambda 20 \mathrm{~cm}$ continuum emission on the ring, the closest radio continuum peak is $10^{\prime \prime}$ east of the $\mathrm{H}$ II region (see Fig. 5).

The $\mathrm{H}$ I velocity field of the northern $\mathrm{H}$ I tidal arm is displayed as isovelocity contours in Figure 24 overlaid on the $N(\mathrm{H} \mathrm{I})$ image of the tidal arm in gray scale. The line-ofsight velocity of the northern $\mathrm{H}$ i tidal arm is $75-100 \mathrm{~km} \mathrm{~s}^{-1}$ greater than the line-of-sight velocity of the disk at the same projected location (near the southern end of the northern $\mathrm{H}$ I tidal arm but north of the region that could be contaminated by NGC 5394; see Fig. 16). Since this is the approaching side of the galaxy, it means that $\left(v_{\text {obs }}-v_{\text {sys }}\right)$ is less negative on the northern $\mathrm{H}$ I tidal arm than in the disk. The velocity difference could result from a combination of outward streaming motions along the tidal arm, a decrease 


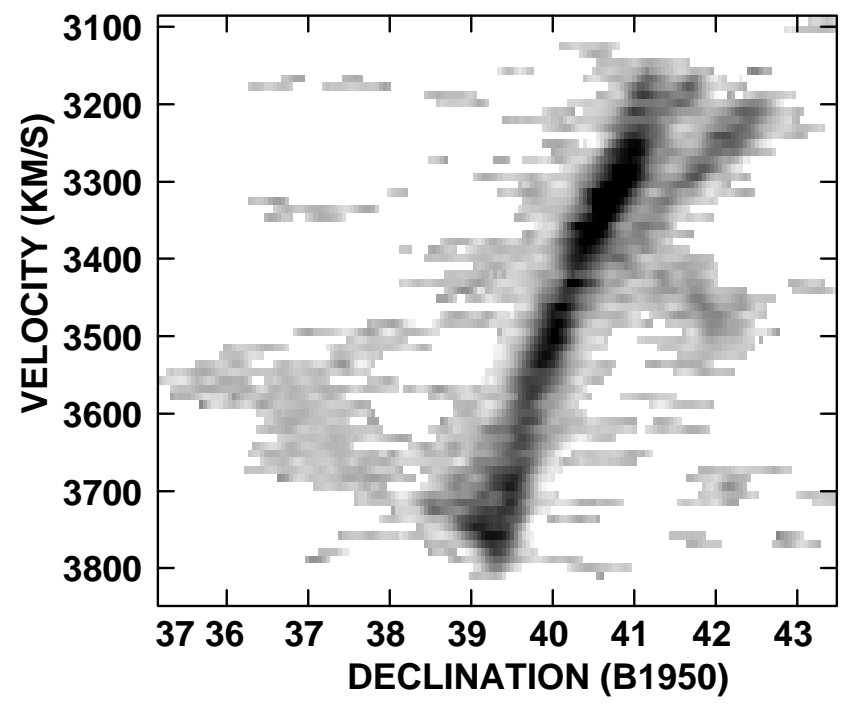

FIG. 19.- Gray-scale display of an $\mathrm{H}$ I declination-velocity diagram in which the emission is summed over all the values of R.A. relevant to the system. This shows that the broad, diffuse southern tidal arm of NGC 5395 connects in velocity to the southern end of the main disk of NGC 5395. in sin $i$ on the tidal arm as compared to the main disk, and the effect of the sky-plane projection on the apparent relative locations of features not in the same plane. If the velocity difference results solely from a change in $\sin i$, with no streaming, then taking the disk inclination as $65^{\circ}$ and using measured values for the line-of-sight velocities of the northern $\mathrm{H}$ I tidal arm and the disk, we find that the tidal arm would need to have an inclination of $20^{\circ}-25^{\circ}$ with respect to the sky plane. Large streaming motions along the line of sight would be required to produce the $40^{\circ}-45^{\circ}$ change in inclination, and these would contribute to the measured velocity difference between the tidal arm and the disk. If the streaming motions are away from us, then the required change in inclination is less than $40^{\circ}$. The velocity gradient along the tidal arm is about half the velocity gradient farther south in the main disk; this could result from streaming motions in the tidal arm.

\subsection{NGC 5394}

Figure 25 displays the $\mathrm{H}$ I isovelocity contours of NGC 5394 overlaid on the $N(\mathrm{H} \mathrm{I})$ image of NGC 5394 in gray scale, both from the low-resolution data. The plus sign marks the position of the $\mathrm{H} \alpha$ nucleus, and the triangle, the

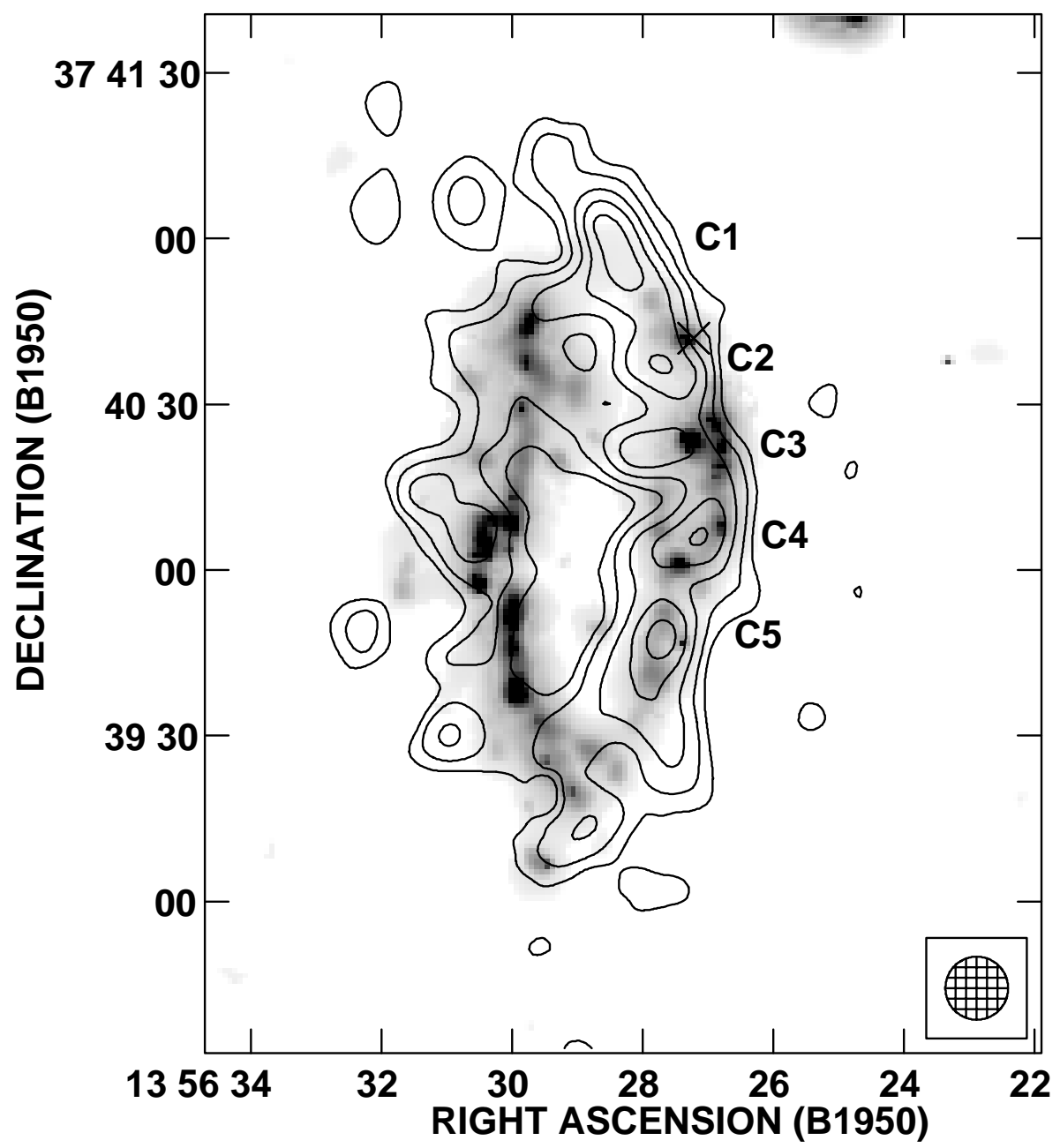

FIG. 20.-Gray-scale display of the composite-resolution $\mathrm{H} \alpha$ surface brightness image overlaid with contours from the high-resolution $N(\mathrm{H}$ I $)$ image of the disk of NGC 5395 after removal of the northern $\mathrm{H}$ I tidal arm. Five massive $\mathrm{H}$ I clouds, each with $\mathrm{H}$ I mass in excess of $10^{8} M_{\odot}$, are labelled. The times sign marks where the northern $\mathrm{H}$ I tidal arm overlaps the disk in velocity. The line-of-sight column density contour levels are at $10,17,26,34$, and $43 \times 10^{20}$ atoms $\mathrm{cm}^{-2}$. The beam symbol represents the $\mathrm{H}$ I resolution. 


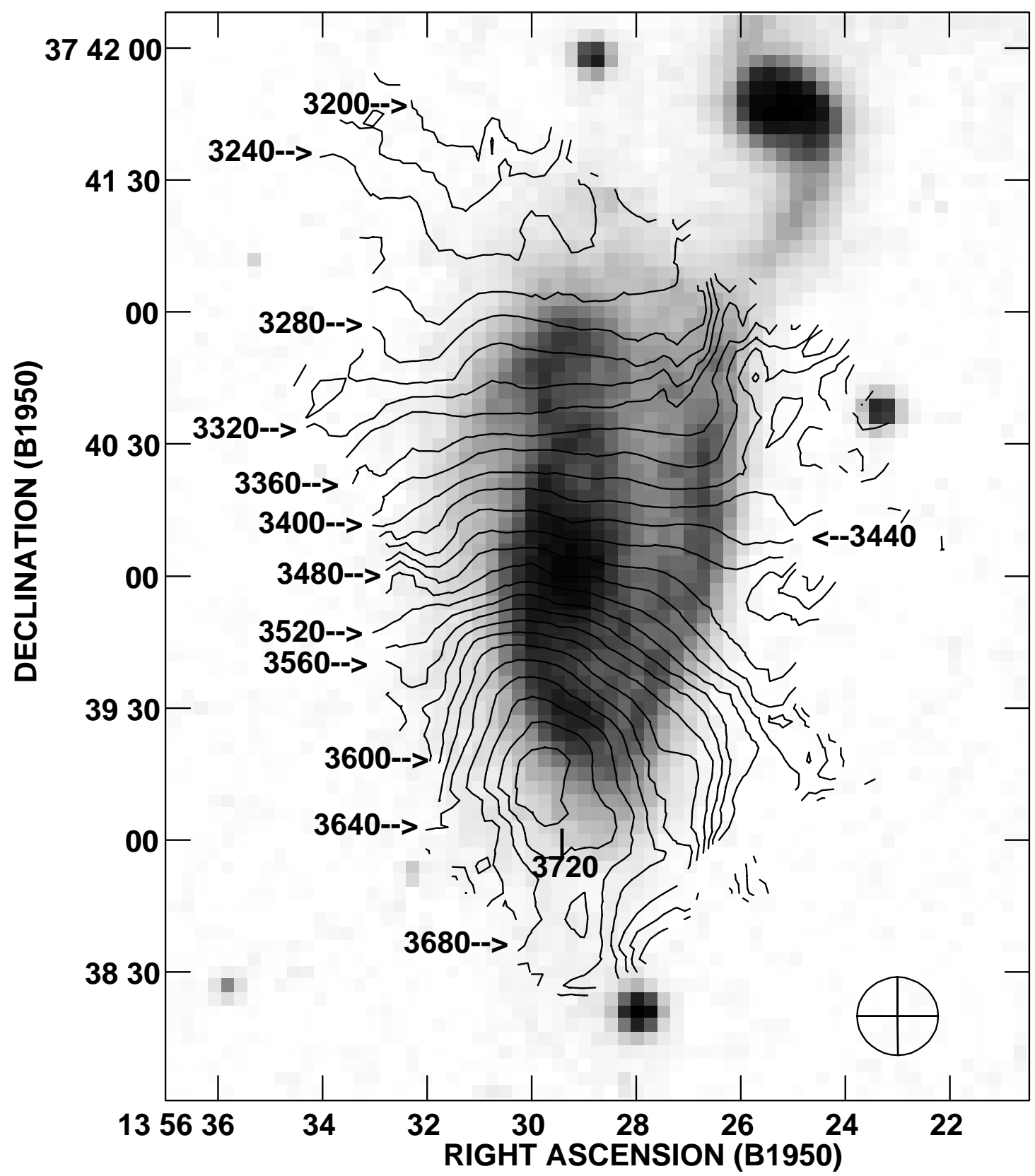

FIG. 21.-Gray-scale display of the Digitized Sky Survey image overlaid with H I isovelocity contours from the low-resolution cube of the disk of NGC 5395 after removal of the northern $\mathrm{H}$ I tidal arm with the peculiar velocity. The contour interval is $20 \mathrm{~km} \mathrm{~s}^{-1}$. The beam symbol represents the $\mathrm{H}$ I resolution.

position of the radio continuum peak. The strongest $\mathrm{H}$ I emission is from the inner part of the southern tidal arm and possibly the inner spiral arms west of the nucleus.

The H I velocity contour through the nucleus has $\mathrm{v}=3464 \mathrm{~km} \mathrm{~s}^{-1}$. This is only marginally higher than Karachentsev's (1980) value of $3451 \pm 12 \mathrm{~km} \mathrm{~s}^{-1}$. The H I kinematic minor axis has position angle $=90^{\circ}$ and $v=3470 \pm 5 \mathrm{~km} \mathrm{~s}^{-1}$. We take $v_{\text {sys }}(\mathrm{H} \mathrm{I})$ as $3465 \pm 5 \mathrm{~km} \mathrm{~s}^{-1}$. Thus, from the $\mathrm{H}$ I velocity fields, the systemic velocity of NGC 5394 is $15 \pm 11 \mathrm{~km} \mathrm{~s}^{-1}$ greater than the systemic velocity of NGC 5395. The kinematic major axis of NGC 5394 twists because of streaming motions on the tidal arms. Since the near side is the western side, the sense of the twist of the velocity contours at the arms is consistent with streaming outward. This means that the outer tidal arms are outside corotation.
If the line of nodes of the projection is taken perpendicular to the kinematic minor axis, then it has a position angle of $0^{\circ}$. This differs from the $\approx 60^{\circ}$ position angle of the photometric major axis at the end of the inner spiral arms (see Fig. 6). The photometric major axis does not drop to $0^{\circ}$ until $r=40^{\prime \prime}$. Hence, there is a large misalignment between the photometric and kinematic axes inside $40^{\prime \prime}$. Elmegreen et al. (1995a) and Kaufman et al. (1997) find a significant misalignment between the photometric and kinematic axes in the ocular galaxies IC 2163 and NGC 2535, respectively, and they use it to argue for an intrinsically oval shape to the central disk. It is likely that the same explanation applies to NGC 5394. The encounter simulation model in $\S 9$ below finds that the disk of NGC 5394 is presently elongated.

Along the line of nodes of the projection, at a position angle of $0^{\circ},\left(v_{\max }\right) \sin i$ is $20 \mathrm{~km} \mathrm{~s}^{-1}$ for $\mathrm{H} \mathrm{I}$ and $27 \mathrm{~km} \mathrm{~s}^{-1}$ 


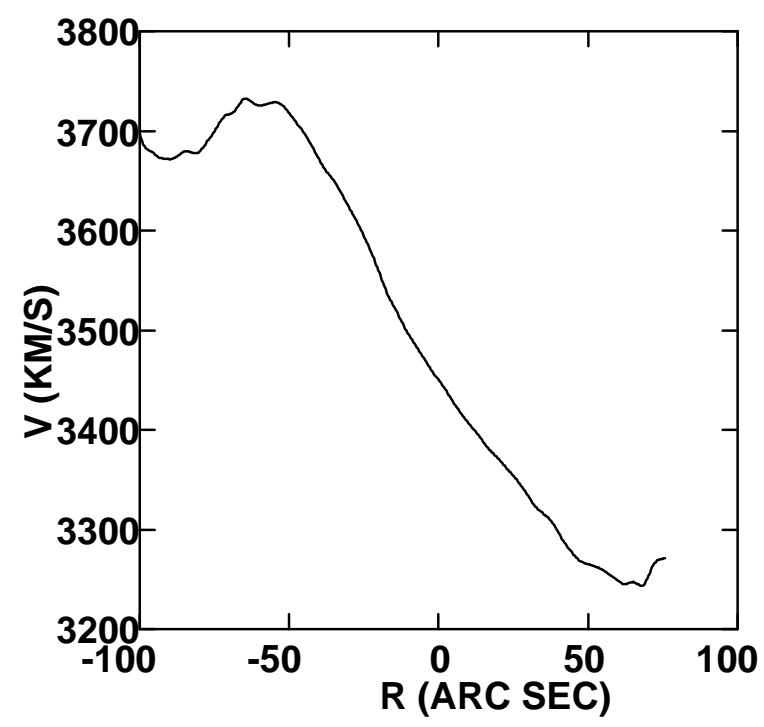

FIG. 22.-H I velocity vs. position in the disk of NGC 5395 for a slice along the kinematic major axis with distance measured from the center of the ring. The south side is on the left (negative positions), and the north side is on the right. The values of $v \sin i$ in this diagram are evidence for noncircular motions on the kinematic major axis.

for $\mathrm{H} \alpha$. In $\S 6.3 .2$, we estimated from the Persic-Salucci relation that an undisturbed spiral galaxy with the same luminosity as NGC 5395 would have $v_{\max }=235 \pm 33 \mathrm{~km}$ $\mathrm{s}^{-1}$. If we use the ratio of $I$-band luminosities $(0.23 \pm 0.02$ after subtracting the starburst) for these two galaxies to get the line width of NGC 5394, we should multiply the value

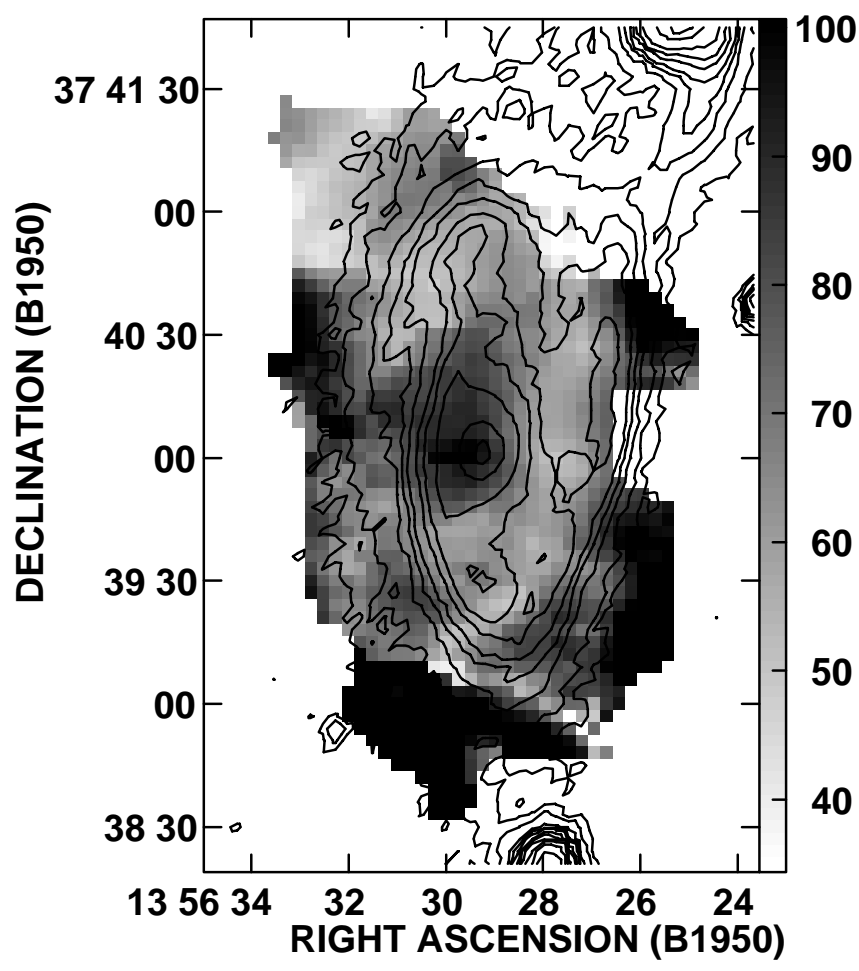

FIG. 23.-Gray-scale display of the $\mathrm{H}$ I second-moment image of the disk of NGC 5395 after correction for velocity gradients across the beam. This is overlaid with $R$-band contours. The labels on the wedge refer to the velocity dispersion in $\mathrm{km} \mathrm{s}^{-1}$. The high values for the $\mathrm{H}$ I velocity dispersion are likely to result, in part, because the line of sight intercepts gas at various radial distances and at various altitudes above the plane.

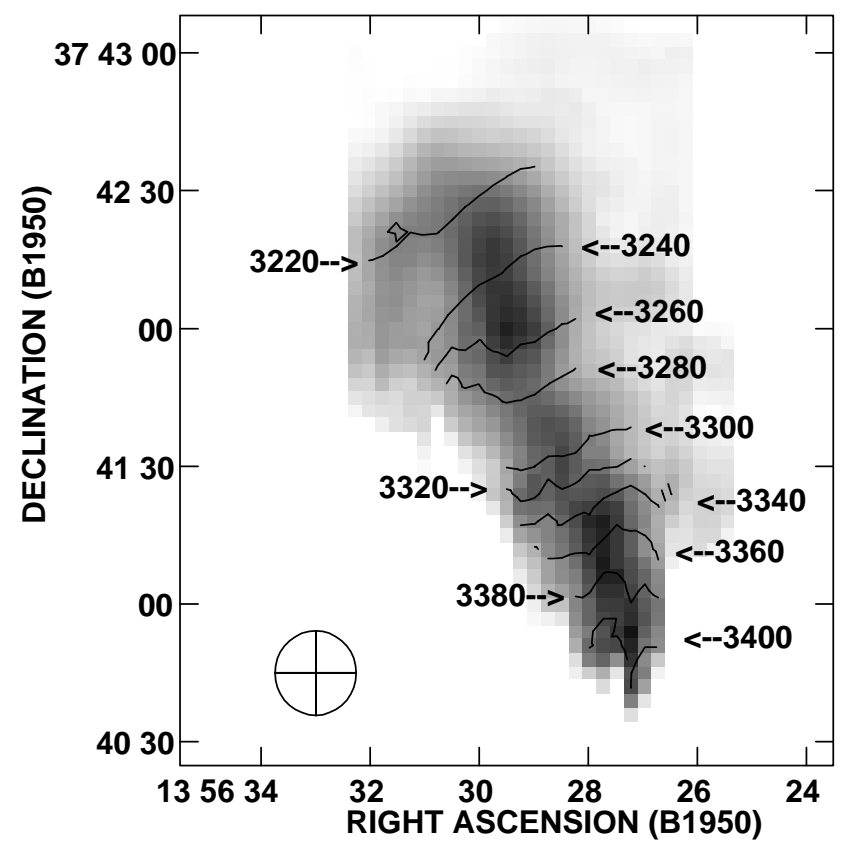

FIG. 24.- $\mathrm{H}$ I isovelocity contours overlaid on $N(\mathrm{H} \mathrm{I})$ in gray scale for the northern H I tidal arm of NGC 5395 (from the low-resolution data). The contour interval is $20 \mathrm{~km} \mathrm{~s}^{-1}$.

for NGC 5395 by $(0.23 \pm 0.02)^{1 / 4}$ to get $v_{\max }=163 \pm 26 \mathrm{~km}$ $\mathrm{s}^{-1}$ in NGC 5394. When compared with the observed value of $\left(v_{\max }\right) \sin i$ of $27 \mathrm{~km} \mathrm{~s}^{-1}$ in NGC 5394, this implies $i$ in the range $8^{\circ}$ to $11^{\circ}$. Thus NGC 5394 is nearly face-on.

Why does the inner spiral arm east of the nucleus show no evidence of ongoing star formation in the optical images? Kennicutt (1989) found that for star-forming

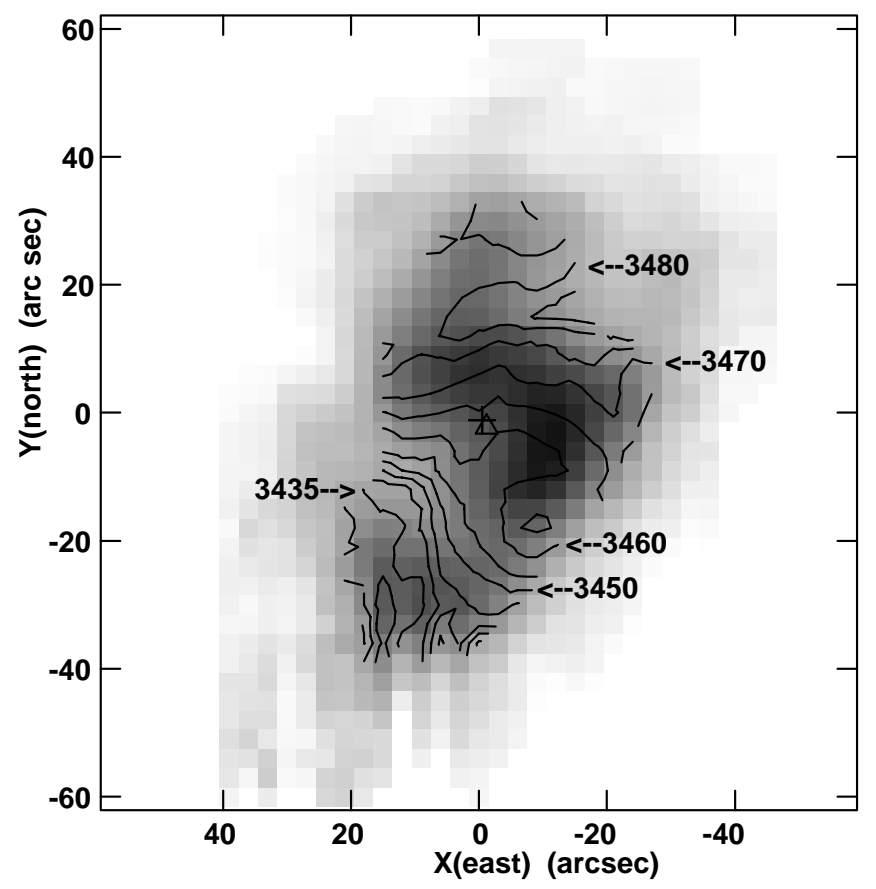

FIG. 25.- $\mathrm{H}$ I isovelocity contours overlaid on $N(\mathrm{H} \mathrm{I})$ in gray scale for NGC 5394, from the low-resolution $\left(18^{\prime \prime} .4 \times 17^{\prime \prime} .7\right)$ data. The contour interval is $5 \mathrm{~km} \mathrm{~s}^{-1}$. The plus sign marks the position of the $\mathrm{H} \alpha$ nucleus, and the triangle the radio continuum position of the nucleus from the FIRST survey. The triangle partially overlaps the plus sign. 


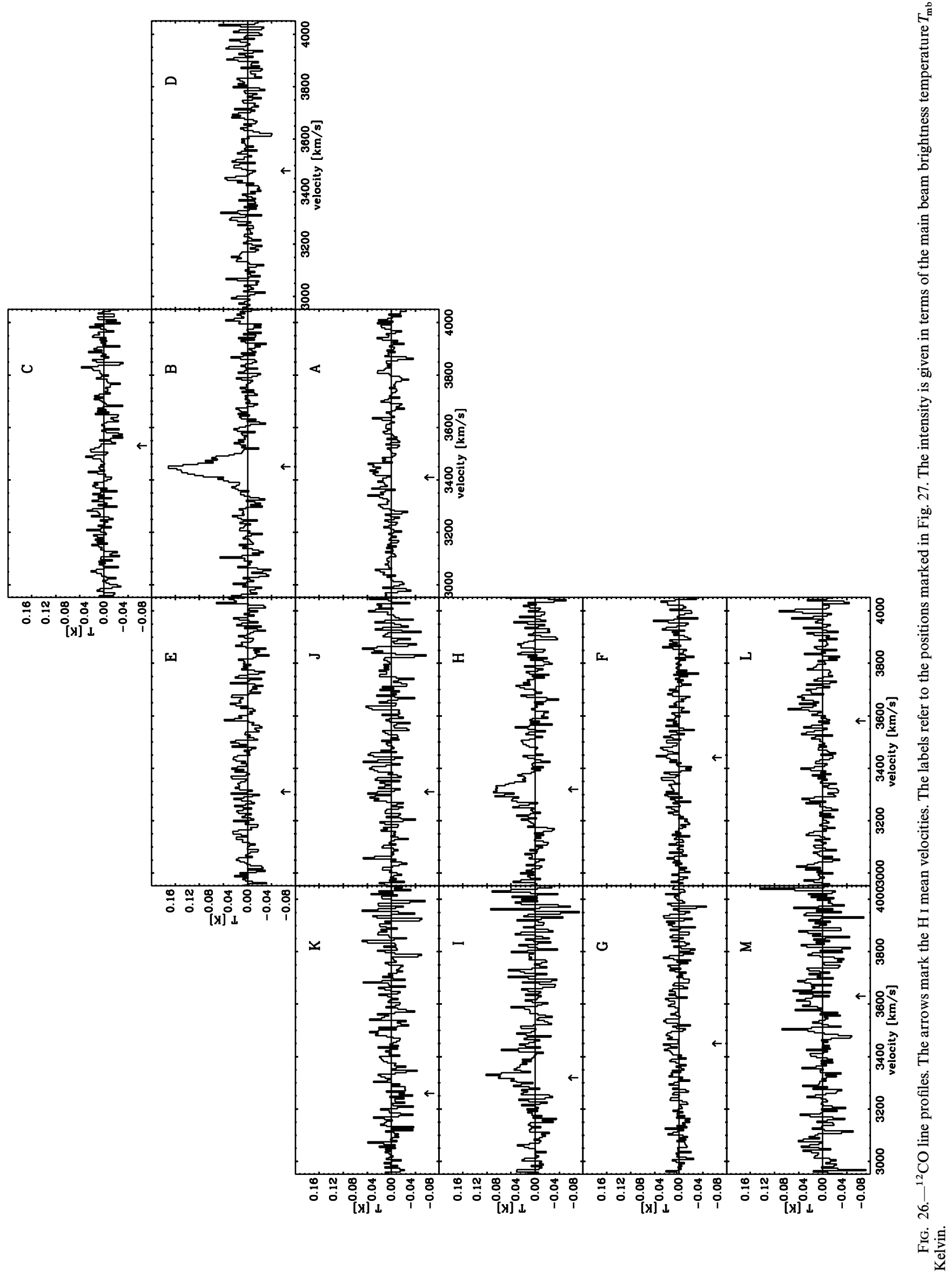


regions in late-type spirals, the instability parameter $Q_{\text {gas }}=$ $\kappa \sigma_{v, g} / \pi G \mu_{\text {gas }} \leq 1.4$, where $\kappa$ is the epicyclic frequency, $\sigma_{v, g}$ is the one-dimensional $\mathrm{H}$ I velocity dispersion, and $\mu_{\mathrm{gas}}$ is the face-on surface density of gas. In the high-resolution (11".4 FWHM) $N(\mathrm{H} \mathrm{I})$ image, the $\mathrm{H}$ I column density on this arm is $\sim 7 \times 10^{20}$ atoms $\mathrm{cm}^{-2}$. This is representative of the average column density over the interstellar Jeans length and is thus relevant for $Q_{\text {gas }}$. There could also be molecular gas in the arms; our ${ }^{12} \mathrm{CO}$ observations (see $\S 7$ below) do not have sufficient spatial resolution to distinguish between emission from the nucleus region and the arm. The correction to $\mu_{\text {gas }}$ for projection is small. Thus we take $\mu_{\text {gas }} \approx 7$ $\times 10^{20}$ atoms cm ${ }^{-2}$ in the arm, with a possible increase if the $\mathrm{H}_{2}$ surface density is important. After correcting for the average velocity gradient, $\sigma_{v, g}$ in much of the disk is large, $20-40 \mathrm{~km} \mathrm{~s}^{-1}$, as compared to values of $6-13 \mathrm{~km} \mathrm{~s}^{-1}$ in undisturbed spirals (see references in Kaufman et al. 1997). At the eastern inner arm, $\sigma_{v, g}=30 \mathrm{~km} \mathrm{~s}^{-1}$. The epicyclic frequency $\kappa=2 v_{c} / R$ because the rotation curve is rising. At the radial distance of the $\operatorname{arm}\left(R=10^{\prime \prime}=2.3 \mathrm{kpc}\right)$, and with $i=9^{\circ}$, one has $v_{c}=42 \mathrm{~km} \mathrm{~s}^{-1}$, so $\kappa=3.7 \mathrm{~km} \mathrm{~s}^{-1} \mathrm{kpc}^{-1}$. These values give $Q_{\mathrm{gas}} \approx 15$, if the molecular surface density is small, or $Q_{\text {gas }}<15$, if molecular gas is important. Thus it is possible that $Q_{\text {gas }}$ is too high for significant star formation in the eastern inner arm.

The large values of $\sigma_{v, g}$ in much of the disk of NGC 5394 are similar to those in NGC 2535, IC 2163, NGC 2207, and NGC 5774/75 (Kaufman et al. 1997; Elmegreen et al. 1995a; Irwin 1994). This implies large scale heights for the gas in NGC 5394 (see discussion of scale heights in Kaufman et al. 1997).

\section{CO DETECTIONS}

Figure 26 displays the line profiles at 13 positions where we searched for ${ }^{12} \mathrm{CO}$ emission. The arrow in each profile indicates the mean $\mathrm{H}$ I velocity. The positions are distributed on a grid with one beam (33") spacing; they are marked on the gray-scale display of the Digitized Sky Survey image in Figure 27. We also observed four positions (BA, BC, BD, and BE) halfway between position $\mathrm{B}$ and positions $\mathrm{A}, \mathrm{C}, \mathrm{D}$, and $\mathrm{E}$, respectively.

There are three clear detections in Figure 26: position B in NGC 5394 and positions $\mathrm{H}$ and I in the northern half of NGC 5395. There are also three possible detections in posi- tions A (NGC 5394) and L and M (NGC 5395). Table 6 lists the values of the rms noise level and the peak $T_{\mathrm{mb}}$, and the values calculated from Gaussian fits to the line profiles of the amplitude, the center velocity $v$, the integrated intensity $I_{\mathrm{CO}}=\int T_{\mathrm{mb}} d v$, and the $\mathrm{H}_{2}$ mass. For the latter, we used the conversion factor $N\left(\mathrm{H}_{2}\right)=2.3 \times 10^{20} I_{\mathrm{CO}} \mathrm{H}_{2} \mathrm{~cm}^{-2}$ from Strong et al. (1988). Calculating $I_{\mathrm{CO}}$ by direct integration of the spectrum gives values very similar to those obtained from the Gaussian fits, but this method is somewhat sensitive to the choice of integration limits when the signal-tonoise level is not high.

We also have clear detections of $\mathrm{CO}$ emission at the intermediate positions $\mathrm{BA}, \mathrm{BC}$, and $\mathrm{BD}$, but these intermediate positions had pointing problems. The similar line profiles and the values for $I_{\mathrm{CO}}$ (about the same integrated intensity at position $\mathrm{BD}$ as at position $\mathrm{B} ; 20 \%$ to $40 \%$ less at positions $\mathrm{BC}$ and $\mathrm{BA}$, respectively) suggest that the emission at these intermediate positions is from the central source. This is consistent with the absence of or very weak detections at positions $\mathrm{A}, \mathrm{C}$, and $\mathrm{D}$. Although there may be some $\mathrm{CO}$ emission from the western inner arm included at positions B and $\mathrm{BD}$, we cannot make a strong statement about this because of the pointing problems for the intermediate positions.

Positions $\mathrm{H}, \mathrm{F}$, and $\mathrm{L}$ are well-centered on the main dust lane on the west arm of NGC 5395. Note that there is more $\mathrm{CO}$ emission from the northern part than from the southern part of NGC 5395. Position $\mathrm{H}$, which has the highest value of $I_{\mathrm{CO}}$ in NGC 5395, contains the strongest $\mathrm{H}$ I emission (see Fig. 15). Usually, the strongest $\mathrm{CO}$ emission comes from the central part of a galaxy, but this is not the case in NGC 5395, as positions $F$ and $\mathrm{G}$ are closer to the nucleus than positions $\mathrm{H}$ and $\mathrm{I}$. Less than $20 \%$ of the gas detected in NGC 5395 is in molecular form, and the $\mathrm{H}$ I trough in the center of NGC 5395 is not filled in with CO emission. Thus the ring is a ring in total gas as well as in $\mathrm{H} \mathrm{I}$.

In NGC 5394, there is a lot of molecular gas in the aperture centered on the starburst nucleus, $3.5 \times 10^{9} M_{\odot}$ (including a $40 \%$ by mass contribution from helium). The molecular mass is four times the $\mathrm{H}$ I mass of the galaxy. It appears that most of the gas presently associated with NGC 5394 has fallen into this central (3.8 kpc radius) region as a result of the encounter and is now in molecular form, suitable for fueling the starburst. From the $\mathrm{H} \mathrm{I}$ and $\mathrm{CO}$ lumi-

TABLE 6

\begin{tabular}{lcccccc}
\multicolumn{7}{c}{${ }^{12}$ CO MEASUREMENTS } \\
\hline \hline Position & $\begin{array}{c}\text { Noise } \\
(\mathrm{K})\end{array}$ & $\begin{array}{c}\text { Peak } T_{\mathrm{mb}} \\
(\mathrm{K})\end{array}$ & $\begin{array}{c}\text { Amplitude } \\
(\mathrm{K})\end{array}$ & $\begin{array}{c}v \\
\left(\mathrm{~km} \mathrm{~s}^{-1}\right)\end{array}$ & $\begin{array}{c}I_{\mathrm{CO}} \\
\left(\mathrm{K} \mathrm{km} \mathrm{s}^{-1}\right)\end{array}$ & $\begin{array}{c}M\left(\mathrm{H}_{2}\right) \\
\left(10^{9} M_{\odot}\right)\end{array}$ \\
\hline $\mathrm{A} \ldots \ldots$. & 0.016 & 0.051 & 0.029 & 3424 & 3.3 & 0.79 \\
$\mathrm{~B} \ldots \ldots$. & 0.019 & 0.165 & 0.147 & 3443 & 10.9 & 2.6 \\
$\mathrm{C} \ldots \ldots$. & 0.017 & $\ldots$ & $\ldots$ & $\ldots$ & $\ldots$ & $\ldots$ \\
$\mathrm{D} \ldots \ldots$. & 0.018 & $\ldots$ & $\ldots$ & $\ldots$ & $\ldots$ & $\ldots$ \\
$\mathrm{E} \ldots \ldots$. & 0.018 & $\ldots$ & $\ldots$ & $\ldots$ & $\ldots$ & $\ldots$ \\
$\mathrm{F} \ldots \ldots$. & 0.014 & $\ldots$ & $\ldots$ & $\ldots$ & $\ldots$ & $\ldots$ \\
$\mathrm{G} \ldots \ldots$. & 0.016 & $\ldots$ & $\ldots$ & $\ldots$ & $\ldots$ & $\ldots$ \\
$\mathrm{H} \ldots \ldots$. & 0.014 & 0.087 & 0.076 & 3314 & 6.2 & 1.5 \\
$\mathrm{I} \ldots \ldots \ldots$ & 0.017 & 0.102 & 0.061 & 3328 & 4.5 & 1.1 \\
$\mathrm{~J} \ldots \ldots \ldots$ & 0.018 & $\ldots$ & $\ldots$ & $\ldots$ & $\ldots$ & $\ldots$ \\
$\mathrm{K} \ldots \ldots$. & 0.016 & $\ldots$ & $\ldots$ & $\ldots$ & $\ldots$ & $\ldots$ \\
$\mathrm{L} \ldots \ldots$. & 0.018 & 0.072 & 0.037 & 3653 & 3.0 & 0.72 \\
$\mathrm{M} \ldots \ldots$. & 0.023 & 0.062 & 0.029 & 3637 & 3.1 & 0.72 \\
\hline
\end{tabular}


nosities, the total mass of gas (including helium) is $4.5 \times 10^{9}$ $M_{\odot}$ for NGC 5394 and $2.3 \times 10^{10} M_{\odot}$ for NGC 5395. This gives an NGC 5394/95 mass ratio in total gas of 0.2, comparable to the $I$-band luminosity ratio of 0.25 .

The value of $I_{\mathrm{CO}}$ in the central 33" aperture on NGC 5394 corresponds to an average $N\left(\mathrm{H}_{2}\right)$ of $2.5 \times 10^{21} \mathrm{H}_{2} \mathrm{~cm}^{-2}$. If we adopt the standard Galactic value, $A_{v} / N_{\text {gas }}=0.53$ $\times 10^{-21} \mathrm{mag}$ per atom $\mathrm{cm}^{-2}$, from Bohlin, Savage, \& Drake (1978), this corresponds to an $A_{v}$ of $1.3 \mathrm{mag}$ to the midplane. If the molecular gas is strongly concentrated in the central $5^{\prime \prime}(=1.2 \mathrm{kpc})$ region, then $N\left(\mathrm{H}_{2}\right)$ in the nucleus region would be much higher than this average value. Thus it seems possible that extinction accounts for much of the difference (see $\S 3.2$ ) between the measured value of $S_{v}(20)$ for the nucleus region of NGC 5394 and that deduced from the uncorrected $\mathrm{H} \alpha$ flux. We detect no $\mathrm{CO}$ from the northern spiral arm of NGC 5394 and have a marginal detection on its southern spiral arm.

\section{DISCUSSION}

The main features of this system are the following. (1) NGC 5394 has two long, open tidal arms with high arminterarm contrast, a starburst in the nucleus plus some evidence for outflow, and three very bright inner spiral arms. The inner spiral arms are mostly smooth, and only one is clearly detected in $\mathrm{H} \alpha$ emission. (2) NGC 5395 has a long, narrow, $\mathrm{H}$ I tidal arm in the north and a broad, diffuse $\mathrm{H}$ I tidal arm in the south. The northern $\mathrm{H}$ I tidal arm is distinct in velocity from the main disk and has a line-of-sight velocity as much as $75-100 \mathrm{~km} \mathrm{~s}^{-1}$ greater than the main disk at the same projected location. (3) In NGC 5395, the encounter appears to be exciting $m=1$ and $m=0$ modes in what had been a two-armed spiral. NGC 5395 has a large ring of $\mathrm{H} \alpha$, radio continuum, and $\mathrm{H}_{\mathrm{I}}$ emission, somewhat off center relative to the nucleus. The eastern side of the ring is brighter in radio continuum and $\mathrm{H} \alpha$, whereas the western side is brighter in $\mathrm{H}$ I. The dynamical center of NGC 5395 has moved from the optical nucleus to the center of the ring. (4) The velocity field of NGC 5395 does not have large-scale reflection symmetry about the kinematic minor axis: there is a north-south asymmetry in velocities along the major axis, and the $\mathrm{H}$ I velocity field in the northeast quadrant of the galaxy is strange as the velocity contours do not show the curvature expected for rotation. The unusual velocity field in the northeast quadrant may be the result of $\mathrm{H} \mathrm{I}$ returning to the disk.

The two long, fairly symmetric, open tidal arms of NGC 5394 imply that the orbit of NGC 5395 was prograde and nearly in-plane with respect to NGC 5394. About this point, we refer the reader to the atlas of prograde, retrograde, and polar encounter simulations by Howard et al. (1993). The velocity of the companion relative to the disk material in prograde encounters is much smaller than in retrograde encounters; the resulting increase in the duration of strong tidal forces in prograde encounters leads to long, thin tidal arms. With a nearly in-plane orbit, the companion stays in the plane of the disk long enough to have a similar effect on the far side. The small difference in pitch angles between the two tidal arms of NGC 5394 indicates that the orbit of NGC 5395 had an initial tilt $\leq 30^{\circ}$ relative to the plane of NGC 5394 (see, for example, Howard et al. 1993).

NGC 5394 does not have the central eye-shaped structure visible in the ocular galaxies NGC 2535 and IC 2163. In the prograde encounter simulations by Elmegreen et al.
(1991) and Sundin (1993), the eye-shaped structure lasts for only a short time. Since NGC 5394 has had time to develop a starburst but its symmetric tidal arms are still prominent, it appears to be in a stage of post-encounter evolution a little past the transient ocular stage. The model in $\S 9$ below suggests that NGC 5394 is in an immediate post-ocular stage; the inner spiral arms develop from the ocular structure present at earlier times. It is possible that the inner spiral arms of NGC 5394 are density wave arms driven by the outer tidal arms. This would be similar to the situation in M51, where it appears that the outer tidal arms stimulate the corotation zone of the inner spiral pattern (Elmegreen, Elmegreen, \& Seiden 1989; Howard \& Byrd 1989). Although gas is present in the inner spiral arms of NGC 5394, star formation has not been triggered in the eastern inner arm. Because of the large velocity dispersion in the gas, the value of the instability parameter $Q_{\text {gas }}$ may be too high for significant star formation here unless the $\mathrm{H}_{2}$ surface density is large. We plan to make high-resolution ${ }^{12} \mathrm{CO}$ observations to see if most of the $3.5 \times 10^{9} M_{\odot}$ of molecular gas in the central ( $3.8 \mathrm{kpc}$ radius) region is concentrated in the nucleus, with little molecular gas at the location of the inner arms.

The two galaxies are rotating in opposite senses. NGC 5394 is being viewed nearly face-on, whereas NGC 5395 is being viewed at a considerable inclination, perhaps $i=65^{\circ}$. Because the encounter was prograde relative to NGC 5394, the encounter must have been retrograde at a considerable tilt angle relative to NGC 5395. This is qualitatively consistent with the major disturbed structures seen in NGC 5395. The fact that the northern $\mathrm{H}$ I tidal arm is not in the same plane as the disk of NGC 5395 implies that the orbit of NGC 5394 has a significant tilt relative to the disk of NGC 5395. As Howard et al. (1993) emphasize, tidal patterns

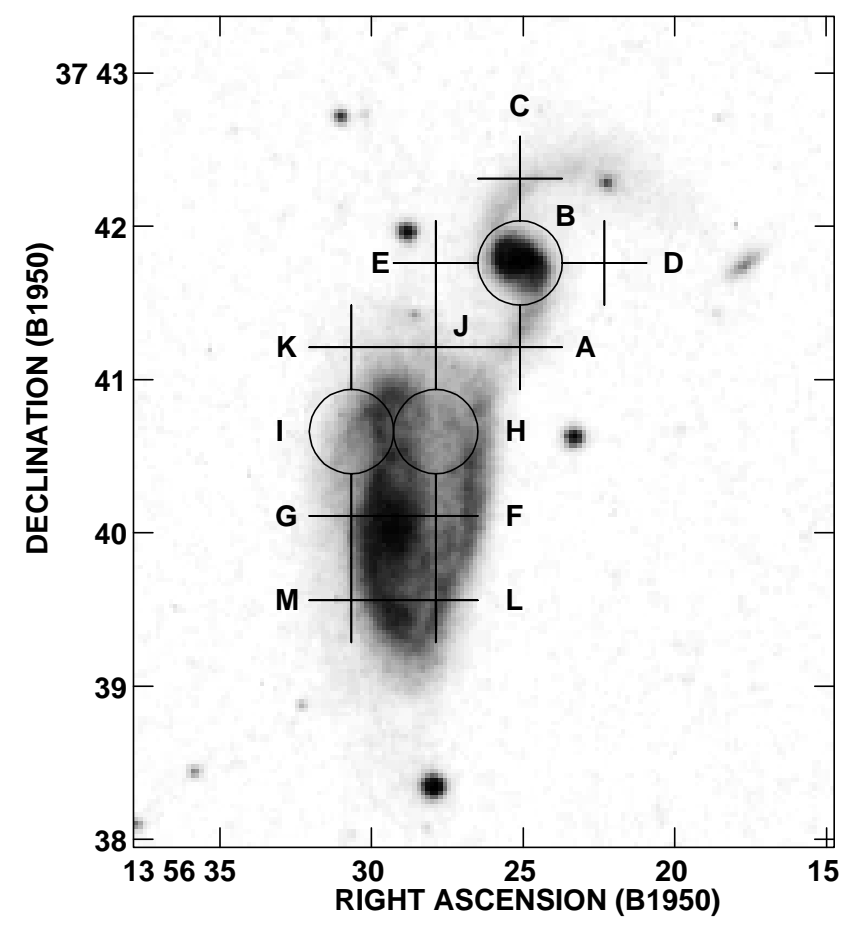

FIG. 27.- Gray-scale display of the Digitized Sky Survey image with symbols, equal in size to the ${ }^{12} \mathrm{CO}$ aperture, marking 13 positions where we searched for ${ }^{12} \mathrm{CO}(1-0)$ emission. The circles mark clear detections, and the plus signs mark the other positions. 
become more one-sided as the orbital tilt increases. Also, asymmetries in the velocity field and nonplanar disturbances are expected in noncentral collisions at high orbital tilt (see, for example, Appleton \& Struck-Marcell 1996).

Based on the $\mathrm{H}$ I velocity fields, the systemic velocity of NGC 5394 is $15 \pm 11 \mathrm{~km} \mathrm{~s}^{-1}$ greater than the systemic velocity of NGC 5395. This implies that the orbit of NGC 5394 is nearly in the sky plane. The constraint that the encounter be prograde relative to NGC 5394 then suggests the following orbit: travelling approximately in the plane of the sky, NGC 5394 could have crossed the disk plane of NGC 5395 south of the NGC 5395 nucleus, and then orbited counterclockwise to its present position northnorthwest of NGC 5395. Since the inclination of the disk of NGC 5395 is $65^{\circ}$, the orbit would be high inclination and retrograde relative to the disk of NGC 5395.

\section{ENCOUNTER SIMULATION}

The observations described in the previous sections constrain the nature of the collision in this system. Here we present a preliminary numerical model for the interaction computed with the star-gas smoothed particle hydrodynamics (SPH) code of Struck (1997). Since the code is described in detail in that paper, we give only a brief summary here. The SPH algorithm for the gas dynamics uses a spline kernel computed on a grid and a standard artificial viscosity formulation for modeling shocks. The halos of both galaxies in these models are represented by rigid, softened point-mass potentials. Local self-gravity is treated as explained in Struck (1997). The galaxy disks are initialized such that shear forces exceed self-gravity over larger scales. An adiabatic equation of state is used for the gas particles, with the addition of cooling terms and heating when the local gas density exceeds a fixed threshold density and star formation is assumed to occur.

The ratio of the companion mass to the primary mass is 0.25 , which is like that expected from the observations. To trace the structures that result from the encounter with adequate resolution, the model uses 13,640 gas particles and 4540 stars in the primary disk and 4940 gas particles and 4940 stars in the secondary disk. We used a large number of gas particles in the primary compared to the number of star particles so that the extensive $\mathrm{H}$ I structure can be discerned. Similarly, a large number of stars is used in the companion to make the long tidal structures discernible. Since this is not a fully self-consistent, $N$-body code, the relative numbers of star and gas particles in the primary and secondary do not affect the simulation dynamics. In both model galaxies the initial gas disk is larger than the stellar disk by a factor of 1.8 in the primary and 1.2 in the companion. The primary gas disk is also initially 1.8 times as large as the companion gas disk.

The simulation code is dimensionless, but we adopt a representative set of units. To approximate the observed long-rising rotation curve of the primary disk, we set the scale length of the halo potential as $8 \mathrm{kpc}$ and the scale velocity, defined as the circular orbit velocity at a radius of one scale length, as $190 \mathrm{~km} \mathrm{~s}^{-1}$. The time unit $\left(4.2 \times 10^{7} \mathrm{yr}\right)$ is the time to move one radian at the circular velocity of 190 $\mathrm{km} \mathrm{s}^{-1}$ and a radius of $8 \mathrm{kpc}$.

Figure 28 shows two orthogonal views of the model at a time $t_{1}=1.7 \times 10^{7} \mathrm{yr}$ after closest approach, when the model appears to resemble most closely the observed system. The $x-y$ plane is approximately the plane of the sky, with the $z$-direction headed toward us (it is a right-handed coordinate system) and the $y$-coordinate roughly along the declination axis. The $x-y$ plane was the initial plane of the companion disk, and, as suggested by the observations, the primary was initially rotated out of this plane by $60^{\circ}$ around the $y$-axis. The bottom panel ( $x-z$ plane) shows that the primary is still centered on its initial plane at this time, albeit with distortions into the third dimension. On the eastern side of the primary, gas structures in the outer part occur both in front of and behind the plane defined by the inner stellar disk. The companion disk is tilted by about $20^{\circ}$ from its initial plane and, in fact, seems to lie close to its orbital plane. However, this small tilt is not detectable in the $x-y$ plane (top panel), since the internal spiral waves elongate the disk in the direction of the foreshortening.

Figure 28 also shows the orbit of the companion relative to the (fixed) primary. This is a bound orbit, and the companion is started at the point marked by an open circle with a modest velocity toward the primary. The plotted trajectory is continued somewhat beyond the best-fit time $t_{1}$. The initial position and velocity of the companion are rather arbitrary within the constraints that the collision must be retrograde for the primary, prograde for the companion, and the orbital inclination is relatively high at impact.

Figure 29 shows the stellar and gas components from Figure 28 separately, with an enlarged view of the stellar component.

The model reproduces qualitatively many of the observed features of the small galaxy, NGC 5394. In the model, as well as in the observations, NGC 5394 has a long curved tidal tail on the side opposite the primary galaxy, a tidal bridge arm that points toward the primary (see bottom panel of Fig. 29), and broad inner stellar arms, with a discontinuity between the internal spiral arms and the outer tidal arms. As expected from the work by Elmegreen et al. (1991), the inner spiral arms developed from a strong ocular wave visible at earlier times. The inner part of the disk in the secondary is largely composed of material that lost angular momentum in the collision and moved inward. This is consistent with the observation of a starburst in NGC 5394.

The observations and model of NGC 5394 differ in several respects as well. In the model, the tidal tail of the secondary is considerably longer than the observed tidal tail of NGC 5394. The model tidal tail is also well populated throughout by gas and stars (the gas is displaced to the concave side of the stellar arm), whereas in NGC 5394, H I gas with $N(\mathrm{H} \mathrm{I}) \geq 2 \times 10^{20}$ atoms $\mathrm{cm}^{-2}$ is detected only along the inner third of the arc length of the stellar tidal tail. In the model, the tidal bridge from the secondary is less well organized than the tidal tail, whereas in NGC 5394 the tidal tail and bridge look symmetric and have about the same stellar arm-interarm contrasts out to $40^{\prime \prime}$ from the nucleus (see Fig. 7). The faintness of the bridge in the model results primarily because the particles are stretched over a large separation between the two galaxies; this arises because the numerical algorithm does not include dynamical friction. In the model, the location of the secondary is somewhat east of the observed location of NGC 5394 and farther from the primary.

The morphology of the primary, NGC 5395 is more complex. The prominent arm I on the western side of the galaxy (see Figs. 1 and 12 ) is clearly present in the top panels of Figures 28 and 29. In the models it is visible in the 

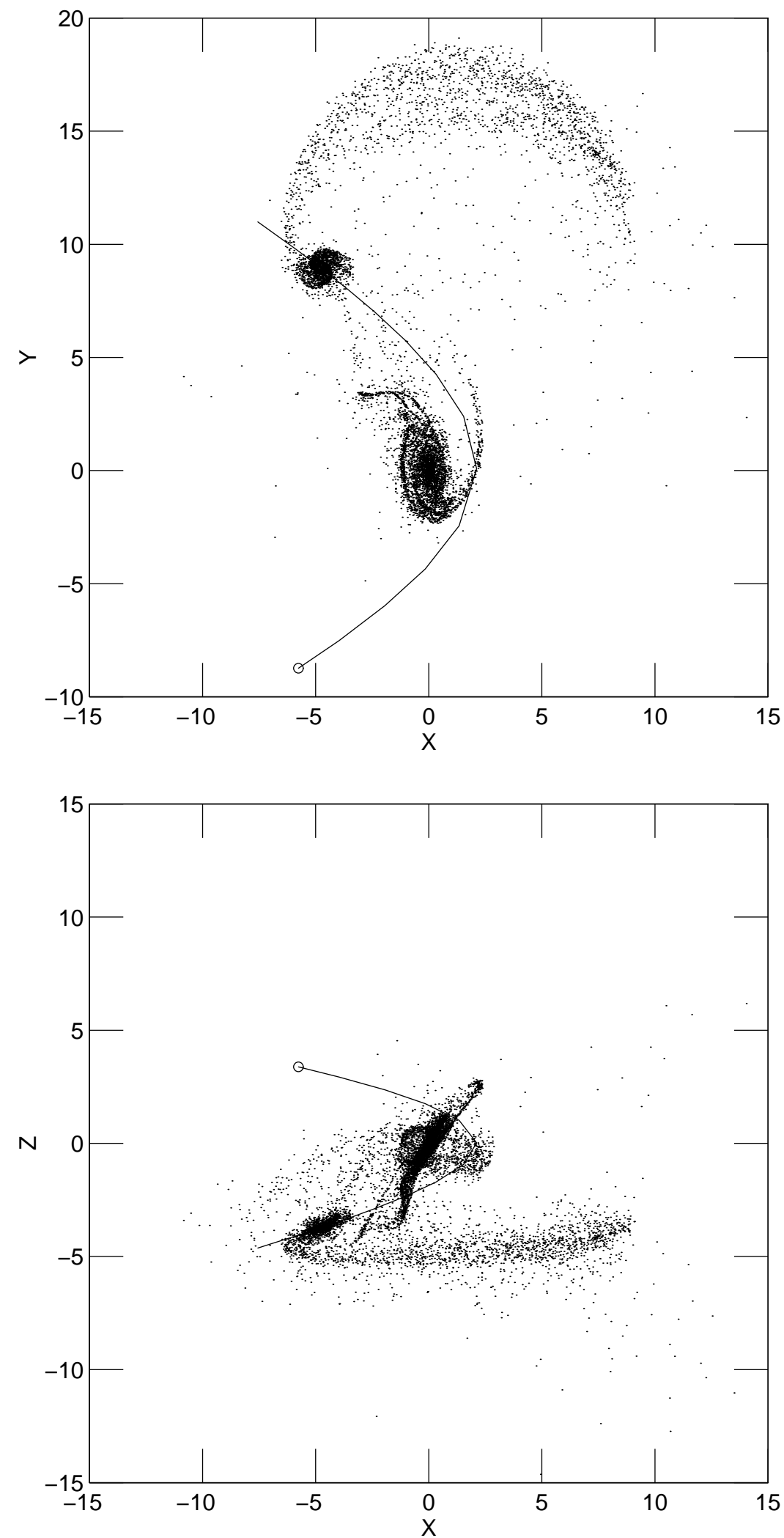

FIG. 28.-Two orthogonal views of the combined gas and star particle distributions at a time when the numerical model most closely matches the observations (time $\left.t_{1}\right)$. The units are computer length units $(1 \mathrm{clu}=8 \mathrm{kpc})$. Stars originating in the companion but accreted onto the primary are eliminated by plotting only those companion stars with coordinate value $y>3.0$. The trajectory of the center of the companion galaxy is drawn in a coordinate system in which the primary center is fixed (see text). The companion was started at the position of the open circle. The positive $z$-axis points in the approximate direction of the observer, and the positive $y$-axis corresponds roughly to the declination axis. 

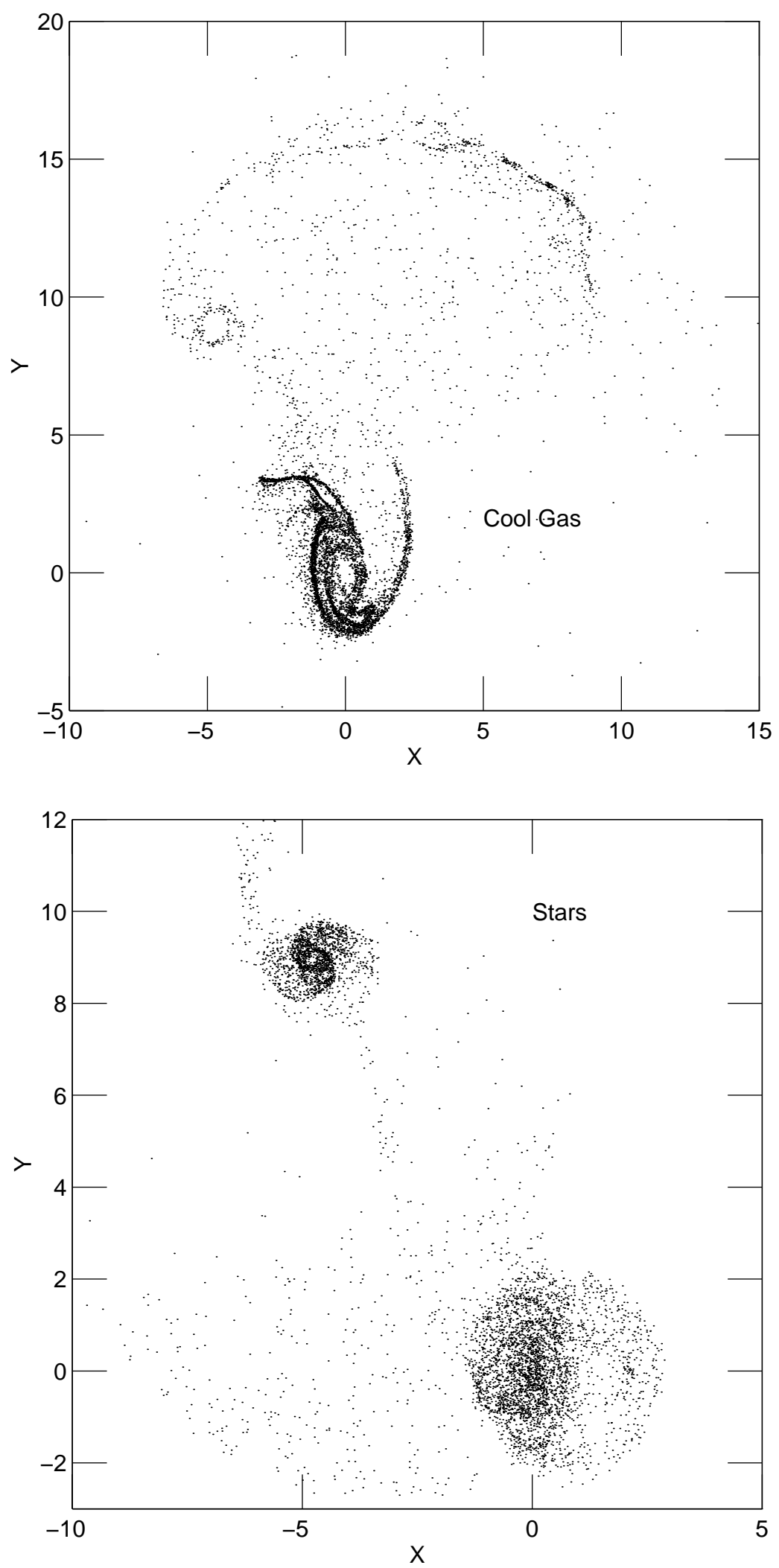

Fig. 29.- - Slightly enlarged views of different components of the system at time $t_{1}$ in the $x-y$ plane of Fig. 28. The units are computer length units $(1$ $c l u=8 \mathrm{kpc}$ ). Top: The gas particle distribution, with half of the particles with temperatures less than 5 times the fiducial temperature plotted (i.e., those with temperatures of less than about $2.5 \times 10^{4} \mathrm{~K}$ in the scaling of the text). Bottom: Stellar distribution, with half the stars plotted.

gas, but not the old stars, because the initial gas disk is much larger than the stellar disk. This arm is also much more open in the models than in the observations. The bottom panel of Figure 28 shows that the western arm is tilted relative to the mean plane of the disk of NGC 5395, so its openness is in part a function of viewing angle. However, it is also a function of the impact parameter of the collision, the potential in the outer disk, and time. (It is less open at 
earlier times in this model.) Varying these parameters to optimize the fit should further constrain the model.

The top panels of Figures 28 and 29 also show that there is a good deal of gas in the northeast part of the model primary, in agreement with the observations (see Fig. 13). In the model most of this material is in a spiral spur, which might be related to the northern $\mathrm{H}$ I tidal arm of Figure 16 or, possibly, to the north-northeast extension. If it is related to the northern tidal arm, then the model suggests that this tidal arm is not directly connected to the western arm, but to material at slightly smaller radii. We also note the large gap running down the middle of this spur; this seems to be kinematic caustic in the warped disk. The gap appears sheared and stretched in the figures, but it was rounder at earlier times. Perhaps it is the observed caustic 1 . In the model, only a small number of gas particles are scattered to the south and west of the primary, where the observations detect the broad, diffuse, southern $\mathrm{H}$ I tidal arm. This suggests that the initial disk may have had more gas outside of the impact radius than assumed in the model, or that the observed feature may be the result of a prior encounter.

The top panels of Figures 28 and 29 show that the western arm of the primary connects inward to a spiral that winds around the southern part of the primary to the east side and probably continues around the north as well in a clockwise direction, before connecting with an inner ringlike structure. Previous time steps show that this is an expanding ring wave. (In the initial disk, particles extend nearly to the center of the galaxy.) Thus, in the model, the primary has both a collisional ring wave and a mostly material western arm that is pulled away from the disk plane because of the perpendicular flyby. The inner ring also has a very strong spiral connection to the outer spiral in the southeast and south. Except for this last feature, these spirals fit the optical image of NGC 5395.

There is one set of features in the models that is completely absent in the observations: a very large-scale shell and polar-ring disk formed from stars ripped off the companion at closest approach. These features have been removed from the top panel of Figure 28, but are clear in the bottom panels of Figures 28 and 29; i.e., the large-scale shell is the scattering of particles east of the primary out to $x=-10$ and the polar-ring disk is the feature nearly perpendicular to the disk of the primary in the $x-z$ plane. Whereas the shell may have too low a surface brightness to be detected, it seems less likely that the inner, polar disk would also be invisible. Perhaps the collisional torque on the companion is too large in this model, although a large torque is needed to get the large tidal tails and compressed disk of the companion. We plan to undertake a more extensive modeling program to try to resolve such difficulties.

Finally, Figure 30 displays the line-of-sight velocity versus the $y$ coordinate analogous to the velocitydeclination diagram of Figure 19 that is summed over R.A. Positive velocity in Figure 30 is away from us, to agree with the usual convention. The general agreement between the structures in this figure and those of Figure 19 and the specific components in Figure 14 provides additional assurance of the basic correctness of the model. In the model, as in the observations, $\left(v-v_{\mathrm{sys}}\right)$ is less negative on the tidal arm of the primary than in the disk at the same projected location, so in Figure 30 the tidal arm of the primary and the bridge material lie between the disk of the primary and the disk of the companion, as in Figure 19. The only major

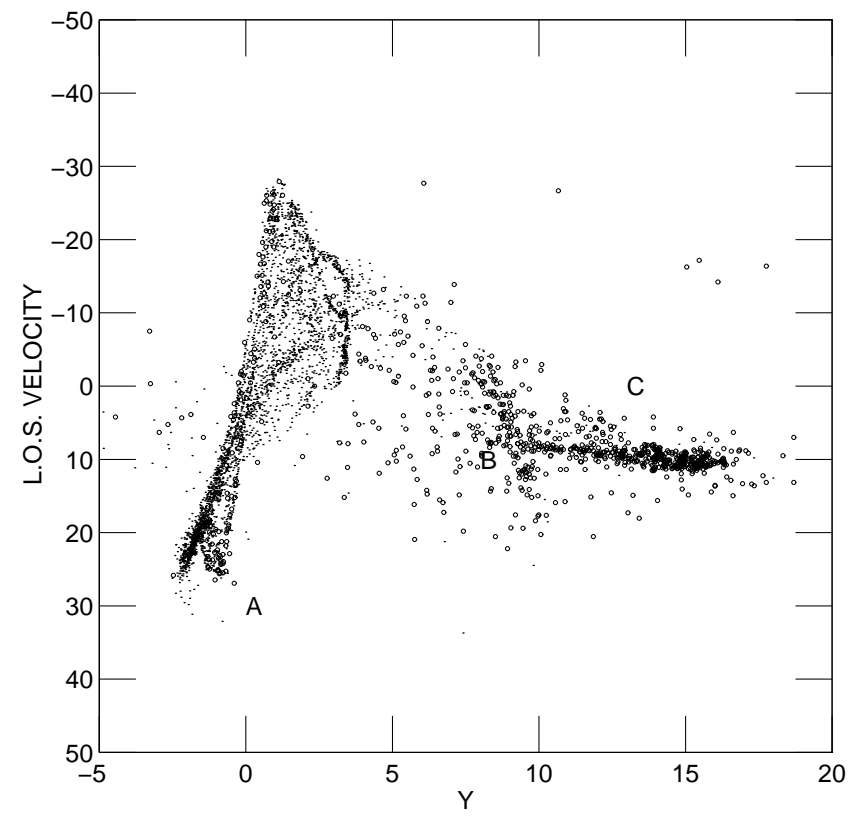

FIG. 30.- Simulational velocity-longitude diagram (analogous to Fig. 19), showing the line-of-sight velocity component vs. the $y$-coordinate of the model at the same time as the previous figures. The agreement with the observed P-V diagram is good if we take the velocity unit as $9 \mathrm{~km} \mathrm{~s}^{-1}$. The model $y$-coordinate is similar to the declination coordinate used in the observational velocity-longitude diagrams, and positive velocity is away from the observer. Half of the cool gas particles are plotted, as in the previous figure. The open circles indicate particles originating in the companion, dots are those from the primary. The vertical feature marked with the letter " $\mathrm{A}$ " is the disk of the primary, and the disk of the companion is marked with the letter "B." The tidal arm and bridge material lie between them as in Fig. 20. There is no detected $\mathrm{H} \mathrm{I}$ emission that corresponds to the feature marked " $\mathrm{C}$," which consists of particles in the long tidal tail of the companion.

disagreements are feature $\mathrm{C}$ (the gaseous tidal-tail of the companion) and the lack of a broad, diffuse southern $\mathrm{H} \mathrm{I}$ tidal arm in the model.

\section{COMPARISON OF NGC 5394 WITH TWO OCULAR GALAXIES}

We compare the properties of the post-ocular galaxy NGC 5394 with those of the ocular galaxies IC 2163 (Elmegreen et al. 1995a; Elmegreen et al. 1995b) and NGC 2535 (Kaufman et al. 1997), studied previously, to see the evolution of structures resulting from prograde, in-plane encounters. All three galaxies have intrinsically oval disks, long tidal arms with a stellar arm-interarm contrast that is large compared to normal density wave arms, and widespread high-velocity dispersion in the H I gas. IC 2163 is the least evolved of the three. It has a pronounced eye-shaped oval whose rim is outlined by $\mathrm{H} \mathrm{I}$ and radio continuum emission, a double parallel arm structure (tidal tail plus streaming arm) on its anticompanion side, streaming motions around the eye-shaped oval, and massive $\left(10^{8} M_{\odot}\right)$ $\mathrm{H}$ I clouds on the tidal arms. NGC 2535 is in a slightly later stage of post-encounter evolution than IC 2163. NGC 2535 has an eye-shaped oval with streaming motions around the oval, very bright $\mathrm{H}$ in regions along the southern rim of the oval and at the two apices of the oval, and massive $\mathrm{H} \mathrm{I}$ clouds on the tidal arms. The rim of the oval is not outlined by $\mathrm{H}$ I and radio continuum emission, and the two components of the tidal tail have already merged. There is evi- 
dence of mass transfer from NGC 2535 to its small starburst companion. NGC 5394 is in a still later stage of postencounter evolution. The eye-shaped oval has evolved into inner spiral arms (only one of which is detected in $\mathrm{H} \alpha$ ), and most of the gas has fallen into the central region, where it fuels a starburst. There are no massive $\mathrm{H}$ I clouds on the tidal arms of NGC 5394; a high-velocity $6 \times 10^{7} M_{\odot}$ cloud found in the direction of NGC 5394 is probably tidal debris from itself or from its companion.

\section{CONCLUSIONS}

$\mathrm{H}$ I, radio continuum, $\mathrm{H} \alpha$, broadband optical, nearinfrared and ${ }^{12} \mathrm{CO}$ observations allow us to see the effect of the encounter on the motions and distribution of the gas and stars in the galaxy pair NGC 5394/95. NGC 5394 is in an immediate post-ocular phase, with a central starburst, an intrinsically oval disk, two long, open tidal arms with high arm-interarm contrast, and very bright inner spiral arms disjoint from the outer tidal arms. Most of the gas in NGC 5394 is in molecular form and concentrated within $3.8 \mathrm{kpc}$ of the center and so is suitable for fueling the starburst. Despite the presence of $\mathrm{H}$ I gas, two of the three optically bright inner spiral arms of NGC 5394 show no evidence of ongoing star formation. If molecular gas is ignored, then the instability parameter $Q_{\text {gas }}$ is too high for significant star formation at these two inner arms. Higher resolution ${ }^{12} \mathrm{CO}$ observations are needed to see how much molecular gas is located at the inner arms.

In the large companion, NGC 5395, we find a large ring or pseudo-ring of $\mathrm{H} \alpha$, radio continuum, and $\mathrm{H}$ I emission (somewhat off-center with respect to the nucleus), noncircular motions, a large stellar caustic feature, a long narrow $\mathrm{H}$ I tidal in the north, and a broad diffuse $\mathrm{H}$ I tidal arm in the south. The H I trough in the center of NGC 5395 is not filled in with ${ }^{12} \mathrm{CO}$ emission; thus the ring is a ring in total gas as well as in $\mathrm{H} \mathrm{I}$. The eastern side of the ring is brighter in radio continuum and $\mathrm{H} \alpha$; the western side is brighter in $\mathrm{H}$ I and contains massive $\left(10^{8} M_{\odot}\right) \mathrm{H}$ I clouds not associated with the most luminous $\mathrm{H}$ iI regions. The interaction has produced nonplanar structures in NGC 5395. From the $\mathrm{H}$ I velocity data, we find that the northern tidal arm and a broad extended feature north-northeast of the ring are not in the same plane as the disk. Optically, there is evidence for nonplanar structures on the western side of the galaxy. The $\mathrm{H}$ I velocity field throughout the northeast quadrant of NGC 5395 is strange; this may result from the disk in this quadrant folding toward us and/or from material returning to the disk.

Using a collision that is prograde relative to NGC 5394 and retrograde at a high tilt angle relative to the disk of NGC 5395, a preliminary numerical model for the encounter accounts for a number of the observed features. For NGC 5394, the model successfully reproduces the long tidal tail, the tidal bridge arm, the inner spiral arms, and the intrinsically oval shape to the disk. In particular, the model finds that the inner spiral structure of NGC 5394 developed from the ocular structure at slightly earlier times. For NGC 5395, the model successfully reproduces the ring/spiral structure that includes the western arm, the northern $\mathrm{H} \mathrm{I}$ tidal arm, the relative locations in the position-velocity diagram of the main disk and the nonplanar structures north of the ring, and a kinematic caustic that may relate to the observed caustic feature.

NGC 5394 and the two ocular galaxies (IC 2163 and NGC 2535) studied by Elmegreen et al. $(1995 \mathrm{a} ; 1995 \mathrm{~b})$ and Kaufman et al. (1997), respectively, provide snapshots of two stages of the development of the ocular morphology and one of the immediate post-ocular stage. The comparison of models and observations of these systems confirms the collisional theory of how this morphology forms in prograde interactions. The present system extends our understanding of collisional ring galaxies as well as oculars. Specifically, the agreement between the preliminary numerical model and the wave structure in NGC 5395 allows us to infer that the collision which generated these waves had a relatively large impact parameter and an important retrograde aspect to the orbit of the companion. In fact, compared to other collisional ring galaxies studied thus far (e.g., see the review in Appleton \& Struck 1996), the encounter that produced the ring/spiral structure of NGC 5395 had one of the largest impact radii and one of the most retrograde orbits.

We thank Beverly Smith for helpful conversations about the models. For the Fick Observatory images, we thank Joe Eitter and Philip Appleton. We thank Tyler Nordgren for providing the fits version of his $r$-band image. This research was partially supported by a Fullam-Dudley award to M. Kaufman and by a grant to M. Kaufman from NASA administered by the American Astronomical Society.

\section{REFERENCES}

Appleton, P. N., \& Struck-Marcell, C. 1996, Fundam. Cosmic Phys., 16, 111

Arp, H. 1969, A\&A, 3, 418

Baars, J. W. M., Genzel, R., Pauliny-Toth, I. I. K., \& Witzel, A. 1977, A\&A, 61,99

Becker, R. H., White, R. L., \& Helfand, D. J. 1995, ApJ, 450, 559

Bohlin, R. C., Savage, B. D., \& Drake, J. F. 1978, ApJ, 224, 132

Condon, J. J., Helou, G., Sanders, D. B., \& Soifer, B. T. 1990, ApJS, 73, 359

de Vaucouleurs, G., de Vaucouleurs, A., Corwin, H. G., Jr., Buta, R. J., Paturel, G., \& Fouqué, P. 1991, Third Reference Catalogue of Bright Galaxies (New York: Springer)

Elmegreen, B. G., Elmegreen, D. M., \& Seiden, P. E. 1989, ApJ, 343, 602

Elmegreen, B. G., Kaufman, M., \& Thomasson, M. 1993, ApJ, 412, 90

Elmegreen, B. G., Sundin, M., Kaufman, M., Brinks, E., \& Elmegreen, D. M. 1995 b, ApJ, 453, 139

Elmegreen, D. M., \& Elmegreen, B. G. 1984, ApJS, 54, 127

Elmegreen, D. M., Elmegreen, B. G., Chromey, F. R., Hasselbacher, D. A., \& Bissell, B. A. 1996, AJ, 111, 1880

Elmegreen, D. M., Kaufman, M., Brinks, E., Elmegreen, B. G., \& Sundin, M. 1995a, ApJ, 453, 100

Elmegreen, D. M., Sundin, M., Elmegreen, B. G., \& Sundelius, B. 1991, A\&A, 244, 53

Hibbard, J. E. 1995, Ph. D. thesis, Columbia Univ.

Hibbard, J. E., \& Mihos, J. H. 1996a, AJ, 110, 140

Hibbard, J. E., \& van Gorkom J. H. 1996b, AJ, 111, 655

Howard, S., \& Byrd, G. G. 1989, AJ, 99, 1798

Howard, S., Keel, W. C., Byrd, G. G., \& Burkey, J. 1993, ApJ, 417, 502

Irwin, J. A. 1994, ApJ, 429, 618

Irwin, J. A., \& Seaquist, E. R. 1991, ApJ, 371, 111

Jogee, S., Kenney, J. D. P., \& Smith, B. J. 1998, ApJ, 494, L185

Karachentsev, I. D. 1980, ApJS, 44, 137

Kaufman, M., Brinks, E., Elmegreen, D. M., Thomasson, M., Elmegreen, B. G., Struck, C., \& Klarić, M. 1997, AJ, 114, 2323

Keel, W. C., Kennicutt, R. C., Jr., Hummel, E., \& van der Hulst, J. M. 1985, AJ, 90, 708

Kennicutt, R. C., Jr. 1989, ApJ, 344, 685

Klarić, M. 1993, Ph. D. thesis, Univ. Alabama

Kodeira, K., Okamura, S., \& Ichikawa, S. 1990, Photometric Atlas of Northern Bright Galaxies (Tokyo: Univ. of Tokyo Press)

Krumm, N., \& Salpeter, E. E. 1980, AJ, 85, 1312

Mihalas, D., \& Binney, J. J. 1981, Galactic Astronomy (San Francisco: Freeman)

Nordgren, T. E., Chengalur, J. N., Salpeter, E. E., \& Terzian, Y. 1997, AJ, 114,77

Persic, M., \& Salucci, P. 1991, ApJ, 368, 60

Regan, M., \& Elmegreen, D. M. 1997, AJ, 114, 965 
Roberts, W. W., \& Hausman, M. A. 1984, ApJ, 277, 744

Schoenmakers, R. H. M., \& Swaters, R. A. 1999, in ASP Conf. Ser. 182, Galaxy Dynamics, ed. D. Merritt, J. A. Sellwood, \& M. Valluri (Provo: ASP), 277

Sharp, N. A., \& Keel, W. C. 1985, AJ, 90, 469

Smith, B. J. 1994, AJ, 107, 1695

Strong, A. W., Bloemen, J. B. M., Dame, T. M., Grenier, I. A., Hermsen, W., Lebrun, F., Nyman, L.-A., Pollock, A. M. T., \& Thaddeus, P. 1998, A\&A, 207, 1

Struck, C. 1997, ApJS, 113, 266

Sundelius, B., Thomasson, M., Valtonen, M. J., \& Byrd, G. G. 1987, A\&A, 174,67
Sundin, M. 1993, Ph.D. thesis, Univ. Göteborg and Chalmers Univ. of Technol.

Tenorio-Tagle, G. 1981, A\&A, 94, 338

Toomre, A. \& Toomre, J. 1972, ApJ, 178, 623

Van der Hulst, J. M., Kennicutt, R. C., Crane, P. C., \& Rots, A. H. 1988, A\&A, 195, 38

Visser, H. C.D. 1980a, A\&A, 88, 149

1980b, A\&A, 88, 159

Wray, J. D. 1988, The Color Atlas of Galaxies (Cambridge: Cambridge Univ. Press) 